SERVIÇO DE PÓS-GRADUAÇÃO DO ICMC-USP

Data de Depósito: 20.01.2005

Assinatura: fitne Taue lambon juena

\title{
Formas normais para equações diferenciais funcionais
}

Rodrigo da Silva Rodrigues

Orientadora: Profa. Dra. Sueli Mieko Tanaka Aki

Dissertação apresentada ao Instituto de Ciências Matemáticas e de Computação - ICMC-USP, como parte dos requisitos para obtenção do título de Mestre em Matemática.

USP - São Carlos

Janeiro/2005 
Aluno: Rodrigo da Silva Rodrigues

\section{A Comissão Julgadora:}

Profa. Dra. Sueli Mieko Tanaka Aki

Prof. Dr. Eduardo Alex Hernandez Morales

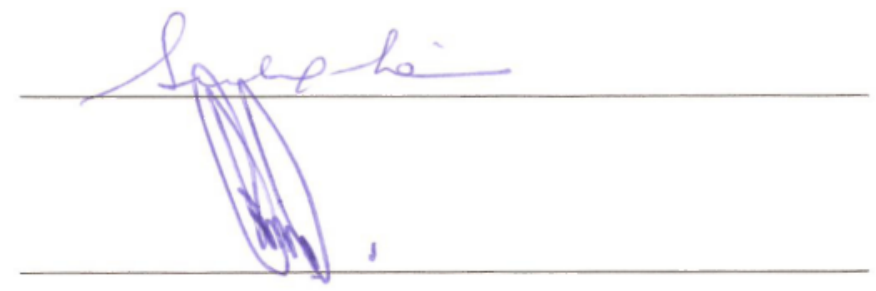

Profa. Dra. Maria Aparecida Bená

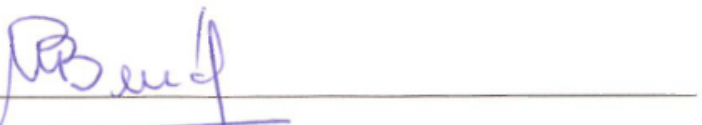


"Grandes realizaçōes não são foitas por inpulso, nitas por uma soma de pequenas rcalizaços."

(Vincent Van Gogh) 
Aos mens pais, Carmo Rodrigues e Maria de Lourdes Aos meus irmãos,

Leandro e Milene E à minha esposa Taciana dedico. 


\section{Agradecimentos}

Ao concluir este traballo agradego:

À Dens.

Aos uneus pais e irmäos, que com anor, me apoiam e sempre estão presentes em lodos os momentos de minla vida.

A miuha família, avós, tios e primos pelo grande incentivo.

$\grave{A}$ minhla esposa Taciana pelo sen anor, anizade eseu conhecimento

$\Lambda$ banca cxaminadora.

A Prof. Dra. Sueli Mieko Tanaka Aki, pela amizade, paciência e orientaç̧ào.

Aos professores do Departanento de Matemática da FCT - UNESP (Campus de Presidente Prudente) pela amizade e pelo conlecimento transmitido. En especial ao Prof. Dr. José Carlos Rodrignes, pela paciència e amizade sempre presentes desde os tempos da graduaçàs).

$\grave{A}$ Angela, Everaldo, Mariene o Anderson pela amizade e apoio que sempre se fez presente.

Aos colegas da P’ós-Graduação, pelo agradável convívio.

Aos professores do Departamento de Matemática do ICMC - USP polo contrecimento transmitide.

A CAl'ES pelo apoio financeiro. 


\section{Resumo}

Este traballosé dedicado à extensão do Método da Forma Normal para Equaçóes Diferenciais Ordinárias às Equaçoes Diferenciais Funcionais Retardadas. O mótodo da forma normal para equaçòes diferenciais funcionais retardadas nos dará o fluxo sobre una variedade localmente invariante de dimensão finita através de uma equação diferencial ordinária. Como aplicaçào, calcularemos a forma normal para equação diferencial funcional relardada escalar com uma singularidade do tipo Bogdanov-Takens. Analisaremos também a forma normal para equações diferenciais funcionais retardadas com parâmetro. Finalizaremos este trabalho com o cálculo da forma normal de um sistema planar com singularidade do tipo Bogdanov-Takens.

Palavras-Chave: Formas Normais, Equaçoes Diferenciais Ordinárias, Equaçoes Diferenciais Funcionais Retardadas, Singularidade do Tipo Bogdanov-Takens. 


\section{Abstract}

In this work, we compute the normal forms associated with the flow on a finitedimensional invariant manifold tangent to an invariant space for the infinitesimal generafor of the linearized equation at the singularity. As an application, the Bogdanov-'Takens singularity is considered.

Keywords: Normal Forms, Ordinary Differential Equations, Retarded Functional Differential Equations, Bogdanov-'Takens Singularity. 


\section{Sumário}

1 Preliminares

1.1 Introdnção . . . . . . . . . . . . . . . . . . . 9

1.2 Equaçoes Diferenciais Funcionais Retardadas . . . . . . . . . . . . . 9

1.3 Semigrupos para Equações Lineares Autonômas . . . . . . . . . . . . . . 15

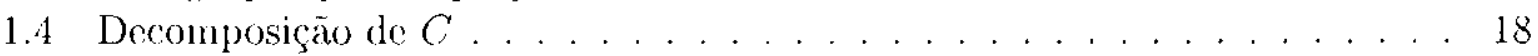

1.5 Decomposição de $C$ com a Equação Adjunta . . . . . . . . . . . . . . 21

1.6 Teorema das Variedades Invariantes . . . . . . . . . . . . . 23

2 Formas Normais para Equações Diferenciais Ordinárias $\quad 27$

2.1 Introdução . . . . . . . . . . . . . . . . . . . . . . . 27

2.2 Formas Normais para lénaçoes Diferenciais Ordinárias . . . . . . . . . 27

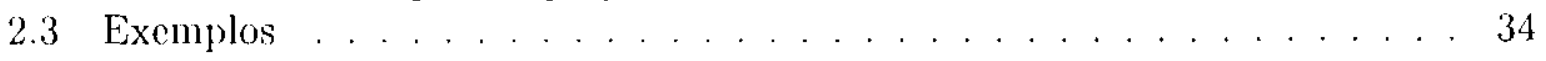

3 Formas Normais para Equaçōes Diferenciais Funcionais 37

3.1 Introdução . . . . . . . . . . . . . . . . . . . . . . . 37

3.2 Equacoes Diferenciais Funcionais . . . . . . . . . . . . 37

3.3 Formas Normais . . . . . . . . . . . . . . . . . . . . 41

3.4 Condições de Não-Ressonância . . . . . . . . . . . . . . . . 46

3.5 Equacões Diferenciais Funcionais con Parâmetro . . . . . . . . . . . 58

3.6 Formas Normais . . . . . . . . . . . . . . . . . . . . . . . . . 60

3.7 Singularidade do tipo Bogdanov-Takens . . . . . . . . . . . . . . . 62

3.8 Singularidade do tipo Bogdanov-Takens com um parâmetro . . . . . . . 81

4 Sistema Planar com Dois Retardos $\quad \mathbf{8 5}$

4.1 Introdução . . . . . . . . . . . . . . . . . . . 85

4.2 O Problema de Autovalor . . . . . . . . . . . . . . . 85

4.3 Singmlaridade de Bogdanov-Takens . . . . . . . . . . . 95

$\begin{array}{ll}\text { Referências Bibliográficas } & 109\end{array}$ 


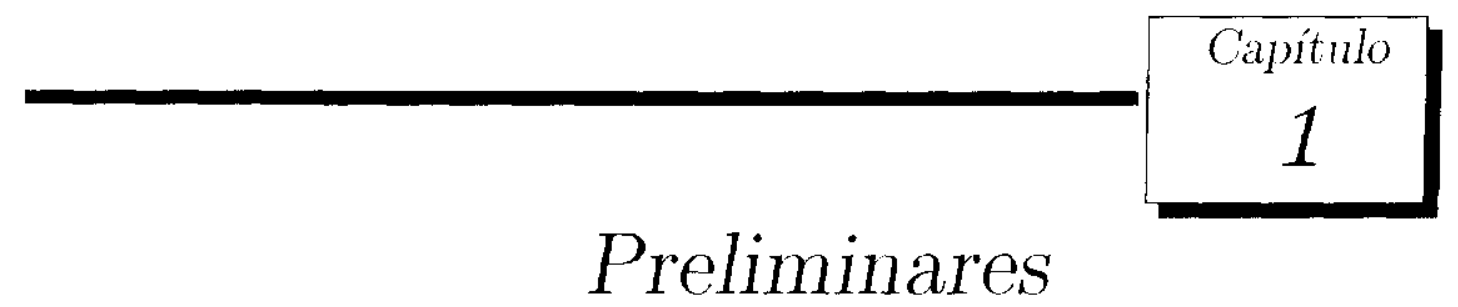

\subsection{Introdução}

Neste capítulo, apresentaremos alguns conceitos e resultados que serào fundamentais no decorrer deste trabalho.

Iniciaremos formalizando o conceito de equações diferenciais funcionais retardadas e apresentaremos o teorema de existência e unicidade de soluções para esse tipo de equação. Faremos uma breve introdução à teoria de semigrupos para equações diferenciais funrionais lineares autônomas. Nas Seçós 1.4 c 1.5, vamos decompor o espaço das funções contínuas de $[-r, 0]$ sobre $\mathbb{R}^{n}$ en soma direta de um espaço de dimensão finita $P$ com IIn espaço $Q$, ambos invariantes pelo gerador infinitesimal $A_{0}$. Introduziremos a forma bilinear a qual nos permitirá definir explicitamente o espaço $Q$ e determinaremos unia projeção de $C$ sobre os autoespaços gencralizados.

Finalizaremos este capítulo com o teorema das variedades invariantes, o qual analisa sua existência para equaçōes diferenciais funcionais retardadas autônomas. Esse teorema cxamina o comportamente de solução próximas de un ponto de equilíbrio de uma equação diferencial funcional retardada antônoma e estuda localmente a cxistência de variedades estáveis, instáveis, centrais,...

\subsection{Equações Diferenciais Funcionais Retardadas}

Nesta seção, definiremos equações diferenciais funcionais retardadas. Mas. antes de darmos a definição formal desse conceito, consideraremos un caso mais simples de equação diferencial retardada. Finalizaremos esta seção demonstrando a existência e unicidade de soluçōes para lais equaçòes.

Consideremos a equação diferencial retardada:

$$
\dot{x}=f(t, x(t-r)) \text {, }
$$

onde $r$ com real nào negativo, $x$ denota a derivada de $x$ em relaçio a $t, f:[0, \infty) \times \mathbb{R} \longrightarrow \mathbb{R}$ c $x: I \subset \mathbb{R} \longrightarrow \mathbb{R}$ são contínuas para $t \geq 0$.

Analisaremos a equação diferencial retardada (1.1), para $r>0$. Como sabemos, para obtermos a solução de uma exuação diferencial ordinária, é preciso conhecer o valor da solução en um instante $t_{0}, x_{0}=x\left(t_{0}\right), x_{0}$ é dita condição inicial. Mas, para a equação diferencial retardada (1.1), precisamos conhecer a solução em um intervalo antcrior ao 
ponto $t_{0}$. Eun outras palavras, precisamos conhecer $11 n$ 'certo' passado da solução anterior ao ponto to.

Suponlamos $x:[-r, \infty) \longrightarrow \mathbb{R}$, c consideremos o seguinte sistema:

$$
\left\{\begin{array}{l}
\dot{x}(t)=f(t, x(t-r)), \text { para } t \geq 0, \\
x(\theta)=x_{0}(\theta), \text { para } \theta \in[-r, 0],
\end{array}\right.
$$

onde $x_{0}(\theta)$ é contínua em $[-r, 0]$.

Denotaudo por $x_{1}(t)$, a solução do problema (1.2) sobre o intervalo $[0, r]$, temos que $x_{1}$ satisfaz:

$$
\dot{r}_{1}(t)=f\left(t, x_{1}(t-r)\right)
$$

Mas, para $t \in[0, r]$, segue que $t-r$ pertence à $[-r, 0]$, e portanto, obtemos:

$$
x_{1}(t-r)=x_{0}(t-r) \text {, para } t \in[0, r] .
$$

Logo, conseguimos:

$$
\left\{\begin{array}{l}
\dot{x}_{1}(t)=f\left(t, x_{0}(t-r)\right) \\
x_{1}(0)=x_{0}(0) .
\end{array}\right.
$$

Integrando a primeira equação do sistema acima, temos:

$$
\int_{0}^{t} \dot{x}_{1}(\tau) d \tau=\int_{0}^{t} f\left(\tau, x_{0}(\tau-r)\right) d \tau, \quad t \in[0, r]
$$

centào,

$$
x_{1}(t)-x(0)=\int_{0}^{t} f\left(\tau, x_{0}(\tau-r)\right) d \tau, \quad t \in[0, r]
$$

()11 seja.

$$
x_{1}(t)=x_{0}(0)+\int_{0}^{t} f\left(\tau, x_{0}(\tau-r)\right) d \tau, \quad t \in[0, r] .
$$

Analogamente, para $t \in[r, 2 r]$, denotemos a solução por $x_{2}$.

Logo,

$$
\dot{x}_{2}(t)=f\left(t, x_{2}(t-r)\right) .
$$

Mas, quando $t \in[r, 2 r]$, temos que $t-r$ pertence à $[0, r]$, e portanto,

$$
x_{2}(t-r)=x_{1}(t-r)
$$

Assinn, obtomos:

$$
\left\{\begin{array}{l}
x_{2}(t)=f\left(t, x_{1}(t-r)\right) \\
x_{2}(r)=x_{1}(r)
\end{array}\right.
$$

Donde, conseguinos:

$$
x_{2}(t)=x_{1}(r)+\int_{r}^{t} f\left(\tau, x_{1}(\tau-r)\right) d \tau,
$$


(a)tit $t \in[r, 2 r]$.

Conchundo, para todo $t \geq 0$, momoms $n \in \mathbb{N}$ com $t \in[n r .(n+1) r]$. o suponhanos anhecirla a solução sobre o intervalo $[(n-1) r, m r]$, a fual denotarenos por $x_{n-1}$. Determinemos a soluçăo $x_{n}$ sobre $[m,(n+1) r]$, como segue:

$$
\left\{\begin{array}{l}
\dot{x}_{n}(t)=f\left(t, x_{n-1}(t-r)\right) \\
x_{n}(n r)=x_{n-1}(n r)
\end{array}\right.
$$

dondesegue,

$$
x_{n}(t)=x_{n-1}(n r)+\int_{n r}^{r} f\left(\tau, x_{n-1}(\tau-r)\right) d \tau,
$$

(com $t \in[n r,(n+1) r]$.

Agora, passaremos à definiçào de equaçōes diferenciais funcionais retardadas.

Consideremos um real nào negativo $r$ e $C=C\left([-r, 0], \mathbb{R}^{n}\right)$ o espaço das funções coutínuas de $[-r, 0]$ sobre $\mathbb{R}^{n}$. O espaço $C$ é um espaço de Banach com a norma:

$$
|\phi|=\sup _{-r \leq \theta \leq 0}|\phi(\theta)| \text {, }
$$

onde no sogundo nembro, $|\cdot|$ demotia a norna nsual de $\mathbb{R}^{n}$

Consicleremos um real $A>00 x:\left[t_{0}-r, t_{0}+A\right) \rightarrow \mathbb{R}^{n}$ mma função contínua. Lntáo. para todo $t \in\left[t_{0}, t_{0}+A\right]$, definimos $x_{t} \in C\left([-r, 0], \mathbb{R}^{n}\right)$ por:

$$
\begin{aligned}
& x_{t}:[-r, \theta] \longrightarrow \mathbb{R}^{n}, \\
& r_{t}(\theta)=r(t-\theta) .
\end{aligned}
$$

Definição 1.2.1 $S$ eja $f:[0, \infty) \times C\left([-r, 0], \mathbb{R}^{n}\right) \rightarrow \mathbb{R}^{n}$ é uma funçcio dada. definimos

$$
\dot{x}(t)=f\left(t, x_{t}\right)
$$

como sendo a equação diferencial funcional retardada (EDFR) sobre $[0, \infty)$.

Definiçāo 1.2.2 Uma funcão $x(t)$, contínua em $\left[t_{0}-r, t_{0}+A\right), \operatorname{com} A>0, t_{0} \geq 0$, é uma solução da equaçăo (1.3) se for difcrenciával em $\left[t_{0}, t_{0}+A\right)$ e $\dot{x}(t)=f\left(t, x_{t}\right)$, para $t_{0} \leq t \leq t_{0}+A$.

Definição 1.2.3 Sejam $t_{0} \geq 0, A>0$ e $\psi \in C\left([-r, 0], \mathbb{R}^{n}\right)$. Urua função $x(t)$ contínua em $\left[t_{0}-r, t_{0}+A\right)$, diferenciável em $\left[t_{0}, t_{0}+A\right)$ é uma solução de (1.3) com condição inicial $\psi$ em $t_{0}$ se:

i) $x_{t_{0}}(\theta)=\psi(\theta)$, para $\theta \in[-r, 0]$

ii) $i(t)=f\left(t, x_{t}\right)$, prorat $\in\left[t_{0}, t_{0}+A\right)$.

\section{Observações:}

- Não precisamos exigir que a função $x(t)$, definida sobre $\left[t_{0}-r, t_{0}+A\right)$, seja diferenciável en $t_{0}$, basta que $x(l)$ seja diferenciável à direita desse ponto.

- Quando $r=0$, a equação diferencial funcional retardada se reduz à cquação diferencial ordinária. 
Definição 1.2.4 Seja $\phi \in C\left([-r, 0], \mathbb{R}^{\prime \prime}\right)$. Dizcmos que a funçáo $f(t$, bi) satisfaz a condição de Lipschitz ou é lipschitziana relativamente a $\phi$ em $C\left([-r, 0], \mathbb{R}^{n}\right)$, st rriste unt ronstante $L \geq 0$, tal que':

$$
\left|f\left(t, \phi_{2}\right)-f\left(t, \phi_{1}\right)\right| \leq L\left|\phi_{2}-\phi_{1}\right| .
$$

mmo $</<\infty C \phi_{1}, \phi_{2} \in C\left([-r, 0] \mathbb{R}^{n}\right)$. O numero L chama-se constante de Lipschitz.

Definiçāo 1.2.5 Para $H \in \mathbb{R}$, com $0<H<\infty$, denotamos por $C_{H}$ o conjunto dus funcries rontinuas definidas $\mathrm{cm}[-r, 0]$, com norma menor que $H$. ou seja.

$$
C_{H}=\left\{\phi \in C\left([-r, 0], \mathbb{R}^{n}\right):|\phi|<H\right\} .
$$

Definiçāo 1.2.6 Dizemos que a equação $f\left(t, x_{t}\right)$ é localmente lipschitziana relativamente a $\phi$ em $[0, \infty) \times C\left(\mid-r, \mathbb{R}^{n}\right)$ se, para todo $\tau>0$ e todo real positivo $H$, existe uma constante $L=L(\tau, H) \geq 0$ tal que:

$$
\left|f\left(l, \phi_{2}\right)-f\left(t, \phi_{1}\right)\right| \leq L\left|\phi_{2}-\phi_{1}\right|,
$$

para todo $0 \leq t \leq \tau$ e $\phi_{1}, \phi_{2} \in C_{H}$.

Definição 1.2.7 Consideremos $X$ um espaço métrico e $|\cdot|$ a norma induzida pela métrica. Uma aplicação $T: X \longrightarrow X$ chama-se contração quando existe $\lambda \in \mathbb{R}$, com $0 \leq \lambda<1$. lal que:

$$
|T(u)-T(v)| \leq \lambda|u-v|,
$$

para todo $u, \| \in X$.

Teorema 1.2.1 (Teorema do Ponto Fixo de Banach). Sejam $X$ um espaço métrico rompleto e $T: X \longrightarrow X$ uma contraçào. Então, existe um único $u \in X$ tal que $T(u)=u$.

Demonstração:

$\Lambda$ demonstração pode ser encontrada cm Zeidler [17].

Teorema 1.2.2 (Existência e Unicidade de Soluções). Seja $f(t, \phi)$ contínua e localmente lipschitziana relativamente a $\phi$ em $[0, \infty) \times C\left([-r, 0], \mathbb{R}^{\prime \prime}\right)$, com constante de Lipschitiz L. Então, para qualquer $t_{0} \geq 0$ e $\psi \in C\left([-r, 0], \mathbb{R}^{n}\right)$ fixos; existem $A>0 e$ uma única funçao $x(t)$ definida $\mathrm{cm}\left[t_{0}-r, t_{0}+A\right)$, a qual é solução da equação diferencial funcional (1.3) com condição inicial $\psi$ em $t_{0}$

\section{Demonstração:}

Consideremos o seguinte conjunto:

$$
F=\left\{x \in C\left(\left[t_{0}-r, t_{0}+A\right] ; \mathbb{R}^{n}\right):|x| \leq H, x\left(t_{0}+\theta\right)=\psi(\theta), \theta \in[-r, 0]\right\} .
$$

onde $H>0$ é tal que $|\psi(0)|<H,|\tau|=\left|t_{0}+A\right|<H e A>0$ será fixado posteriomente.

Inicialmente, mostraremos que $F$ é um espaço métrico completo. Para isso, mostraremos que $F$ é fechado, isto é, toda seqüência,

$$
\left(x_{n}\right)_{n \in \mathbb{N}}, x_{n} \in F, n \in \mathbb{N}
$$


tal yule

$$
x_{n} \stackrel{n \rightarrow \infty x}{\longrightarrow} x
$$

'mitan, lemos glue $x \in l$.

('ome)

$$
x=\lim _{n \rightarrow \infty} x_{n}
$$

obtemes da contimuidade da lunçào módulo gue

$$
|x|=\lim _{n \rightarrow \infty}\left|x_{n}\right|
$$

Para cula $n \in \mathbb{N}$, temos que $\left|x_{n}\right| \leq H$, pois, $x_{n} \in F$. Então, segue que $|x| \leq H$.

Também conseguimos $x\left(t_{0}+\theta\right)=\psi(\theta)$, mua vez que, $x_{n}\left(t_{0}+\theta\right)=\psi(\theta)$, para $\theta \in[-r, 0]$ (. todo $n \in \mathbb{N}$.

Portanto, $F$ é um espaço métrico completo.

Consideremos a aplicação $T: F \rightarrow C\left(\left[t_{0}-r, t_{0}+A\right] ; \mathbb{R}^{n}\right)$ definida por:

$$
\begin{array}{ll}
(T x)\left(t_{0}+\theta\right)=\psi(\theta), & \text { para } \theta \in[-r, 0], \\
(T \cdot x)(t)=\psi(0)+\int_{t_{0}}^{t} f\left(s, x_{s}\right) d s, & \text { para } t \in\left[t_{0}, t_{0}+A\right] .
\end{array}
$$

Mostraremos que $T$ é uma aplicação de $F$ em $F$, comsiderando $A$ convenientemente. Para isso, temos que verificar que $|(T x)(t)| \leq I I$, para todo $x \in F$ e $t_{0} \leq t \leq t_{0}+A$.

Usando a definição de T: conseguimos:

$$
|(T x)(t)| \leq|\psi(0)|+\int_{t_{0}}^{t}\left|f\left(s, x_{s}\right)\right| d s \leq|\psi(0)|+\int_{t_{0}}^{t_{0}+A}\left|f\left(s, x_{s}\right)\right| d s .
$$

para $t_{0} \leq t \leq t_{0}+A$

Observemos que, para $t_{0} \leq t \leq t_{0}+A$.

$$
\left|x_{s}\right|=\sup _{-r \leq \theta \leq 0}|x(s+\theta)| \leq|x| \leq H
$$

antão, temos que,

$$
\left|f\left(s, x_{s}\right)\right| \leq\left|f\left(s, x_{s}\right)-f(s, 0)\right|+|f(s, 0)| \leq L\left|x_{s}-0\right|+K \leq L H+K
$$

(onde

$$
K=\sup _{t_{0} \leq s \leq t_{0}+A}|f(s, 0)| e L=L\left(t_{0}+A, H\right)
$$

Daí, obtemos,

$$
|(T x)(l)| \leq|\psi(0)|+[L H+K] \int_{t_{0}}^{t_{0}+A} d s=|\psi(0)|+\left[L H+K^{*}\right] A
$$

vara $t_{0} \leq t \leq t_{0}+A=\tau$.

Por ontro lado, como $|\psi(0)|<H$, tomando $A \leq \frac{I I-|\psi(0)|}{L H+K}$, temos que $|T x| \leq H$.

Concluímos assin, que $T x \in F$, ou seja, $T$ é una aplicação de $F \operatorname{cm} F$. 
Agora. tomando $A$ dado por:

$$
A<\min \left\{\frac{H-|\psi(0)|}{L I I+K} ; \frac{1}{L}\right\}
$$

mestramenos que $T$ é uma contração de $F$ em $F$.

Dinlos $x, y \in F$. 1.emos

$$
\begin{array}{ll}
(T x)\left(t_{0}+\theta\right)=(T y)\left(t_{0}+\theta\right)=\psi(\theta), & \text { para } \theta \in[-r, 0], \\
(T x)(t)=\psi(0)+\int_{t_{0}}^{t} f\left(s, x_{s}\right) d s, & \text { para } \theta \in\left[t_{0}, t_{0}+A\right], \\
(T y)(t)=\psi(0)+\int_{t_{0}}^{t} f\left(s, y_{s}\right) d s, & \text { para } \theta \in\left[t_{0}, t_{0}+A\right],
\end{array}
$$

corre $t_{0} \leq t \leq t_{0}+A$.

Então, temos que $|(T x)(t)-(T y)(t)|=0$, se $t_{0} \cdots r \leq t \leq t_{0}$, c

$$
|(T x)(t)-(T y)(t)| \leq \int_{t_{0}}^{t}\left|f\left(s, x_{s}\right)-f\left(s, y_{s}\right)\right| d s \leq \int_{t_{0}}^{t_{0}+A} L\left|x_{s}-y_{s}\right| d s,
$$

set $t_{0}<t<t_{0}+A$

Observemos (yue:

$$
\left|x_{s}-y_{s}\right|=\operatorname{sip}_{r \leq \theta \leq 0}\left|x_{s}(\theta)-y_{s}(\theta)\right| \leq|x-y|
$$

Assim, conseguimos

$$
|(T x)(t)-(T y)(t)| \leq L|x-y| \int_{t_{0}}^{t_{0}+A} d s=A L|x-y|
$$

Portanto, obtemos que $T$ é uma contração, uma vez que $A L<1$.

Por úllimo, aplicando o Teorema do Ponto Fixo de Banach, temos que existe uma única função $x \in F$ tal que $(T x)(t)=x(t)$, para $\iota_{0}-r \leq t \leq t_{0}+A$, ou seja,

$$
\begin{array}{ll}
(T x)\left(t_{0}+\theta\right)=x\left(t_{0}+\theta\right)=\psi(\theta), & \text { para } \theta \in[-r, 0], \\
(T x)(t)=x(t)=\psi(0)+\int_{t_{0}}^{t} f\left(s, x_{s}\right) d s, & \text { para } t \in\left[t_{0}, t_{0}+A\right] .
\end{array}
$$

Disso, conclúmos que:

$$
\begin{aligned}
x_{t_{0}}(\theta) & =x\left(t_{0}+\theta\right)=\psi(\theta), & & \text { para } \theta \in[-r, 0], \\
\dot{x}(t) & =f\left(t, x_{t}\right), & & \text { para } t \in\left[t_{0}, t_{0}+A\right] .
\end{aligned}
$$

\section{Observação:}

Sujondo que função $f(t, \phi)$ seja apenas contínua, podemos provar a existencia, mas 


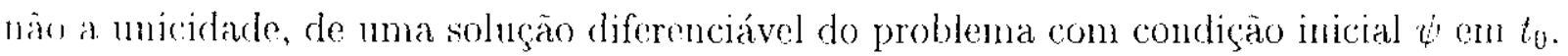
definida sobre $\left[t_{0}-r, t_{0}+A\right)$, conn $A$ suficientemente perfueno. Podenos provar esse caso usando o Terorema do Ponto Fixo de Schander.

\subsection{Semigrupos para Equações Lineares Autonòmas}

Nestia seçàn, faremos uma breve introdução à teoria de semigrupos para equaçōes liferenciais funcionais lineares autônomas. As clemonstraçues dos resultados que enunciaremos nesta seção poderào ser encontradas en Hale \& Luntel [7].

Considerenos a equlaçāo diferencial funcional retardada linear autônoma,

$$
\therefore(t)=L\left(x_{t}\right)
$$

onde $L$ ci una aplicação linear contínua de $C=C\left([-r, 0], \mathbb{R}^{n}\right)$ em $\mathbb{R}^{n}$.

Pelo Teorema de Representação de Riez, podemos reescrever $L$ na forma:

$$
L(\phi)=\int_{-r}^{0}[d \eta(\theta)] \phi(\theta), \phi \in C,
$$

onde $\eta(\theta), \theta \in[-r, 0]$, é una matriz de ordem $n$, cujos elementos são de variaçào limitada, normalizarla a fin de que $\eta$ seja continua à escuerda sobre $(-r, 0)$, ver [11].

Definição 1.3.1 Seja $\mathcal{B}$ um espaço de Banach real. Un semigrupo fortemente continuo de operadores linerares. que demotaremos por $C_{0}$-semigrupo, é uma família a um paranctro,

$$
T^{\prime}(t): \mathcal{B} \longrightarrow \mathcal{B}, t \geq 0
$$

ds: operadores lineares limitados que satisfaz as propriedades:

i) $T(0)=1$;

ii) $T\left(t_{1}+t_{2}\right)=T\left(t_{1}\right) T\left(t_{2}\right), t_{1}, t_{2} \geq 0$;

iii) $\lim _{t \rightarrow 0}\|T(t) \phi-\phi\|=0, \phi \in \mathcal{B}$.

Para todo $C_{0}$-senigrupo $T(t)$, podemos associar mon gerador infinitesimal $A_{0}$, definido per:

$$
\begin{aligned}
& A_{0}: D\left(A_{0}\right) \longrightarrow \mathcal{B}, \\
& A_{0} \phi=\lim _{t \rightarrow 0} \frac{1}{t}[T(t) \phi-\phi], \phi \in D\left(A_{0}\right),
\end{aligned}
$$

mute $D\left(A_{0}\right)$ b o conjunto das funções $\phi$ para as quais o limite acima existe, ou seja,

$$
D\left(A_{0}\right)=\left\{\phi \in \mathcal{B}: \exists \lim _{t \rightarrow 0} \frac{1}{t}[T(t) \phi-\phi]\right\} .
$$

Lema 1.3.1 Se $T(t)$ é um $C_{0}$-semigrupo sobre $\mathcal{B}$, entào temos:

(i) Para toda $\phi \in \mathcal{B}$, a aplicaçăo $t \rightarrow T(t) \phi$ é contínua de $\mathbb{R}^{+}$em $\mathcal{B}$;

(ii) $A_{0}$ é um operador fechado densamente definido; 
(iii) Para toda $\phi \in \mathcal{B}$, a aplicacio $t \rightarrow T(t) \phi$ satisfaz a equacrio diferencial.

$$
\frac{d}{d t} T(t) \phi=\cdots A_{0} T(t) \phi=T(t) A_{0} \phi
$$

() oprudor lincar $L: C \rightarrow \mathbb{R}^{n}$ é linitado, on seja, existe uma constante $K \geq 0$ tal (1116:

$$
|L(\phi)| \leq K|\phi|,
$$

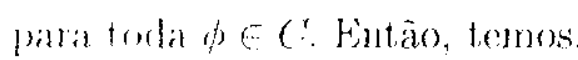

$$
\left|L\left(\phi_{1}\right)-L\left(\phi_{2}\right)\right|=\left|L\left(\phi_{1}-\phi_{2}\right)\right| \leq k^{\prime}\left|\phi_{1}-\phi_{2}\right|,
$$

para torla $\phi_{1}, \phi_{2} \in C$.

Logo, segue que $L$ é Lipschtiziana, o portanto, pelo Teorema 1.2 .2 , para cada $\phi \in C$, exister una única solução $x(., \phi)$ da equação (1.4).

Definiçāo 1.3.2 Considercmos o cspaço de Banach $C=C\left(\{-r, 0], \mathbb{R}^{n}\right)$. Seja $\phi \in C$ c $x(., \phi)$ a única soluçào da equaçào diferencial funcional linear autônoma (1.4), com condicio inicial $\phi$ em $t_{0}=0$, ou seja

$$
\begin{aligned}
& x(t)=L x_{t}, \\
& x_{0}(\theta)=\phi(\theta), \theta \in[-r, \theta],
\end{aligned}
$$

entrio definimos o operador solução $T(t), t \geq 0$, por:

$$
T(t) \phi=x_{t}(., \phi) .
$$

Agora. cmunciaremos alguns resultados para o operador solução.

Lema 1.3.2 O operador soluçäo $T(t), t \geq 0$, defunido pela relaçăo (1.5), é um $C_{0}$-semigrupo, com o grerador infinitesimal dado por:

$$
\left\{\begin{array}{l}
A_{0}: D\left(A_{0}\right) \longrightarrow C, \\
A_{0} \phi=\dot{\phi}=\frac{d \phi}{d \theta},
\end{array}\right.
$$

form

$$
D\left(A_{0}\right)=\left\{\phi \in C: \frac{d \phi}{d \theta} \in C, \dot{\phi}(0)=L(\phi)\right\}
$$

Al'm disso, o operador $T(t)$ é completamente contínuo para $t \geq r$, isto é. o operador $T(t), t>r$, continuo e aplica conjuntos fechados em conjuntos relativamente compactos.

Agora introduriremos a equação adjunta associada à equação diferencial funcional retardada linear autônoma (1.4).

Tomemos $B_{0}$ o espaço das funções $\psi:(-\infty, 0] \rightarrow \mathbb{R}^{n^{*}}$ que são constantes em $(-\infty,-r]$, de variação limitada sobre $[-r, 0]$, contínuas à esquerda sobre $(-r, 0)$, e nulas no zero com a norma $\operatorname{Var}(-r, 0\})$, onde $\mathbb{R}^{n^{*}}$ é o espaço $n$-dimensional dos vetores linhas. 


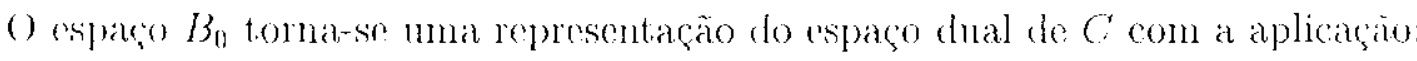

$$
<f, \phi>-\int_{-r}^{\theta} d[\eta(\theta)] \phi(\theta), f \in B_{0}, \phi \in C
$$

Dofinimos a equaça arljunta formal por:

$$
y(s)+\int_{s}^{t} y(\tau) \eta(\tau, s-\tau) d \tau=\text { constante, } s \leq t-r
$$

onde y é uma solução que se anula on $[t, \infty)$, satisfaz a equação adjuntia em $(-\infty, t-r]$ a tal que $y(t+\theta)=\psi(\theta),-r \leq 0 \leq 0$, com $\psi \in B_{0}$.

O) operador adjunto $A^{*}$ do operador $A_{0}$ é definido por: $f \in D\left(A^{*}\right)$ se, e somente se, rxiste $g \in B_{0}$ tal que

$$
<f, A_{0} \phi>=<g, \phi>
$$

para toda $\phi \in D\left(A_{0}\right)$ e no caso, $A^{*} f=g$.

Lema 1.3.3 O operador adjunto $A^{*}: D\left(A^{*}\right) \rightarrow B_{0}$ é dado por:

$$
\begin{aligned}
& D\left(A^{*}\right)=\left\{f \in B_{0}: \frac{d f}{d \theta} \in B_{0}\right\}, \\
& A^{*} f(\theta)=f(0-) \eta(\theta)-\frac{d f(\theta)}{d \theta},-r \leq \theta \leq 0 .
\end{aligned}
$$

Fstudaremos também. o semigrupo associado à equação transposta da equaçäo diforencial funcional retardada linear autônoma (1.4).

Seja $C^{*}=C\left([0, r], \mathbb{R}^{n^{*}}\right)$, e para $s \in[0, \infty)$, tomemos $y^{s}$ um elemento en $C^{*}$ definido por:

$$
y^{s}(\xi)=y(-s+\xi), \xi \in[0, r] .
$$

O sistema transposto da equação (1.4) é definido por:

$$
\begin{aligned}
& \dot{y}(s)=-\int_{-r}^{0} y(s-\theta) d[\eta(\theta)], s \leq 0, \\
& y^{0}(\xi)=\psi(\xi), \xi \in[0, r],
\end{aligned}
$$

$\operatorname{com} \psi \in C^{*}$

Definição 1.3.3 Consideremos o espaço de Banach $C^{*}=C\left([-r, 0], \mathbb{R}^{n^{*}}\right)$. Seja $\psi \in C^{*}$ $r y(., y)$ a úmica solucia da equaço transposta (1.6), com condiçăo inicial $\psi$ em $t_{0}=0$ Definimos o operador transposto $T^{7}(t), t \geq 0$, dado por:

$$
T^{\mathrm{T}}(t) \psi=y_{s}(., \psi)
$$

Lema 1.3.4 O operador solução $T^{T}(s), s \geq 0$, da equação transposta, definido pela relaçâo (1.7) é um $C_{0}$-semigrupo com gerador infinitesimal $A_{0}^{T}$, dado por: 


$$
\begin{gathered}
A_{0}^{T}: D\left(A_{0}^{T}\right) \longrightarrow C^{*}, \\
A_{0}^{T} \psi=-\frac{d y}{d \xi}, \\
I)\left(A_{0}^{T}\right)=\left\{y \in C^{*}: \frac{d \psi^{\prime}}{d \xi} \in C^{*}, \frac{d y^{\prime}}{d \xi}(0)=-\int_{-r}^{0} \psi(-\theta) d[\eta(\theta)]\right\} .
\end{gathered}
$$

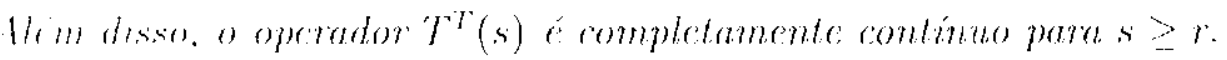

\subsection{Decomposição de $C$}

Nesta seção, vamos decompor o espaço das funçōes contínuas de $[-r, 0]$ sobre $\mathbb{R}^{n}$ em soma direta de dois espaços invariantes pelo gerador infinitesimal $A_{0}$. sendo que un deles ‘ do dimensão finita, e está associado a $1 \mathrm{~m}$ subconjunto finito e não vazio desse gerador infinitesimal. Para isso, precisamos determinar a natureza do espectro do $C_{0}$-semigrupo daclo polo operador solução $T(t)$ e de seu gerador infinitesimal $A_{0}$.

Para introduzirmos o espectro de um operador, precisamos considerar espaços de Bamali complexos. Sejam $\mathcal{B}_{\mathbb{C}}$ o espaço de Banach real complexificado e

$$
B_{\mathbb{C}}: D\left(B_{\mathbb{C}}\right) \subset \mathcal{B}_{\mathbb{C}} \rightarrow \mathcal{B}_{\mathbb{C}}
$$

o operador lincar complexificado. Isso significa que existe um espaço de Banach real $\mathcal{B}$ e inim operator

$$
B: D(B) \subset \mathcal{B} \rightarrow \mathcal{B}
$$

t.al qu"

$$
\mathcal{B}_{\mathfrak{C}}=\mathcal{B}+i \mathcal{B} \text { e } B_{\mathbb{C}}\left(b_{1}+i b_{2}\right)=B\left(b_{1}\right)+i B\left(b_{2}\right)
$$

para $b_{1}, b_{2} \in D(B)$. Em $\mathcal{B}_{\mathbb{C}}$, podemos definir o complexo conjugado:

$$
\overline{b_{1}+i b_{2}}=b_{1}-i b_{2}
$$

Sempre que não houver problemas, escreveremos $\mathcal{B}$ para $\mathcal{B}_{\mathbb{C}}$ e $B$ para $B_{\mathbb{C}}$.

Definição 1.4.1 O conjunto resolvente $\rho(B)$ do operador $B$ é o conjunto de valores $\lambda$ no plano complexo para os anais o operador $(\lambda I-B)$ é inversinel, onde o inverso é limitado com domínio denso em $\mathcal{B}$. Esse inverso será denotado por:

$$
R(\lambda, B)=(\lambda I-B)^{-1}, \lambda \in \rho(B),
$$

c chamado de resolvente de $B$

Definição 1.4.2 $O$ complementar do conjunto resolvente $\rho(B)$ do operador $B$ no plano complexificado é chamado de espectro de $B$ e denotamos por $\sigma(B)$.

$O$ espectro de um operador pode se constituir de três tipos difcrentes de pontos:

- o espectro residual $\sigma_{R}(B)$, o qual é formado pelos pontos do plano complexo $\lambda$, para os quais $R(\lambda, B)$ existe, mas o domínio $D(R(\lambda, B))$ não é denso em $\mathcal{B}$.

- ospectro contínuo $\sigma_{C}(B)$, o qual é formado pelos pontos do plano complexo $\lambda$, 


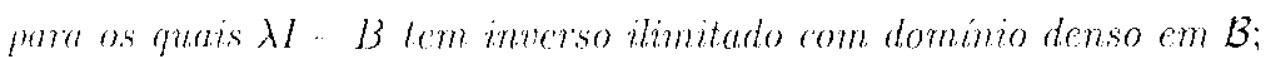

- o espctro pontual $\sigma_{p}(B)$, o qual é formado polos pontos do plano complero $\lambda$. para os quais $\lambda I-B$ nio tem inverso.

Definição 1.4.3 I'ark $\lambda \in \sigma_{p}(B)$, o autoespaço generalizado de $\lambda$, demoludo por $\mathcal{A}_{,}(B)$, i o menor subespreso de $\mathcal{B}$ que rontim os clementos de

$$
\bigcup_{k=1}^{\infty} \operatorname{ser}\left((\lambda I \cdots B)^{k}\right)
$$

A dimensào do $\mathcal{M}_{\lambda}(B)$ é chamada de multiplicidade algébrica de $\lambda$ e o menor inteiro positivo $\mathrm{k}$ tal que

$$
\operatorname{Ker}\left((\lambda I-B)^{k}\right)=\operatorname{Ker}\left((\lambda I-B)^{k+1}\right)
$$

Chomado do ascendente de $\lambda$

Os pontos do espectro pontual de $B$ com autoespaço de dimensäo finita säo chamados autovalores do tipo finito ou autovalores normais.

Lema 1.4.1 Se $A_{0}$ r o operador definado pelo Lema 1.3.2, cntào o cepertro de $A_{0}$ é iqual ai espectro pontual de $A_{0}$, ou seja,

$$
\sigma\left(A_{0}\right)=\sigma_{p}\left(A_{0}\right)
$$

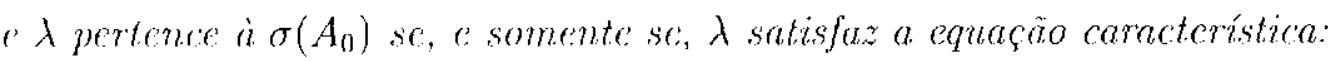

$$
\operatorname{det}(\Delta(\lambda))=0, \Delta(\lambda)=\lambda I-\int_{-r}^{0} e^{\lambda \theta} d[\eta(\theta)] .
$$

Alcim disso, para $\lambda \in \sigma\left(A_{0}\right)$, o autoespaço generalizado $\mathcal{M}_{\lambda}\left(A_{0}\right)$ é de dimensià finita. riste wm inteiro $k$ tal que

$$
\mathcal{M}_{\lambda}\left(A_{0}\right)=\operatorname{Ker}\left(\left(\lambda I-A_{0}\right)^{k}\right),
$$

Pemos a decomposiçáa em soma direta

$$
C=\operatorname{Ker}\left(\left(\lambda I-A_{0}\right)^{k}\right) \oplus \operatorname{Im}\left(\left(\lambda I-A_{0}\right)^{k}\right) .
$$

\section{Observação:}

Desse lema, temos que $\lambda \in \sigma\left(A_{0}\right)$ implica que $\mathcal{M}_{\lambda}\left(A_{0}\right)$ é de dimensão finita e $\mathcal{M}_{\lambda}\left(A_{0}\right)=$ $\operatorname{Lier}\left(\left(\lambda I-A_{0}\right)^{k}\right)$ para algum inteiro $k$. Como $A_{0}$ comuta com $\lambda I-A_{0}$, entäo, o subespaço $\mathcal{M}_{\lambda}\left(A_{0}\right)$ é invariante por $A_{1}$.

Seja $d$ a dimensão de $\mathcal{M}_{\lambda}\left(A_{0}\right)$ e $\Phi_{\lambda}=\left\{\phi_{1}^{\lambda}, \ldots, \phi_{d}^{\lambda}\right\}$ uma base para $\mathcal{M}_{\lambda}\left(A_{0}\right)$.

Como $A_{0} \mathcal{M}_{\lambda}\left(A_{0}\right) \subseteq \mathcal{M}_{\lambda}\left(A_{0}\right)$, temos,

$$
\left\{\begin{aligned}
A_{0} \phi_{1}^{\lambda} & =b_{11} \phi_{1}^{\lambda}+\cdots+b_{1 d} \phi_{d}^{\lambda} \\
A_{0} \phi_{2}^{\lambda} & =b_{21} \phi_{1}^{\lambda}+\cdots+b_{2 d} \phi_{d l}^{\lambda} \\
& \vdots \\
A_{0} \phi_{d}^{\lambda} & =b_{d 1} \phi_{1}^{\lambda}+\cdots+b_{d, d} \phi_{d}^{\lambda} .
\end{aligned}\right.
$$


I.1)

$$
\left[A_{0} \phi_{1}^{\lambda}, \ldots . A_{0} \phi_{d d}^{\lambda}\right]=\left[\phi_{1}^{\lambda}, \ldots, \phi_{d}^{\lambda}\right] \cdot\left[\begin{array}{cccc}
b_{11} & b_{21} & \cdots & b_{d 1} \\
b_{12} & b_{22} & \cdots & b_{d 2} \\
\vdots & \vdots & \ddots & \vdots \\
b_{1 d} & b_{2 d} & \cdots & b_{t d}
\end{array}\right] .
$$

Assim. obtemos clo fato de $A_{0} \mathcal{M}_{\lambda}\left(A_{0}\right) \subseteq \mathcal{M}_{\lambda}\left(A_{0}\right)$, que existe uma matriz $B_{\lambda}$ de ordem "1 tal 'plul:

$$
A_{0} \Phi_{\lambda}=\Phi_{\lambda} B_{\lambda} .
$$

Lema 1.4.2 O vinico autovalor da matriz $B_{\lambda} \dot{e} \lambda$.

Da definição do operador $A_{0}$ no Lema 1.3 .2 , e da relação

$$
A_{\theta} \Phi_{\lambda}=\Phi_{\lambda} B_{\lambda}
$$

temos que

$$
\Phi_{\lambda}(\theta)=\Phi_{\lambda}(0) e^{B_{\lambda} \theta}, \theta \in[--r, 0] .
$$

Do Iscma 1.3.1, item (iii), obtemos

$$
T(t) \Phi_{\lambda}=\Phi_{\lambda} e^{B_{\lambda} t}, t \geq 0
$$

"porlanto, conseguiunes:

$$
T(t) \Phi_{\lambda}(\theta)=\Phi_{\lambda}(0) e^{B_{\lambda}(\theta+t)}, \theta \in[-r, 0], t \geq 0
$$

Essa relação nos permite definir o operador $T(t)$ sobre o autoespaço generalizado $\mathcal{M}_{\lambda}\left(A_{0}\right)$ para todos os valores de $t \in(-\infty, \infty)$, e portanto, sobre o autosspaço generalizado de um autovalor, a equação (1.4) tem a mesma estrutura de una equação diferencial ordinária.

Sendo assim, temos do Lema 1.3.1, $T(t) A_{0} \phi=A_{0} T(t) \phi$, para toda $\phi \in C$, entào $R\left(\left(\lambda l-A_{0}\right)^{k}\right)$ é invariante por $T(t)$.

Repetindo o processo acima, obtemos o seguinte tcorema.

Teorema 1.4.1 Suponhamos $\Lambda$ um conjunto finito e não vazio $\left\{\lambda_{1}, \ldots, \lambda_{p}\right\}$ de autovalores da. equação (1.4) e sejam $\Phi_{\Lambda}=\left\{\Phi_{\lambda_{1}}, \ldots, \Phi_{\lambda_{p}}\right\}, B_{\Lambda}=\operatorname{diag}\left(B_{\lambda_{1}}, \ldots, B_{\lambda_{p}}\right)$, onde $\Phi_{\Lambda_{j}}$ é uma base do autoespaço generalizado de $\lambda_{j}$ e $B_{\lambda_{j}}$ é a matriz definida por $A_{0} \Phi_{\lambda_{j}}=\Phi_{\lambda_{j}} B_{\lambda_{j}}$. $j=1,2, \ldots, p$. Entào, o único autovalor de $B_{\lambda_{j}}$ ć $\lambda_{j}$ e, para todo vetor u de mesma dimensaio que $\Phi_{\Lambda}$, a soluçào $T(t) \Phi_{\Lambda} u$ com condição inicial $\Phi_{\Lambda}$ u em $t_{0}=0$ pode ser defirita em $(-\infty, \infty)$ pela relaçào:

$$
\left\{\begin{array}{l}
T(t) \Phi_{\Lambda} u=\Phi_{\Lambda} e^{B_{\lambda} t} u \\
\Phi_{\Lambda}(\theta)=\Phi_{\Lambda}(0) e^{B_{\Lambda} \theta}, \text { para } \theta \in[-r, 0] .
\end{array}\right.
$$

Alćm disso, criste um subespaço $Q_{\mathrm{A}}$ de $C$ lal que $T(t) Q_{\mathrm{A}} \subseteq Q_{\mathrm{A}}$ para todo $t \geq 0 \mathrm{e}$

$$
C=I_{\Lambda} \oplus Q_{\Lambda}
$$

onde $P=\left\{\phi \in C: \phi=\Phi_{\Lambda}\right.$ u para algum $\left.u\right\}$. 
() Trorema 1.4.1 nos forncce uma análise das soluçues da equação (1.4). Solro os antorspaços generalizados, a equaçăo (1.4) comportia-se essencialmente como muna equação diforencial ordinária e a decomposição de $C$ en dois subespaços invariantes por $A_{0}$ e $T(t)$ nos diz fue podemos separar o comportanento sobre os autoespaços dos outros tipos de (") mpertanuentos.

\subsection{Decomposição de $C$ com a Equação Adjunta}

Nesta seção, introduziremos una forma bilinear que nos permitirá definir explicitamente o espaço $Q$ e detcrminaremos uma projeção de $C$ sobre os autoespaços generalizados. Os resultados que enunciaremos poderão ser encontrados en Hale [6].

Definição 1.5.1 Sejam $\phi \in C$ e $\psi \in C^{*}$, definimos a forma bilinent (., s) sobre $C^{*} \times C$ por:

$$
\begin{aligned}
& (,,): C^{*} \times C \longrightarrow \mathbb{R}^{n}, \\
& (\psi, \phi)=\psi(0) \phi(0)-\int_{-r}^{0} \int_{0}^{\theta} \psi(\xi-\theta)[d \eta(\theta)] \phi(\xi) d \xi .
\end{aligned}
$$

() gerador infinitesimal $A_{0}^{T}$ do operador solução da equação transposta definido no Lema 1.3.4 satisfaz:

$$
\left(\psi, A_{0} \phi\right)=\left(A_{0}^{T} \psi, \phi\right), \psi \in D\left(A_{0}^{T}\right) \subset C^{*}
$$

para toda $\phi \in D\left(A_{0}\right)$.

Outra propriedade interessante ó a seguinte: suponhanos $y(., \psi)$ uma solução da rquação transposta (1.6) sobre $(-\infty, r)$ com condição inicial $\psi$ no zero, ou seja,

$$
T^{T}(t) \psi=y^{t}(., \phi), t \in(-\infty, 0]
$$

$\operatorname{com} \psi \in D\left(A_{0}^{T}\right)$.

Então, $T^{T}(t)$ tem as mesmas propriedades do operador solução $T(t)$ associado à "ruaçĩo $(1.4) \mathrm{O}$

$$
\frac{d T^{T}(\tau) \psi}{d \tau}=-A_{0}^{T} T^{T} \psi=-T^{T} A_{0}^{T} \psi
$$

para toda $\psi \in D\left(A_{0}^{T}\right)$.

Lema 1.5.1 O espectro do operador $A_{0}$ coincide com o espectro do operador $A_{0}^{T}$. O operador $A_{0}^{T}$ tem somente o espectro pontual e para todo $\lambda \in \sigma\left(A_{0}^{T}\right)$, o autocspaço generalizado de $\lambda$ é de dimensão finita.

Lema 1.5.2 Um condiçâ necessária e suficiente para que a equaçăo

$$
\left(A_{0}-\lambda I\right)^{k} \phi=\psi, \psi \in C,
$$

tenha soluçùo $\phi \in C$, ou equivalentemente, que $\psi \in \operatorname{Im}\left(A_{0}-\lambda I\right)^{k}$. é que $(\alpha, \psi)=0$ para todo $x \in \operatorname{Ker}\left(A_{0}^{T}-\lambda I\right)^{k}$.

O próximo lema caracteriza os espaços $\operatorname{Ker}\left(A_{0}-\lambda I\right)^{k} \operatorname{e} \operatorname{Ker}\left(A_{0}^{T}-\lambda I\right)^{k}$. 
Lema 1.5.3 O c.ppaço $\operatorname{Ker}\left(A_{0}-\lambda I\right)^{k}$ é formado pelas funções $\phi \in C$ da forma:

$$
\phi(\theta)=\sum_{j=0}^{k-1} \gamma_{j+1} \frac{\theta^{j} e^{\lambda \theta}}{j !}, \theta \in[-r, 0],
$$

ande $\gamma=\operatorname{col}\left(\gamma_{1}, \ldots, \gamma_{k}\right)$ satisfaz $A_{k} \gamma=0$, e trmbém, o espaco $\operatorname{Ker}\left(A_{0}^{T} \cdots \lambda\right)^{k}$ é formado phlas funcios $\psi^{\prime} \in C^{*}$ da forma:

$$
\psi(s)=\sum_{j=0}^{k-1} \beta_{j+1} \frac{(-s)^{k-j} e^{-\lambda s}}{j !}, s \in[0, r],
$$

onde $\beta=$ limh $a\left(\beta_{1}, \ldots, \beta_{k}\right)$ satisfaz $\beta A_{k}=0$, com

$$
\begin{aligned}
& A_{k}=\left[\begin{array}{cccc}
\Delta(\lambda) & \frac{d \Delta(\lambda)}{d \lambda} & \cdots & \frac{d^{k-1} \Delta(\lambda)}{d \lambda^{k-1}} \\
0 & \Delta(\lambda) & \cdots & \frac{d^{k-2} \Delta(\lambda)}{d \lambda^{k-2}} \\
\vdots & \vdots & \ddots & \vdots \\
0 & 0 & \cdots & \Delta(\lambda)
\end{array}\right] \\
& \Delta(\lambda)=\lambda I-\int_{-r}^{0} e^{\lambda \theta} d[\eta(\theta)] .
\end{aligned}
$$

Teorema 1.5.1 $\operatorname{Sej} a \lambda \in \sigma\left(A_{0}\right)$. Consideremos $\Psi_{\lambda}=\operatorname{col}\left(\psi_{1}, \ldots, \psi_{p}\right)$ e $\Phi_{\lambda}=\left(\phi_{1}, \ldots, \phi_{p}\right)$ bases de $\mathcal{M}_{\lambda}\left(A_{0}^{T}\right)$ e $\mathcal{M}_{\lambda}\left(A_{0}\right)$, respectivamente, e seja $\left(\Psi_{\lambda}, \Phi_{\lambda}\right)=\left[\left(\psi_{i}, \phi_{j}\right)\right], i, j=1,2, \ldots, p$. Entroo, $\left(\Psi_{\lambda}, \Phi_{\lambda}\right)$ é não singular, e desse modo, podemos tomar como sendo a identidade, $e$ mais. a decomposiçáo de $C$ por $\Lambda$, dada no Teorema 1.4 .1 , pode ser escrita explicitamente (omo.

$$
\begin{gathered}
\phi=\phi^{P_{\lambda}}+\phi^{Q_{\lambda}}, \phi^{P_{\lambda}} \in P_{\lambda}, \quad \phi^{Q_{\lambda}} \in Q_{\lambda}, \\
P_{\lambda}:=M_{\lambda}\left(A_{0}\right)=\left\{\phi \in C: \phi=\Phi_{\lambda} b, b \text { é um p-vetor }\right\}, \\
Q_{\lambda}=\left\{\phi \in C:\left(\Psi_{\lambda}, \phi\right)=0\right\}, \\
\phi^{P_{\lambda}}=\Phi_{\lambda} b, \text { onde } b=\left(\Psi_{\lambda}, \phi\right), \\
\phi^{Q_{\lambda}}=\phi-\phi^{P_{\lambda}} .
\end{gathered}
$$

Suponlames que $\left(\Psi_{\lambda}, \Phi_{\lambda}\right)=I$ e que $B_{\lambda}, B_{\lambda}^{*}$ sio as matrizes constantes tais que:

$$
A_{0} \Phi_{\lambda}=\Phi_{\lambda} B_{\lambda} \quad \text { e } A_{0}^{T^{\prime}} \Psi_{\lambda}=B_{\lambda}^{*} \Psi_{\lambda}
$$

ent.ino. temos que $B=B^{*}$ 
Lema 1.5.4 A dimensào do antocspaço associado à $\lambda$ é igual a multiplicidade de $\lambda$ como aron da equaçar caracteristica dada pelo Lema 3.3, on seja,

$$
\operatorname{dim} \mathcal{M}_{\lambda}=\text { multiplicidede de } \lambda \text { como zcro de } \Delta(\lambda) \text {. }
$$

Lema 1.5.5 Sejam $\lambda, \mu \in \sigma\left(A_{0}\right)=\sigma\left(A_{0}^{T}\right), \operatorname{com} \lambda \neq \mu, e \phi \in \mathcal{M}_{\lambda}\left(A_{0}\right), \phi \in \mathcal{M}_{i 1}\left(A_{0}^{T}\right)$, intios. temos gue $(\psi, \phi)=0$.

'Tomemos $\Lambda=\left\{\lambda_{1}, \ldots, \lambda_{p}\right\}$ um conjunto finito de elementos de $\sigma\left(A_{0}\right)$ e seja

$$
P=P_{\Lambda}=\operatorname{ger}\left\{M_{\lambda_{j}}: \lambda_{j} \in \Lambda\right\},
$$

de mancira similar ao feito auteriormente, podemos definir

$$
P^{T}=I_{\Lambda}^{T}=\operatorname{ger}\left\{M_{\lambda_{j}}\left(A_{0}^{T}\right): \lambda_{j} \in \Lambda\right\}
$$

como sendo a autoespaço generalizado da equação trausposta associado a $\Lambda$. Se $\Phi, \Psi$ são hase's para $P . P^{T}$, respectivamente, $e(\Psi, \Phi)=I$, então:

$$
\begin{gathered}
C=P_{\Lambda} \oplus Q_{\Lambda}, \\
P_{\Lambda}:=M_{\Lambda}\left(A_{0}\right)=\left\{\phi \in C: \phi=\Phi_{\Lambda} b, b \text { é ump-vetor }\right\}, \\
Q_{\Lambda}=\left\{\phi \in C:\left(\Psi_{\Lambda}, \phi\right)=0\right\},
\end{gathered}
$$

eportanto, para toda $\phi \in C$,

$$
\begin{aligned}
& \phi=\phi^{P_{\Lambda}}+\phi^{Q_{\Lambda}}, \\
& \phi^{P_{\Lambda}}=\Phi(\Psi, \phi) .
\end{aligned}
$$

\subsection{Teorema das Variedades Invariantes}

Nesta seção, enunciaremos o Teorema das Variedades Invariantes, o qual analisa a existência do tais variedades para equações diferenciais funcionais. Esse teorema analisa o comportamento de soluçöes próximas de um ponto de equilíbrio da equação diferencial funcional autônona e estuda localmente a existência de variedades estáveis, instáveis, centrais,...

Estamos particularmente interessados na existência da variedade central, a qual é apresentada nesse teorema.

Podemos encontrar a demonstração do Teorema das Variedades Invariantes em Hale it Lunel [7].

Seja $\Omega$ uma vizinhança do zero em $C$ e para $\phi \in C$, tomemos a norma:

$$
|\phi|=\sup _{-r \leq \theta \leq 01}|\phi(\theta)| .
$$

Consideremos $C_{b}^{p}\left(\Omega, \mathbb{R}^{n}\right) \subset C^{p}\left(\Omega, \mathbb{R}^{n}\right)$ o conjunto das funçòs de $\Omega$ em $\mathbb{R}^{n}$ que tên derivadas contínuas limitadas até ordem $p$ com respeito a $\phi \in \Omega$. O espaço $C_{b}^{p}\left(\Omega, \mathbb{R}^{n}\right)$ torna-se un espaço de Banach se escolhermos a norma do supremo para todas as derivadas até a ordem $p$. 
Assumiremos que $F \in C_{b}^{1}\left(\Omega, \mathbb{R}^{n}\right)$, e consideremos a equaça diferencial funcional retaritada antonoma:

$$
\dot{r}(t)=F\left(x_{t}\right),
$$

(onn $F(0)=0$, ou seja, 0 ó um ponto de equilíbrio e lincarizando a equação (1.8) em torno (do zeros. tomos:

$$
x(t)=L\left(x_{t}\right)
$$

mule $L \subset \mathcal{L}\left(C, \mathbb{R}^{n}\right) \circ L(\psi)=D_{\phi} F(0) \psi$

Sinpombanos que o é nun ponto de equilibrio nào-hiperbólico da equaçào (1.8), ou seja. ('xiste'm raízes da equaçào característica:

$$
\operatorname{det} \Delta(\lambda)=0, \Delta(\lambda)=\lambda I-L\left(e^{\lambda \cdot I)},\right.
$$

com partes reais nulas.

Podemos decompor o espaço $C$ como segue:

$$
C=U \oplus N \oplus S
$$

onde $U$ ć de dimensão finita c corresponde ao subespaço gerado pelos autoespaços generalizados associados às raízes de (1.10) com partes reais positivas, $N$ também é de dimensão linita e corresponde ao subespaço gerado pelos autoespaços generalizados associados às raízes do (1.10) com partes reais iguais a zero e $S$ corresponde ao subespaço gerado pelos autoc'spaços generalizados associados às raízes de (1.10) com partes reais negativas.

Esssa decomposição de $C$ define três projeções:

$$
\begin{array}{lll}
\pi_{U}: C \longrightarrow U & \pi_{N}: C \longrightarrow N & \pi_{S}: C \longrightarrow S \\
\pi_{U}(U)=U, & \pi_{N}(N)=N, & \pi_{S}(S)=S
\end{array}
$$

$\therefore \pi_{1}+\pi_{N}+\pi_{S}=I$

Sejam $\Phi$ una base de $U$ e $\Psi$ una base de $U^{T}, \operatorname{com}(\Psi, \Phi)=I$, então a projeção $\pi_{U}$ é dada por $\pi_{U}(\phi)=\Phi(\Psi, \phi)$. Similarmente, se $\Phi_{0}$ ć uma base de $N$ e $\Psi_{0}$ é uma base de $N^{T}, \operatorname{com}\left(\Psi_{0}, \Phi_{0}\right)=I$, então a projeção $\pi_{N}$ pode ser dada por $\pi_{N}(\phi)=\Phi_{0}\left(\Psi_{0}, \phi\right)$.

Se $T(t), t \geq 0$, é o $C_{0}$-semigrupo associado à equação linear (1.9), então os espaços $U, N$ e $S$ são invariantes por sob $T(t)$.

Das Seções 7.9 e 9.5 de [7], existem constantes positivas $M, \alpha$, e para todo $\epsilon>0$, existe uma constante positiva $M_{\epsilon}$, tal que para $\phi \in C$, temos:

$$
\begin{aligned}
& \left|T(t) \phi^{U}\right| \leq M e^{\alpha t}\left|\phi^{U}\right|, \operatorname{Var}_{(t, 0]} T(t) X_{0}^{U} \leq M e^{\alpha t}, \quad t \leq 0, \\
& \left|T(t) \phi^{N}\right| \leq M e^{\epsilon|t|}, \operatorname{Var}_{(-t, t)} T(t) X_{0}^{N} \leq M e^{\epsilon|t|}, \quad t \in \mathbb{R}, \\
& \left|T(t) \phi^{S}\right| \leq M e^{-\alpha t}\left|\phi^{S}\right|, \operatorname{Var}_{[0, t)} T(t) X_{0}^{S} \leq M e^{-\alpha t}, \quad t \geq 0 .
\end{aligned}
$$

O conjunto $U$ é caracterizado como o conjunto dos valores iniciais daquelas soluçōes de (1.9) que existem e permanecem limitadas para $t \leq 0$, e a relação (1.11) implica que essas soluçōes aproximanı-se de zero expononcialmente quando $t \rightarrow-\infty$. O conjunto $S$ s caracterizado como o conjunto dos valores iniciais daquelas soluções da equação (1.9) que existem e permanecem limitadas para $t \geq 0$, e essas soluções aproximam-se do zero exponmenalmente quanto $t \rightarrow \infty$. O conjunto $N$ é caracterizado como o conjunto dos 
vatores iniciais daquelas soluçoes da equação (1.9) que existem para $t \in \mathbb{R}$ e croscem nurnos raj)idamente do que qualquer funçào exponenciabmente fixada.

Definição 1.6.1 Dada uma vizinhança $V$ do zero em. $C$, a variedade fortemente estável local $W_{\text {loc }}^{\text {ss }}(0) \stackrel{\text { def. }}{=} W^{\text {sss }}(0, V)$ do ponto de equilibrio 0 da cquaçio $(1.8)$ r a coleça dis: funcrics $\phi \in C$ com a proprictade que a soluça $x_{t}(., \phi) \in C$ parn $t \geq 0$ aproxima-sC du solucrios nulu exponencialmente quando $t \rightarrow \infty$, on seja.

$$
W_{l, c}^{s, s}(0)=\left\{\phi \in C^{\prime}: a_{t}(., \phi) \in V \text { para } t \geq 0 \text { e } x_{t}(., \phi) \rightarrow 0 \text { quanclo } l \rightarrow \infty\right\} .
$$

De modo análogo, definimos a variedade fortemente instável local . que denotamos por $W_{l o c}^{s u}(0) \stackrel{\text { dr.f. }}{=} W^{s u}(0, V)$, como:

$$
W_{l o c}^{s u}(0)=\left\{\phi \in C: x_{i}(., \phi) \in V \text { para } t \leq 0 \text { e } x_{t}(., \phi) \rightarrow 0 \text { quando } t \rightarrow-\infty\right\} .
$$

Definição 1.6.2 Seja $V$ una vizinhança do zero em $C$ : uma variedade central local $W_{\text {loc }}(0) \stackrel{\text { def. }}{=} W^{c}(0, V)$ do ponto de equilíbrio 0 da equaçào (1.8) ć uma $C^{1}$-subvariedade que $\therefore$ um gráfico sobre $V \cap N$ em $C$, tangente à $N$ na origern. é localmente invariante sob "flumo definido pela equaço (1.8), ou seja,

$$
W_{l o c}^{c}(0) \cap V=\{\psi \in C: \psi=\phi+h(\phi), \phi \in N \cap V\},
$$

onde $h: N \rightarrow U \oplus S$ é uma funçào de classe $C^{1}$ com $h(0)=0, D_{\phi}(0)=0$. Portanto, toda ótbita que começa em $W_{\text {loc }}(0)$, continua nesse conjunto enquanto permancce em $V$.

Definição 1.6.3 Dada uma vizinhança $V$ do zero em $C$, uma variedade central estável local $W_{\text {loc }}^{\text {ss }}(0) \stackrel{\text { def. }}{=} W^{\text {css }}(., V)$ do ponto de equilibrio 0 da equaçâo (1.8) a um conjunto em $C$ tal que $W_{l o c}^{c s}(0) \cap V$ é uma subvariedade de classe $C^{1}$ que é um gráfico sobre $V \cap(N \oplus S)$, tangente a $(N \oplus S)$ na origem é é localmente invariante. En outras malawras,

$$
W_{l o c}(0) \cap V=\{\psi \in C: \psi=\phi+h(\phi), \phi \in(N \oplus S) \cap V\},
$$

onde $h: N \oplus S \rightarrow U$ é uma função de classe $C^{l} \operatorname{com} h(0)=0, D_{\phi} h(0)=0$. Portanto, toda órbita que começa em $W_{\text {loc }}^{\text {cs }}(0)$, continua nesse conjunto enquanto permanece em $V$ e além disso, toda órbita que permanece em $V$ para todo $t \geq 0$ deve pertencer à $W_{l o s}^{c s}(0)$.

Do mesmo modo, definimos a variedade central instável local, que denotamos por $W_{l o c}^{r u}(0) \stackrel{\text { def. }}{=} W^{\text {cu }}(0, V)$, do ponto de equilibrio 0 da equaçào $(1.8)$ colocando $t \leq 0 \mathrm{~cm}$ wex de $t \geq 0,(N \oplus U)$ no lugar de $(N \oplus S)$ e $S$ por $N$.

O seguinte teorema diz respeito a existencia de variedades invariantes.

Teorema 1.6.1 Se a função $F$ da equação (1.8) é de classe $C^{k}$, então existe uma vizimhança $V$ do zero en $C$ tal que cada um dos conjuntos $W_{\text {loc }}^{\text {s.s }}(0), W_{\text {loc }}^{\text {sut }}(0), W_{\text {loc }}^{c}(0), W_{\text {loc }}^{c u}(0)$ c. $W_{\text {loc }}(0)$ existem e sio subvariedades de classe $C^{k}$ de $C$. As variedades $W_{\text {loc }}^{\text {ss }}(0)$ e $W_{\text {loc }}^{\text {sin }}(0)$ sio unicamente definidas, cnquanto as variedades $W_{\text {loc }}^{c}(0) W_{\text {loc }}^{c u}(0)$ e $W_{l o c}^{c s}(0)$ nào são. Portanto, todo conjunto invariante da equação (1.8) que permanece em $V$ deve pertencer a. $W_{\text {loc }}^{\text {c }}(0)$.

A partir de agora, usarenos a notação $W^{c}(0)$ para $W_{\text {loc }}^{c}(0)$. 


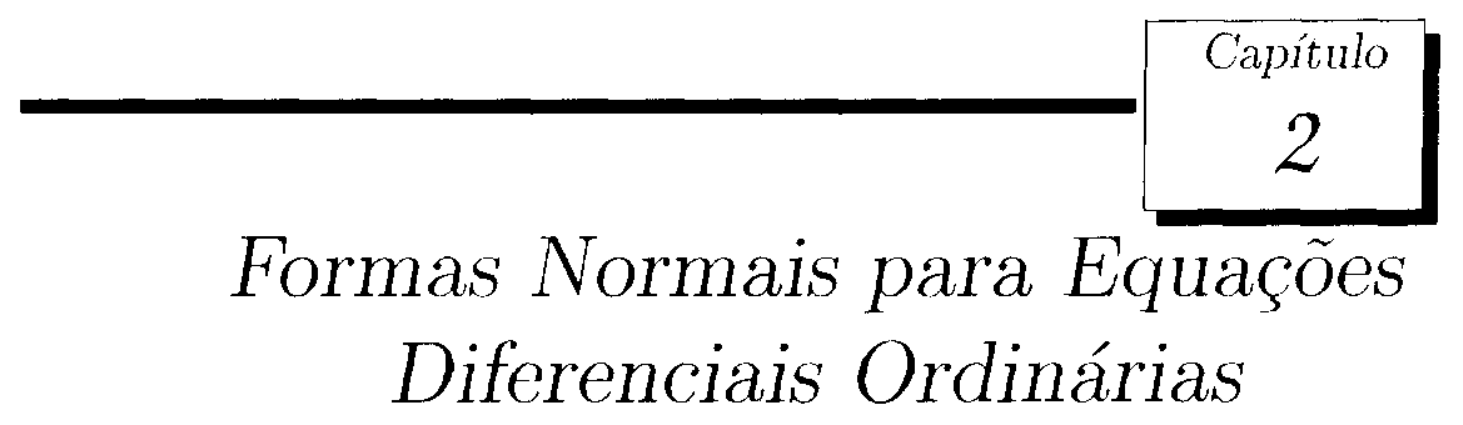

\subsection{Introdução}

() método da forma nomual consiste em encontrar $1 \mathrm{~m}$ sistema de coordenadas, no cual uma equação diferencial ordinária tenha uma forma mais simples. Ao desenvolvernos esse mótoklo. três características devem ser observadas:

$1^{\circ} \mathrm{O}$ método é local no sentido de que as transformações de coordenadas sào consideradas om uma vizinhança de uma solução conhecida; essa solução é, no caso, um ponto fixo.

$2^{\circ}$ Em geral, as transformações de coordenadas são funçōes não lineares, e essas transformaçôes são obtidas através da resolução de uma sequência de problemas lineares.

$3^{\circ}$ A estrutura da forma normal ó determinada pela natureza da 'parte linear' da ratuagão.

\subsection{Formas Normais para Equações Diferenciais Ordinárias}

Consideremos a equação diferencial ordinária:

$$
\dot{w}=G(w) \quad, \quad w \in \mathbb{R}^{n},
$$

onde $G$ é de classe $C^{r}, r \geq 4$. Suponhamos que (2.1) tenha um ponto fixo $w=w_{0}^{0}$

Façamos uma translaçáo do ponto fixo para a origem,

$$
v=w-w_{0}, \quad v \in \mathbb{R}^{\prime \prime},
$$

assim, obtenos:

$$
\dot{v}=G\left(v+w_{0}\right) \equiv I I(v) .
$$

Agora, separemos a parte lincar da parte não linear da seguinte forma:

$$
\dot{v}=D H(0) v+\bar{H}(v),
$$

onde $H(v)=H(v)-D H(0) v$ e $\ddot{H}(v)=O\left(|v|^{2}\right)$.

Seja $T$ a matriz que 'transforma' a matriz $D H(0)$ na forma canônica de Jordan real. Então, fazendo:

$$
v=T x, \quad x \in \mathbb{R}^{n},
$$


oliterimes:

$$
\dot{x}=J x+F(x)
$$

(mule. $\left.I-T^{-1} D\right) /(0) T: F(x)=T^{1} / I(T x)$.

Até o momonto. simplificamos a parte linear da equação diferencial ordinária. Agora. simplificaremos a parle năo linear $F(x)$.

Se tomamos a expansão de Taylor para $F(x)$, temos que:

$$
x=J x+\frac{1}{2} F_{2}(x)+\frac{1}{3 !} F_{3}(x)+\cdots+\frac{1}{(r-1) !} H_{r-1}(x)+O\left(|x|^{r}\right)
$$

onde o trermo $F_{i}(x)$ é formarlo pelos termos de ordem $i$ na expansão de Taylor para $F(x)$.

Considerando a mudança de coordenadas:

$$
r=y+\frac{1}{2} h_{2}(y)
$$

com $h_{2}(y)$ uma função de ordem dois em $y ; h_{2}(y) \in \mathbb{R}^{n}$; obtemos:

$$
\dot{x}=\dot{y}+\frac{1}{2} D h_{2}(y) \dot{y}
$$

r.substit.nindo em (2.4) t.enos que::

$$
\begin{aligned}
y+\frac{1}{2} D h_{2}(y) \dot{y}= & J\left(y+\frac{1}{2} h_{2}(y)\right)+\frac{1}{2} F_{2}\left(y+\frac{1}{2} h_{2}(y)\right)+\frac{1}{3 !} F_{3}\left(y+\frac{1}{2} h_{2}(y)\right)+\cdots+ \\
& +\frac{1}{(r-1) !} F_{r-1}\left(y+\frac{1}{2} h_{2}(y)\right)+O\left(|y|^{r}\right) .
\end{aligned}
$$

Soja $h_{j}(y)$ un função en $y$ de ordem $j, h_{j}(y) \in \mathbb{R}^{n}$, com $2 \leq j \leq r-1$; usando o binomio de Newton, obtemos:

$$
F_{k}\left(y+\frac{1}{2} h_{j}(y)\right)=F_{k}(y)+O\left(|y|^{k+(j-1)}\right)
$$

pala $2 \leq k \leq r-1$

Assim. para $j=2$ temos:

$$
\begin{aligned}
\left(I+\frac{1}{2} D h_{2}(y)\right) \dot{y}= & J y+\frac{1}{2} J h_{2}(y)+\frac{1}{2} F_{2}(y)+\frac{1}{3 !} F_{3}(y)+\cdots+ \\
& +\frac{1}{(r-1) !} \bar{F}_{r-1}(y)+O\left(|y|^{r}\right)
\end{aligned}
$$

onde $F(y) .3 \leq k: \leq r-1$, demotia os termos de ordem $k$ que tem sido modificados pela transformaçio de coordenadas.

Agora, para $y$ suficientemente pequeno, o operador

$$
\left(I+\frac{1}{2} D h_{2}(y)\right)^{\cdots 1}
$$


existe a premos representíl-lo em série de expansão como segue:

$$
\left(I+\frac{1}{2} D h_{2}(y)\right)^{-1} \ldots-I-\frac{1}{2} I h h_{2}(y)+O\left(\left.! y\right|^{2}\right)
$$

Observemos que:

$$
\left(I+\frac{1}{2} D h_{2}(y)\right)\left(I+\frac{1}{2} D h_{2}(y)\right)^{-1}(y)=y \Leftrightarrow-\frac{1}{4} D h_{2}(y) D h_{2}(y) y+O\left(|y|^{3}\right)=0
$$

on seja, os termos até ordem dois são completamente determinados.

Substituindo (2.7) em (2.6), obtemos:

$$
\dot{y}=J y+\frac{1}{2}\left[H_{2}(y)-\left(D h_{2}(y) J_{y}-J h_{2}(y)\right)\right]+\frac{1}{3 !} \vec{F}_{3}(y)+\cdots+\frac{1}{(r-1) !} \bar{F}_{r-1}^{r}(y)+O\left(|y|^{r}\right) .
$$

A té o momento, a função $h_{2}(y)$ foi tomada arbitrariamente. A fin de climinamos os termos de ordem dois da equação (2.8), a melhor escolha de $h_{2}(y)$ é aquela que satisfaz:

$$
D h_{2}(y) J y-J h_{2}(y)=F_{2}(y)
$$

Observemos une, com a escolha acima, eliminamos $F_{2}(y)$ de (2.8); mas infelizmente isso nem sempre ó possivel, sendo assim, escolheremos $h_{2}(y)$ de modo a simpliticar a e(puação $(2.8)$.

Para determinar a escolha adequada para $h_{2}(y)$, precisamos definir um operador linear sobre um espaço vetorial; isso será feito através de trếs etapas.

$1^{\circ}$ passo: Denotamos por $I_{2}^{n}$ o espaço vetorial dos polinônios homogeneos de gran 2 (ent $n$ variáveis reais.

Sejam $\left\{s_{1}, \ldots, s_{n}\right\}$ uma base de $\mathbb{R}^{n}$ e $y=\left(y_{1}, \ldots, y_{n}\right)$ as coordenadas de $y$ em relação a essa base. O conjunto

$$
\left\{\left(y_{1}^{m_{1}} y_{2}^{m_{2}} \ldots y_{n}^{n_{n}}\right) s_{i}, \quad i=1,2, \ldots, n, \quad \sum_{j=1}^{n} m_{j}=2, m_{j} \in \mathbb{N} e m_{j} \geq 0\right\}
$$

forma uma base para $H_{2}^{n}$.

$2^{\circ}$ passo: Definimos un operador lincar sobre $H_{2}^{n}$ :

$$
\begin{aligned}
& M_{2}: H_{2}^{n} \longrightarrow H_{2}^{n}, \\
& M_{2}\left(h_{2}(y)\right)=D h_{2}(y) J y-J h_{2}(y) .
\end{aligned}
$$

30passo: Consideramos a decomposição do espaço $H_{2}^{n}$ em luma soma direta dos espacos $G_{2}$ e $M_{2}\left(H_{2}^{n}\right)$, onde $G_{2}$ é o espaço complementiar de $M_{2}\left(H_{2}^{n}\right)$, on seja.

$$
H_{2}^{n}=M_{2}\left(H_{2}^{n}\right) \oplus G_{2}
$$

Assim, se $F_{2}$ estiver na imagem de $M_{2}$, podenos eliminar os termos de ordem dois la equação (2.8). Caso contrário, escolhemos $h_{2}(y)$ pertencente à $H_{2}^{n}$ tal que somente os temmos de ordem dois que estão em $G_{2}$, permanecem na equação (2.8). Denotanos os 
termos de ordem dois que permanecem na equação (2.8) por $F_{2}^{r}$, é claro que $F_{2}^{r} \in G_{2}$.

Portanto. podemos simplificar a equação (2.8) :

$$
\dot{y}=f y+\frac{1}{2} F_{2}^{r}(y)+\frac{1}{3 !} \bar{F}_{3}(y)+\cdots+\frac{1}{(r-1) !} F_{r-1}(y)+O\left(|y|^{r}\right)
$$

Aplicando cuse processo aos termos de ordem superior, obtemos o seguinte teorema:

Teorema 2.2.1 Por uma sucessiva mudança de coordenadas próximas à origem. transformamos a rquaçào $(2.1)$ em sua forma normal dada por:

$$
\dot{y}=J y+\frac{1}{2} F_{2}^{r}(y)++\frac{1}{3 !} F_{3}^{r}(y)+\cdots+\frac{1}{(r-1) !} F_{r-1}^{r}(y)+O\left(|y|^{r}\right),
$$

omle $F_{j}^{r}(y) \in G_{j}, 2 \leq j \leq(r-1)$ e $G_{j}$ é o espaço complementar de $M_{j}\left(H_{j}^{n}\right)$.

\section{Observaçōes:}

$1^{\circ}$ A estrutura dos termos não lineares em (2.9) é inteiramente determinada pela parte lincar da equação.

$2^{\circ}$ Ao simplificarmos os termos de ordem $k$, vemos, por (2.6), que os termos de ordem inferior nào mudam, mas os termos de ordem superior a $k$ são modificados.

$3^{\circ}$ Como a forma normal depende da escolha do espaço complementar $G_{k}$, temos que ela não é única.

O corolário que segue do próximo lema nos será muito ítil na determinaçào dos termos que permanecem na forma normal.

Lema 2.2.1 Consideremos o operador:

$$
\begin{aligned}
& M_{k}: H_{k}^{n} \longrightarrow H_{k}^{n}, \\
& M_{k}\left(h_{k}(y)\right)=D_{y} h_{k}(y) J y-J h_{k}(y),
\end{aligned}
$$

com $2 \leq k \leq r-1$

Se $\sigma(J)=\left\{\lambda_{1}, \lambda_{2}, \ldots, \lambda_{n}\right\}$, então:

$$
\sigma\left(M_{k}\right)=\left\{(q, \bar{\lambda})-\lambda_{j}: j=1,2, \ldots, n \text { e }|q|=k\right\},
$$

ondr: $q \in \mathbb{N}^{n}, q=\left(q_{1}, q_{2}, \ldots, q_{n}\right), \bar{\lambda}=\left(\lambda_{1}, \ldots, \lambda_{n}\right) e(q, \bar{\lambda})=q_{1} \lambda_{1}+q_{2} \lambda_{2}+\ldots+q_{n} \lambda_{n}$.

\section{Demonstração:}

Sabcmos que um número complexo $\Lambda$ pertence à $\sigma\left(M_{k}\right)$ se, e somente se, existe $h$ pertencente à $H_{k}^{n} \backslash\{0\}$ tal que $M_{k} h=\Lambda h$. Consideremos uma matriz não singular $S$, $n \times n$ e fazendo $y=S x$ e $g(x)=S^{-1} h(S x)$, obtemos:

$M_{k} h(y)=\frac{\partial h(y)}{\partial y} J y-J h(y)=S \frac{\partial g(x)}{\partial x} \frac{d x}{d y} J(S x)-J S g(x)=S \frac{\partial g(x)}{\partial x}\left(S^{-1} J S\right) x-J S g(x)$.

Portanto, definimos o operador $\bar{M}_{k}: H_{k}^{n} \rightarrow H_{k}^{n}$ por:

$$
\tilde{M}_{k} g(x) \stackrel{\text { def. }}{=} S^{-1} M_{k}(h(S x))=\frac{\partial g(x)}{\partial x}\left(S^{-1} J S\right) x-\left(S^{-1} J S\right) g(x) .
$$


()hservemes gue $M_{k} h=\Lambda h$ é equivalente à $M_{k} g=\Lambda g$.

D) fato:

Sir $M_{k} h=-\Lambda h$, nitio

$$
M_{k} g(x) \stackrel{\text { llof. }}{=} S^{-1} M_{k} h(S x)=S^{1} \Lambda h(S x)=\Lambda S^{-1} h(S x)=\Lambda g(x) .
$$

$S_{1} M_{k}: y=\Lambda g$, contion

$$
S M_{k} h(S x) \stackrel{\text { luff. }}{=} M_{k} g(x)=\Lambda g(x)=\Lambda S^{-1} h(S x)=S^{-1} \Lambda h(S x) \Rightarrow M_{k} h(S x)=\Lambda h(S x)
$$

Escolhenos $S$ de modo que $\left(S^{-1} J S\right)$ esteja na forma canonica do Jordan, com seus blocos formados por matrizes triangulares inferiores e denotemos os termos abaixo da diagonal por $\sigma_{i}, \quad i=2,3, \ldots, n$, on seja,

$$
S^{-1} J S=\left[\begin{array}{cccccc}
\lambda_{1} & 0 & 0 & 0 & \ldots & 0 \\
\sigma_{2} & \lambda_{2} & 0 & 0 & \ldots & 0 \\
0 & \sigma_{3} & \lambda_{3} & 0 & \ldots & 0 \\
\vdots & \vdots & \vdots & \vdots & \ddots & \vdots \\
0 & 0 & 0 & 0 & \ldots & \lambda_{n}
\end{array}\right] .
$$

Coloquemos $\sigma_{1}=0, \quad x_{0}=0, \quad g_{0}=0$ e $\bar{M}_{k: g}=\left(f_{1}, f_{2}, \ldots, f_{n}\right)=f$. Então:

$$
\begin{aligned}
& M_{k} g(x)=\left[\begin{array}{cccc}
\frac{\partial g_{1}(x)}{\partial x_{1}} & \frac{\partial g_{1}(x)}{\partial x_{2}} & \ldots & \frac{\partial g_{1}(x)}{\partial x_{n}} \\
\frac{\partial g_{2}(x)}{\partial x_{1}} & \frac{\partial g_{2}(x)}{\partial x_{2}} & \ldots & \frac{\partial g_{2}(x)}{\partial x_{n}} \\
\vdots & \vdots & \ddots & \vdots \\
\frac{\partial g_{n}(x)}{\partial x_{1}} & \frac{\partial g_{n}(x)}{\partial x_{2}} & \ldots & \frac{\partial g_{n}(x)}{\partial x_{n}}
\end{array}\right] \cdot\left[\begin{array}{cccccc}
\lambda_{1} & 0 & 0 & 0 & \ldots & 0 \\
\sigma_{2} & \lambda_{2} & 0 & 0 & \ldots & 0 \\
0 & \sigma_{3} & \lambda_{3} & 0 & \ldots & 0 \\
\vdots & \vdots & \vdots & \vdots & \ddots & \vdots \\
0 & 0 & 0 & 0 & \ldots & \lambda_{n}
\end{array}\right] \cdot\left[\begin{array}{c}
x_{1} \\
x_{2} \\
\vdots \\
x_{n}
\end{array}\right]- \\
& -\left[\begin{array}{cccccc}
\lambda_{1} & 0 & 0 & 0 & \ldots & 0 \\
\sigma_{2} & \lambda_{2} & 0 & 0 & \ldots & 0 \\
0 & \sigma_{3} & \lambda_{3} & 0 & \ldots & 0 \\
\vdots & \vdots & \vdots & \vdots & \ddots & \vdots \\
0 & 0 & 0 & 0 & \ldots & \lambda_{n}
\end{array}\right] \cdot\left[\begin{array}{c}
g_{1}(x) \\
g_{2}(x) \\
\vdots \\
g_{n}(x)
\end{array}\right]
\end{aligned}
$$




$$
=\left[\begin{array}{c}
\frac{\partial g_{n}(x)}{\partial r_{1}} \lambda_{1} \cdot x_{1}+\frac{\partial g_{1}(x)}{\partial x_{2}}\left(\sigma_{2} \cdot x_{1}+\lambda_{2} x_{2}\right)+\cdots+\frac{\partial g_{1}(x)}{\partial x_{n}}\left(\lambda_{n} x_{n}\right)-\lambda_{1} g_{1}(x) \\
\frac{\partial g_{2}(x)}{\partial x_{1}} \lambda_{1} x_{1}+\frac{\partial g_{2}(x)}{\partial x_{2}}\left(\sigma_{2} x_{1}+\lambda_{2} x_{2}\right)+\cdots+\frac{\partial g_{2}(x)}{\partial x_{n}}\left(\lambda_{n} \cdot x_{n}\right)-\lambda_{2} g_{2}(x)-\sigma_{2} \cdot g_{1}(x) \\
\vdots \\
\frac{\partial g_{n}(x)}{\partial x_{1}} \lambda_{1} \cdot x_{1}+\frac{\partial g_{n}(x)}{\partial x_{2}}\left(\sigma_{2} \cdot x_{1}+\lambda_{2} \cdot x_{2}\right)+\cdots+\frac{\partial g_{n}(x)}{\partial x_{n}}\left(\lambda_{n} x_{n}\right)-\lambda_{n} g_{n}(x)-\sigma_{n} \cdot g_{n-1}(x)
\end{array}\right] .
$$

Portanto, para cada $j=1,2, \ldots, n$, temos:

$$
f_{j}(x)=\sum_{i=1}^{n} \frac{\partial g_{j}(x)}{\partial x_{i}}\left(\lambda_{i} x_{i}+\sigma_{i} x_{i-1}\right)-\lambda_{j} g_{j}(x)-\sigma_{j} g_{j-1}(x)
$$

como $g(x)$ e $f(x)$ pertencem à $H_{k}^{n}$, segue que $g_{j}(x)$ e $f_{j}(x)$ pertencem à $H_{k}^{1}$, ou seja,

$$
g_{j}(x)=\sum_{|q|=k} g_{j}^{(q)} x^{q} \text { e } f_{j}(x)=\sum_{|q|=k} f_{j}^{(q)} x^{q}
$$

Agora. determinemos os coeficientes $f_{j}^{(q)}$ para cada $j=1,2, \ldots, n$.

$$
\begin{aligned}
& f_{j}(x)=\sum_{i=1}^{n} \frac{\partial\left(\sum_{i q \mid=k} g_{j}^{(q)} x^{q}\right)}{\partial x_{i}}\left(\lambda_{i} x_{i}+\sigma_{i} x_{i-1}\right)-\lambda_{j} \sum_{|q|=k} g_{j}^{(q)} x^{q}-\sigma_{j} \sum_{|q|=k} g_{j-1}^{(q)} x^{q} \\
& =\sum_{i=1}^{n} \sum_{|q|=k} g_{j}^{(q)} q_{i} x^{\left(q-e_{i}\right)}\left(\lambda_{i} x_{i}+\sigma_{i} x_{i-1}\right)-\sum_{|q|=k} \lambda_{j} g_{j}^{(q)} x^{q}-\sum_{|q|=k} \sigma_{j} g_{j-1}^{(q)} x^{q} \\
& =\sum_{|q|=k}\left[\sum_{i=1}^{n}\left(g_{j}^{(g)} \lambda_{i} q_{i} x^{q}+g_{j}^{(q)} \sigma_{i} q_{i} x^{q+e_{i-1}-e_{i}}\right)-\lambda_{j} g_{j}^{(q)} x^{q}-\sigma_{j} g_{j-1}^{(q)} x^{q}\right] \\
& =\sum_{|q|=k}\left[\sum_{i=1}^{n}\left(\lambda_{i} q_{i}-\lambda_{j}\right) g_{j}^{(q)} x^{g}+\sum_{i=1}^{n} g_{j}^{(q)} \sigma_{i} q_{i} x^{q+c_{i-1}-c_{i}}-\sigma_{j} g_{j-1}^{(q)} x^{q}\right] \\
& =\sum_{|q|=k}\left[\left[(q, \lambda)-\lambda_{j}\right] g_{j}^{(q)}-\sigma_{j} g_{j-1}^{(q)}\right] x^{q}+\sum_{i=2}^{n} \sum_{|q| \mid=k} g_{j}^{(q)} \sigma_{i} q_{i} x^{q+e_{i-1}-e_{i}} \\
& =\sum_{|q|=k}\left[\left[(q, \bar{\lambda})-\lambda_{j}\right] g_{j}^{(q)}-\sigma_{j} g_{j-1}^{(q)}\right] x^{q}+\sum_{i=2}^{n} \sum_{\substack{\left|q^{\prime}\right|=k \\
q^{\prime} \neq q^{-}-e_{i-1}+e_{i}}} g_{j}^{\left(q^{\prime}\right)} \sigma_{i} q_{i} x^{q^{\prime}+e_{i-1}-c_{i}}+
\end{aligned}
$$




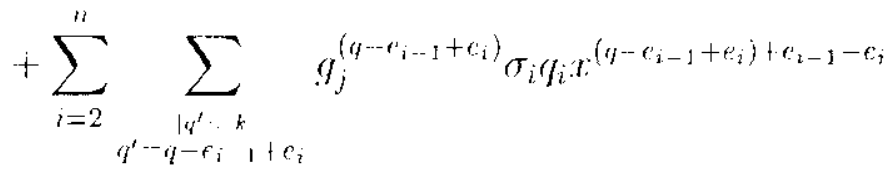

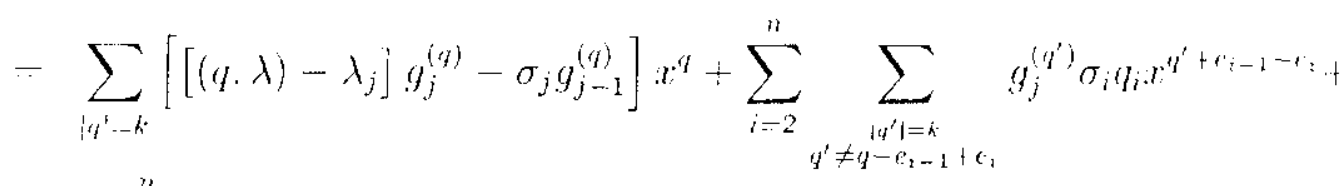

$$
\begin{aligned}
& +\sum_{i=2}^{\prime \prime} \sum_{|q|=k} g_{j}^{\left(i\left(i_{i}-1+c_{2}\right)\right.} \sigma_{i} q_{i} x^{\prime \prime} \\
& =\sum_{|q| \cdots k}\left[\left[(q, \bar{\lambda})-\lambda_{j}\right] g_{j}^{(q)}+\sum_{i=2}^{n} q_{i} \sigma_{i} g_{j}^{\left(q-e_{i-1}+e_{i}\right)}-\sigma_{j} g_{j-1}^{(q)}\right] x^{q}+ \\
& +\sum_{i=2}^{n} \sum_{\substack{\mid q^{\prime}=k \\
q^{\prime} \neq q^{\prime}=e_{i-1}+e_{i}}} ! !_{j}^{\left(q^{\prime}\right)} \sigma_{i} q_{i} x^{q^{\prime}+e_{i-1}-e_{i}}
\end{aligned}
$$

Portanto. obtenos:

$$
f_{j}^{(q)}=\left[(q, \bar{\lambda})-\lambda_{j}\right] g_{j}^{(q)}+\sum_{i=2}^{n} q_{i} \sigma_{i} g_{j}^{\left.(q) \cdot c_{i-1}+r_{i}\right)}-\sigma_{j} g_{j-1}^{(q)},
$$

onde $e_{i}=\left(\delta_{1, i}, \delta_{2, i}, \ldots, \delta_{n, i}\right), \quad \delta_{k, i}=1$ se $k=i$ e $\delta_{k, i}=0$ se $k \neq i$.

Seja $v \in H_{k}^{n}$, entäo podemos escrever:

$$
v(x)=\sum_{|q|=k} v^{(q)} x_{1}^{g_{1}} x_{2}^{q_{2}} \ldots x_{n}^{q_{n}},
$$

onde $v^{q}=\left\langle v_{1}^{(q)}, v_{2}^{(q)}, \ldots, v_{n}^{(q)}\right)$. Dizemos que $v_{k}^{(q)}$ precede $v_{f}^{(p)}$ se a primeira diferença não nula $l-k, p_{1}-q_{1}, \ldots, p_{n}-q_{n}$ é positiva.

Desse modo, temos que $g_{j}^{(q)}$ é precedido por $g_{j}^{\left(q-e_{i-1}+e_{i}\right)}$ e $g_{j-1}^{(q)}$

Entào, conclúmos:

$\Lambda \in \sigma\left(M_{k}\right)$ se, e somente se, $\Lambda \in \sigma\left(\bar{M}_{k}\right)$ o que equivale a existir $g(x)$ pertencente à $H_{k}^{\prime \prime} \backslash\{0\}$ tal que

$$
\bar{M}_{k} g(x)=\Lambda g(x)
$$

ou seja,

$$
\bar{M}_{k} g_{j}(x)=\Lambda g_{j}(x)
$$

Mas como

$$
\vec{M}_{k} g_{j}(x)=f_{j}(x)=\sum_{|q|=k} f_{j}^{(q)} x^{q}
$$

onde $f_{j}^{(q)}$ é dado acima e $g_{j}(x)=\sum_{|q|=k} g_{j}^{(q)} x^{q}$, temos que:

$$
\left[(q, \bar{\lambda})-\lambda_{j}\right] g_{j}^{(q)}+\sum_{i=2}^{n} q_{i} \sigma_{i} g_{j}^{\left(q-e_{i-1}+e_{i}\right)}-\sigma_{j} g_{j-1}^{(q)}=\Lambda g_{j}^{(q)} .
$$




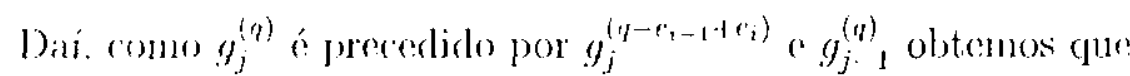

$$
\Lambda=\left[(q, \lambda)-\lambda_{j}\right]
$$

para algum qo $\lambda_{j}$.

Corolário 2.2.1 : Seja $k$ tal que $2 \leq k \leq r-1$. Se todo elemento $A$ pertencente à $\sigma\left(M_{k}\right)$ for mion mulo. entaio podemos climinar os termos de ordem $k$ a da forma normal.

\section{Demonstração:}

Como poekenos escrever o espaço $I_{k}^{n}$ cono soma direta da imagem e do núcleo do operador $M_{k}$, ó suficiente verificarmos que o núcleo do operador $M_{k}$ contém apenas o polinomio identicamente nulo.

Suponlamos que existe $g(x) \in \operatorname{Ker}\left(M_{k}\right) \backslash\{0\}$, então temos que:

$$
M_{k} g(x) \equiv 0
$$

o que implica que 0 pertence ao espectro de $M_{k}$.

\subsection{Exemplos}

Exemplo 2.3.1 : Consideremos a equação:

$$
\dot{x}=J x+F(x), \quad x \in \mathbb{R}^{n},
$$

onde $J \equiv 0 \circ F^{\prime}: \mathbb{R}^{n} \rightarrow \mathbb{R}^{n}$ é náo linear em $x$, de classe $C^{r}$.

Dai. temos que o operador:

$$
\begin{aligned}
& M_{k}: H_{k}^{n} \longrightarrow H_{k}^{n}, \\
& M_{k}\left(h_{k}(x)\right)=D h_{k}(x) J x-J h_{k}(x)
\end{aligned}
$$

6 identicamente nulo.

Logo, a imagem de $M_{k}$ é $\{0\}$.

Portanto, o espaço complementar $G_{k}$ de $M_{k}\left(H_{k}^{n}\right)$ é todo o espaço $H_{k}^{n}$, para todo $k ;$ o que implica que os termos não lineares permanecem na forma normal.

Exemplo 2.3.2 : Consideremos a equação:

$$
\dot{x}=J x+F(x), \quad x=\left(x_{1}, x_{2}\right)^{T} \in \mathbb{R}^{2},
$$

onde $J=\operatorname{diag}(1,3)$ e $F: \mathbb{R}^{2} \rightarrow \mathbb{R}^{2}$ é näo linear em $x$, de classe $C^{r}, r \geq 3$.

Para $k \geq 2$ e $j \in \mathbb{N}$, tomemos $q=(k-j, j)$ pertencente à $\mathbb{N}^{2}, \ddot{\lambda}=(1,3), \lambda_{1}=1$ e $\lambda_{2}=3$, entaio temos $|q|=k$, e ainda

$$
(q, \bar{\lambda})-\lambda_{i}=0, \quad i=1,2
$$


se. r somentess

$$
\begin{aligned}
& k+2 j=1, \text { se } i=1, \\
& k+2 j=3, \text { se } i=2 .
\end{aligned}
$$

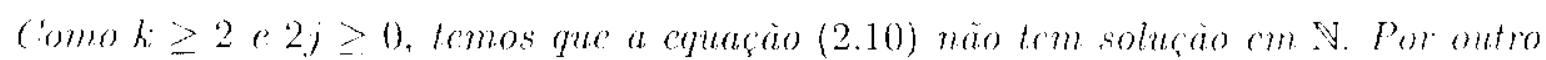
lado. trmos que a rquaço (2.11) está satisfeita se e somente se $k=3$ e $j=0$. on soja. 1] $-(3,0)$.

Pomlamo, do Conolírio 2.2.1, podemos eliminar os termos de ordem ki da forma normal. jura todo $2 \leq k \leq r-1$ ek:3.

Sabemos que o operador

$$
M_{3}: H_{3}^{2} \rightarrow H_{3}^{2}
$$

i dato por:

$$
M_{3}(h(x))=\left[\begin{array}{c}
x_{1} \frac{\partial h_{1}(x)}{\partial x_{1}}+3 x_{2} \frac{\partial h_{1}(x)}{\partial x_{2}}-h_{1}(x) \\
x_{1} \frac{\partial h_{2}(x)}{\partial x_{1}}+3 x_{2} \frac{\partial h_{2}(x)}{\partial x_{2}}-3 h_{2}(x)
\end{array}\right],
$$

omder: $x:=\left(x_{1}, x_{2}\right)^{T}$ e $h(x)=\left(h_{1}(x), h_{2}(x)\right)^{T}$.

Se considerarmos a base canônica de $I_{3}^{2}$,

$$
\left(\begin{array}{c}
x_{1}^{3} \\
0
\end{array}\right),\left(\begin{array}{c}
x_{1}^{2} x_{2} \\
0
\end{array}\right),\left(\begin{array}{c}
x_{1} x_{2}^{2} \\
0
\end{array}\right),\left(\begin{array}{c}
x_{2}^{3} \\
0
\end{array}\right),\left(\begin{array}{c}
0 \\
x_{1}^{3}
\end{array}\right),\left(\begin{array}{c}
0 \\
x_{1}^{2} x_{2}
\end{array}\right),\left(\begin{array}{c}
0 \\
x_{1} x_{2}^{2}
\end{array}\right),\left(\begin{array}{c}
0 \\
x_{2}^{3}
\end{array}\right),
$$

cminu, suas respectivas imagens, sob o operador $M_{3}$, sào:

$$
\left(\begin{array}{c}
2 x_{1}^{3} \\
0
\end{array}\right),\left(\begin{array}{c}
4 x_{1}^{2} x_{2} \\
0
\end{array}\right),\left(\begin{array}{c}
6 x_{1} x_{2}^{2} \\
0
\end{array}\right),\left(\begin{array}{c}
8 x_{2}^{3} \\
0
\end{array}\right),\left(\begin{array}{l}
0 \\
0
\end{array}\right),\left(\begin{array}{c}
0 \\
2 x_{1}^{2} x_{2}
\end{array}\right),\left(\begin{array}{c}
0 \\
4 x_{1} x_{2}^{2}
\end{array}\right),\left(\begin{array}{c}
0 \\
6 x_{2}^{3}
\end{array}\right) .
$$

Portanto, somente os termos que pertencem ao espaço vetorial gerado por $\left(\begin{array}{c}0 \\ x_{1}^{3}\end{array}\right)$, nio podem ser eliminados da forma normal.

Assim. a forma normal é dada por:

$$
\left\{\begin{array}{l}
\dot{x}_{1}=x_{1} \\
\dot{x}_{2}=3 x_{2}+c x_{1}^{3}
\end{array}\right.
$$

onde' cé uma constante real.

Exemplo 2.3.3 : Consideremos a cquaçäo:

$$
\dot{x}=J x+F(x), \quad x \in \mathbb{R}^{2} .
$$

onde $J=\left[\begin{array}{ll}0 & 1 \\ 0 & 0\end{array}\right]$ e $F: \mathbb{R}^{2} \longrightarrow \mathbb{R}^{2}$ é não linear em $x$, de classe $C^{r}$.

() operador

$$
M_{2}: H_{2}^{2} \rightarrow I_{2}^{2}
$$


stato per:

$$
M_{2}(h(x))=\left[\begin{array}{l}
x_{2} \frac{\partial h_{1}(x)}{\partial x_{1}}-h_{2}(x) \\
x_{2} \frac{\partial h_{2}(x)}{\partial x_{1}}
\end{array}\right]
$$

$\left(r m, r \cdots\left(r_{1}, x_{2}\right)^{T}\right.$ eh $(x)-\left(h_{1}(x), h_{2}(x)\right)^{T}$.

se romsidemmos a base canomica de $\mathrm{H}_{2}^{2}$,

$$
\left(\begin{array}{c}
x_{1}^{2} \\
0
\end{array}\right),\left(\begin{array}{c}
x_{1} x_{2} \\
0
\end{array}\right),\left(\begin{array}{c}
x_{2}^{2} \\
0
\end{array}\right),\left(\begin{array}{c}
0 \\
x_{1}^{2}
\end{array}\right),\left(\begin{array}{c}
0 \\
x_{1} x_{2}
\end{array}\right) \cdot\left(\begin{array}{c}
0 \\
x_{2}^{2}
\end{array}\right),
$$

entio, suas respectivas imagens, sob) o operador $M_{2}$, săo:

$$
\left(\begin{array}{c}
2 x_{1} x_{2} \\
0
\end{array}\right):\left(\begin{array}{c}
x_{2}^{2} \\
0
\end{array}\right),\left(\begin{array}{l}
0 \\
0
\end{array}\right),\left(\begin{array}{c}
-x_{1}^{2} \\
2 x_{1} x_{2}
\end{array}\right),\left(\begin{array}{c}
-x_{1} x_{2} \\
x_{2}^{2}
\end{array}\right),\left(\begin{array}{c}
-x_{2}^{2} \\
0
\end{array}\right) .
$$

Portanto, temos que:

$$
M_{2}\left(H_{2}^{2}\right)=\operatorname{gcr}\left\{\left(\begin{array}{c}
2 x_{1} x_{2} \\
0
\end{array}\right),\left(\begin{array}{c}
x_{2}^{2} \\
0
\end{array}\right),\left(\begin{array}{c}
-x_{1}^{2} \\
2 x_{1} x_{2}
\end{array}\right),\left(\begin{array}{c}
-x_{1} x_{2} \\
x_{2}^{2}
\end{array}\right)\right\} .
$$

Daí como os vetores

$$
\left(\begin{array}{c}
2 x_{1} x_{2} \\
0
\end{array}\right),\left(\begin{array}{c}
x_{2}^{2} \\
0
\end{array}\right),\left(\begin{array}{c}
-x_{1}^{2} \\
2 x_{1} x_{2}
\end{array}\right),\left(\begin{array}{c}
-x_{1} x_{2} \\
x_{2}^{2}
\end{array}\right),\left(\begin{array}{c}
x_{1}^{2} \\
\frac{1}{2} x_{1} x_{2}
\end{array}\right),\left(\begin{array}{c}
0 \\
x_{1}^{2}
\end{array}\right),
$$

sion linearmente independentes, podemos escolher o espaço complementar de $M_{2}\left(H_{2}^{2}\right)$, romo:

$$
G_{2}=\operatorname{ger}\left\{\left(\begin{array}{c}
x_{1}^{2} \\
\frac{1}{2} x_{1} x_{2}
\end{array}\right),\left(\begin{array}{c}
0 \\
x_{1}^{2}
\end{array}\right)\right\} \text {. }
$$

Assim. a forma normal, até os termos de segunda ordem, é dada por:

$$
\left\{\begin{array}{l}
\dot{x}_{1}=x_{2}+a x_{1}^{2}+O\left(|x|^{3}\right) \\
\dot{x}_{2}=b x_{1}^{2}+c x_{1} x_{2}+O\left(|x|^{3}\right)
\end{array}\right.
$$

onde a,b e ciro constantes rears.

Podemos ainda tomar o espaço complcmentar $G_{2}$ como:

$$
G_{2}=\operatorname{ger}\left\{\left(\begin{array}{c}
0 \\
x_{1}^{2}
\end{array}\right),\left(\begin{array}{c}
0 \\
x_{1} x_{2}
\end{array}\right)\right\} \text {. }
$$

Desse modo, a forma normal, até os termos de segunda ondem, é dada por:

$$
\left\{\begin{array}{l}
\dot{x}_{1}=x_{2}+O\left(|x|^{3}\right) \\
\dot{x}_{2}=a x_{1}^{2}+b x_{1} x_{2}+O\left(|x|^{3}\right)
\end{array}\right.
$$

onde a c b sio constantes reais. 


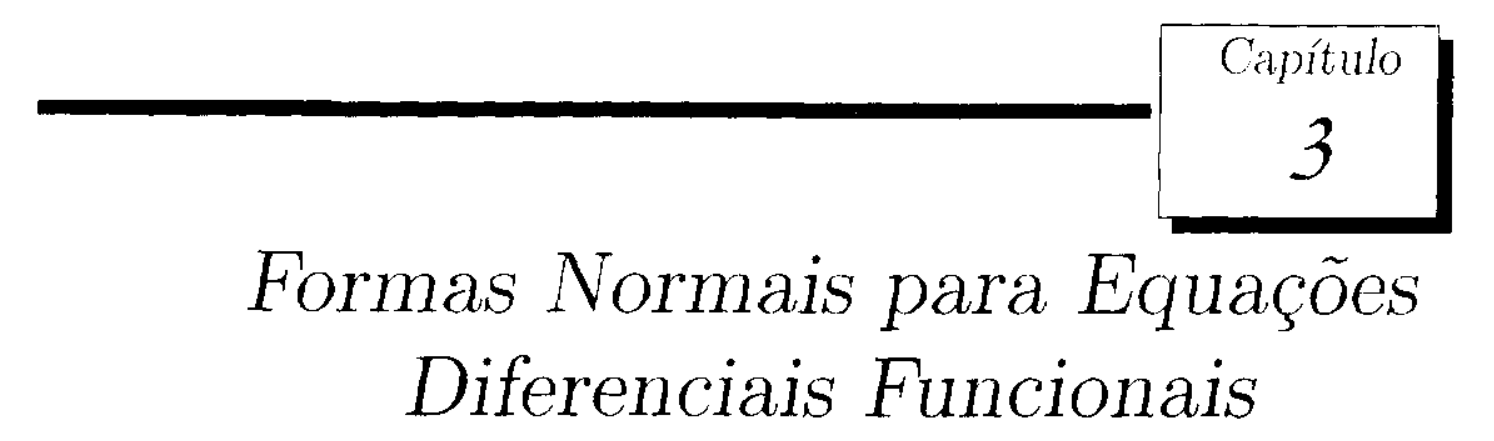

\subsection{Introdução}

Neste capítulo, obteremos o método da forma normal para equaçōes diferenciais funcionais retardatlas. Para fazermos isso, será necessário consideramos uma equação diferencial funcional como uma expração diforencial ordinária abstrata en um espaco de fase de dimensão infinitia.

O método da forma normal nos dará o fluxo da equação diferencial funcional retardada (mu unn espaço invariante de dimensão finita associado a un conjunto finito de antovalores do gerador infinitesimal da equação linearizada na singularidade. Para isso, decompomos o cspaço de fase como soma direta de um espaço invariante para o gerador infinitesimal da equação linearizada e sen espaço complementar, também invariante. Fssa decomposição será feita aplicando a teoria desenvolvida no Capítulo 1. O método da forma normal depende de algumas condiçoes denominadas de não-ressonância, que desenvolveremos na Seção 3.4. Apesar do uso de série de potências para a parte não-linear da equação diferencial funcional retardada original e para as mudanças de variáveis, não nos preocuparemos com a convergência dessas séries, uma ver que, estarenos interessados em obter a forma normal até uma certa ordom finita. Como em muitos casos é conveniente introduzirmos 1 marâmetro para que possamos descrever completamente a singularidade, também desenvolveremos a forma normal para equações diferenciais funcionais retardadas com um parâmetro.

Por último, faremos aplicaços à singularidade do tipo Bogdanov-Takens. (O nosso estudo consistirá, numa situação geral, do cálculo da forma normal para equações diferenriais funcionais retardadas escalares com una singularidade do tipo Bogdanov-'Takens.

\subsection{Equações Diferenciais Funcionais}

Considerenos $T^{\prime}(t), t \geq 0$ o operador solução do sistema de equações diferenciais ordinárias:

$$
\dot{x}=A x,
$$




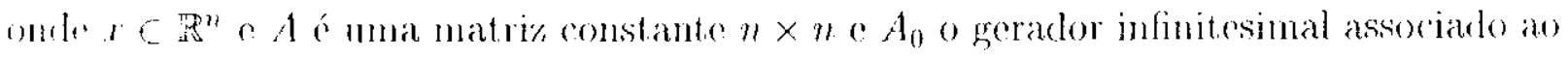
Cir-semigrupo $T($ l) dado por:

$$
\begin{gathered}
A_{0}: D\left(A_{0}\right) \longrightarrow \mathbb{R}^{n}, \\
x \mapsto A x .
\end{gathered}
$$

('omo vimos, no capitulo anterior, a matris $A$ é de fundamental inportancia para "resenvolvinento da forma nomal da equaçio diferencial ordinária, e por isso. introluziremos ats comdiçoes necessárias para escrever mma equaçăo diferencial funcional como mma equaço diferencial ordinária abstrata $\mathrm{em}$ mm espaço de Banach adequado, onde a decomposição dos termos lineares e não lincares da equaçäo está bem formulada. Ėm seguida. usaremos a teoria de semigrupos para decompor essa equação diferencial ordinária abstratia em duas equaçōes, sendo uma de dimensão linita e outra de dinensão infinita.

Consideremos uma equação diferencial funcional retardada da forma:

$$
\dot{z}(t)=L\left(z_{t}\right)+F\left(z_{t}\right)
$$

onde $L$ é um operador linear limitado de $C=C\left([-r, 0], \mathbb{R}^{n}\right) \mathrm{cm} \mathbb{R}^{n}, F$ é uma funçào de classe $C^{N}, N \geq 2$, definida em $C=C\left([-r, 0], \mathbb{R}^{n}\right)$ com valores en $\mathbb{R}^{n}, F(0)=0$ 0 $D F(0)=0$.

A parte linear da equą̧ão diferencial funcional retardada (3.1) é dada por:

$$
\dot{z}(t)=L\left(z_{t}\right)
$$

() conjunto das soluções da equação (3.2) define um $C_{0}$-semigrupo sobre $C$, que denotamos por $T(t), t \geq 0$. Assim, temos que o gerador infinitesimal associado ao $C_{0}$-semigrupo $T(t)$, dado no Lema 1.3.2, é definido por:

$$
\begin{aligned}
& A_{0}: D\left(A_{0}\right) \rightarrow C, \\
& A_{0} \phi=\dot{\phi},
\end{aligned}
$$

onde $D\left(A_{0}\right)=\left\{\phi \in C^{1}=C^{1}\left([-r, 0], \mathbb{R}^{n}\right): L(\phi)=\dot{\phi}(0)\right\}$.

Sabemos do Lema 1.4.1, que o espectro $\sigma\left(A_{0}\right)$ do gerador infinitesimal $A_{0}$ coincide com o espectro pontual $\sigma_{p}\left(A_{0}\right)$, e $\lambda \in \sigma\left(A_{0}\right)$ se, e somente se,

$$
\operatorname{det}(\Delta(\lambda))=0
$$

(.)III

$$
\Delta(\lambda)=\lambda I-\int_{-r}^{0}[d \eta(\theta)] e^{\lambda \theta}
$$

onder $\eta$ ć a matriz $n \times n$ cujos clementos são funçoes de variaçào limitada, definida sobre $[-r, 0]$, normalizada a fion de que $\eta$ scja contínua à esquerda sobre $(-r, 0)$, tal que:

$$
L(\phi)=\int_{-r}^{0}[d \eta(\theta)] \phi(\theta), \phi \in C .
$$

Os elementos do espectro pontual são ditos autovalores. 
Ioderíanos considerar a equaçio (3.1) como 11md equação diferencial ordinária abstratia m $C$.

$$
\frac{d u}{d t}=C(u)
$$

intile

$$
G(u)(\theta)=\left\{\begin{array}{l}
L(u)+F(u), \text { se } \theta=0 \\
u(\theta), \text { se } \theta \in[-r, 0] .
\end{array}\right.
$$

" "lomínio do $G \Leftrightarrow\left\{u \in C^{1}: \dot{u}(0)=L(u)+F(u)\right\}$. Mas, nesse espaço os temos lineares ? nào lineares nào podem ser adequadamente separados, pois a parte linear

$$
A_{0} u(\theta)=\left\{\begin{array}{l}
L(u), \text { se } \theta=0 \\
\dot{u}(\theta), \text { se } \theta \in[-r, 0),
\end{array}\right.
$$

1em domínio $\left\{u \in C^{1}: \dot{u}(0)=L(u)\right\}$, o qual seria compatível com o domínio de $G$ se, $e$ somente, se, $F(u)=0$.

I'ortanto, para que possamos ter a decomposição dos temos lineares e não lineares bem formulada, precisaremos estender o espaço $C$.

Defininos o conjunto $B C$ como sendo o espaço das finģos de $[-r, 0]$ em in". mifommente contínuas sobre $[-r, 0)$ e com possível descontimuidade no zero. Os elementos do $B C$ são da forma $\psi=\phi+X_{0} \alpha$, onde $\phi \in C, \alpha \in \mathbb{R}^{n}$ o $X_{0}$ é uma função definida por:

$$
X_{0}(\theta)= \begin{cases}I, & \text { se } \theta=0 \\ 0, & \text { se } \theta \neq 0 .\end{cases}
$$

tenos também, que esse espaço é identificado $\operatorname{com} C \times \mathbb{R}^{n}$. Com a norma:

$$
\left|\phi+X_{0} \alpha\right|=|\phi|_{C}+|\alpha|_{R^{n}}
$$

$B C$ é um espaço de Banach.

No espaço de fase $B C$, podemos escrever a equação diferencial ordinária abstrata associada à equação diferencial funcional (3.1), com a parte linear separada da parte näo lincar:

$$
\frac{d u}{d l}=A u+X_{0} F(u),
$$

ondo

$$
A \phi=\dot{\phi}+X_{0}[L(\phi)-\dot{\phi}(0)]
$$

r com domínio $D(A)=C^{\mathrm{i}}$.

Observemos que, para $\phi \in D\left(A_{0}\right)=\left\{\phi \in C^{1}=C^{1}\left([-r, 0], \mathbb{R}^{n}\right): L(\phi)=\dot{\phi}(0)\right\}$.

$$
A_{0} \phi=A \phi
$$

Se restringirmos o fluxo à variedade central associada à essa equação, podemos descrever completamente o comportannento das soluçōes da equação (3.1) em $C$ próximo à uma singularidade. Desse modo, precisamos considerar a forma normal relativa ao espaço $P$ invariante por $A_{0}$, o qual está associado com o conjunto não vazio e finito de autovalores 
$A-\left\{\lambda \in \sigma\left(A_{11}\right): R e(\lambda)=0\right\}$. De mancima majs geral, consideramemos a forma normal mativa a $11 \mathrm{~m}$ espaco $P$ invariante por $A_{0}$, associado a $u$ subconjunto finito e nao vazio doresentro do gerador infinitesimal $A_{0}$

Consideremos $A$ um subconjunto finito e não vazio do espectro do getartor infinitesinal $f_{11}$. tomemos a decomposiçáo de $C$ jor $\Lambda$, como no Teorma 1.4.1,

$$
C=P \oplus Q
$$

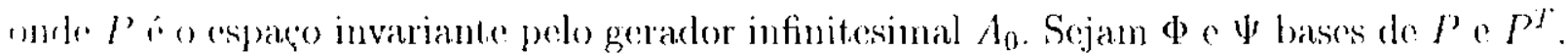
respertivamente, $\operatorname{com}(\Psi, \Phi)=I$, onde, $\left(.\right.$, . é a forma bilinear definida sobre $C^{*} \times C$.

$$
(\psi \cdot \phi)=\psi(0) \phi(0)-\int_{-r}^{0} \int_{0}^{\theta} \psi(\theta-\xi)[d \eta(\theta)] \phi(\xi) d \xi, \dot{\psi} \in C^{*}, \phi \in C
$$

Seja $m$ o número de elementos de $\Lambda$ contando suas multiplicidades, então segue do Loma 1.5 .4 que a dimensão de $P$ é $m$. Tomemos $B$ a matriz constante $m \times m$ tal que:

$$
\begin{aligned}
& A_{0} \Phi=\Phi B, \\
& A_{0}^{T} \Psi=B \Psi .
\end{aligned}
$$

Podemos estender nat.uralmente a forma bilinear definida sobre $C^{*} \times C$, ao espaço $C^{*} \times B C$ da seguinte maneira:

$$
\left(\Psi b, \phi+X_{0} \alpha\right)=(\Psi b, \phi)+\Psi(0) b \alpha ;
$$

também, a projeção de $C$ sobre $P$, dada por $\phi \rightarrow \Phi(\Psi, \phi)$, pode ser estendida por:

$$
\begin{aligned}
& \pi: B C \longrightarrow P \\
& \pi\left(\phi+X_{0} \alpha\right)=\Phi[(\Psi, \phi)+\Psi(0) \alpha]
\end{aligned}
$$

Portanto, podemos decompor $B C$ da forma:

$$
B C=P \oplus \operatorname{Ker}(\pi)
$$

é claro que $Q \varsubsetneqq \operatorname{Ker}(\pi) . \Lambda$ decomposição da função $u \in D(A)=C^{1}$ é:

$$
u=\Phi x+y,
$$

ande $x \in \mathbb{R}^{m} \quad 0 y \in \operatorname{Ker}(\pi) \cap C^{1}:=Q^{1}$.

sondo assim, consegnimos:

$$
\frac{d u}{d t}=\Phi \dot{x}+\frac{d y}{d t}
$$

Por outro lado,

$$
\frac{d u}{d t}=A(\Phi x+y)+X_{0} F(\Phi x+y)
$$


lisinde a projeção $\pi$, obtremos:

$$
\pi\left(\begin{array}{c}
d u \\
-d t
\end{array}\right)=\pi\left(\Phi x+\frac{d y}{d t}\right)=\pi\left(A(\Phi x+y)+X_{0} k(\Phi x+y)\right)
$$

(11) sieja,

$$
\Phi \dot{x}=\pi A(\Phi x+y)+\pi X_{0} F^{\prime}(\Phi x+y)=\pi A(\Phi x+y)+\Phi \psi(0) F(\Phi x+y) .
$$

Como A comnta com $\pi$ en $C^{1}$, temos:

$$
\Phi x=A \pi(\Phi x+y)+\Phi \Psi(0) F(\Phi x+y)=A \Phi x+\Phi \Psi(0) F(\Phi x+y) .
$$

Sendo $A \phi=A_{01} \phi$, para $\phi \in D\left(A_{0}\right)$ e por (3.6), conseguimos,

$$
\Phi x=A_{0} \Phi x+\Phi \Psi(0) F^{\prime}(\Phi x+y)=\Phi B x+\Phi \Psi(0) F^{\prime}(\Phi x+y),
$$

e portante.

$$
\dot{x}=B x+\Psi(0) F(\Phi x+y)
$$

Do mesmo nodo,

$$
(I-\pi)\left(\frac{d u}{d t}\right)=(I-\pi)\left(\Phi \dot{x}+\frac{d y}{d t}\right)=(I-\pi)\left(A(\Phi x+y)+X_{0} F(\Phi x+y)\right),
$$

ous seja,

$$
\frac{d y}{d t}==A_{Q^{\prime}} y+(I-\pi) X_{0} F(\Phi x+y),
$$

onde $A_{Q^{1}}$ denotia a restriçào de $A$ à $Q^{1}$.

Conclúmos assim, que a equação (3.4) é equivalente ao sistema:

$$
\left\{\begin{array}{l}
\dot{x}=B x+\Psi(0) F^{\prime}(\Phi x+y) \\
\frac{d y}{d t}=A_{Q^{1}} y+(I-\pi) X_{0} F(\Phi x+y),
\end{array}\right.
$$

com $x \in \mathbb{R}^{m}$ e $y \in Q^{1}=Q \cap C^{1}$.

\subsection{Formas Normais}

Nesta seção, obteremos a forma normal da equação (3.1), sobre um espaço invariante pelo gerador infinitesimal $A_{0}$ associado a um subconjunto finito e não vazio do espectro do $A_{0}$, de modo análogo ao procedimento que desenvolvemos para equaçós diferenciais ordinárias. Fstamos interessados on mudanças de variáveis para a equação (3.1) tal que $y=0$ defina uma variedade localmente invariante de dimensão finita sobre a qual o fluxo Ć dado por und equação diferencial ordiúria en sua forma normal $\mathrm{en} \mathbb{R}^{n}$. Descreveremos o cálculo da forma normal usando a expansão da parte não-linear $F$ em série de Taylor até ordem $N$, para isso, precisamos considerar $F$ de classe $C^{N}$.

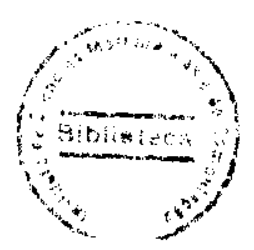


Consirleremos a expansäo de Taylor da parte nào-linear $F$ da forma:

$$
F(u)=\sum_{j \geq 2} \frac{1}{j !} F_{j}(u), u \in C
$$

omk $F_{j}(u)$ é o termo de ordem $j$ na expansáo de Taylor, ou seja. $F_{j}(u)=H_{j}(u, \ldots, u)$ com

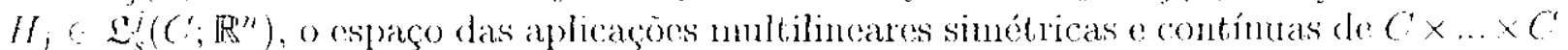
(i)

Definimeto

$$
\begin{aligned}
& f_{j}^{1}(x, y)=\Psi(0) F_{j}(\Phi x+y) \\
& e \\
& f_{j}^{2}(x, y)=(I-\pi) X_{0} F_{j}(\Phi x+y),
\end{aligned}
$$

temos. do sistema (3.10), que:

$$
\left\{\begin{array}{l}
\dot{x}=B x+\sum_{i \geq 2} \frac{1}{i !} f_{i}^{1}(x, y) \\
\frac{d y}{d t}=A_{Q^{1}} y+\sum_{i \geq 2} \frac{1}{i !} f_{i}^{2}(x, y),
\end{array}\right.
$$

com $x \in \mathbb{R}^{m}$ : $y \in Q^{1}=Q \cap C^{1}$.

Calcularemos a forma normal por um processo recursivo, pelo qual determinaremos f'm cata passo os termos de ordem $j \geq 2$ da forma normal a partir dos termos de mesma ordem da equação original e dos termos de ordens inferiores previamente calculados nos passos auteriores. Façamos a mudiança de variáveis:

$$
(x, y) \longmapsto(x, y)+\frac{1}{j !} U_{j}(x)
$$

('om $U_{j}(x)=\left(U_{j}^{1}(x), U_{j}^{2}(x)\right), U_{j}^{1}(x) \in V_{j}^{m}\left(\mathbb{R}^{m}\right), U_{j}^{2} \in V_{j}^{m}\left(Q^{1}\right)$, onde, para um espaço normado $X, V_{j}^{m}(X)$ denota o espaço vetorial dos polinômios homogêneos de grau $j \mathrm{em}$ $m$ variáveis reais $x=\left(x_{1}, \ldots, x_{m}\right)$, com coeficientes no espaço vetorial $X, j \geq 2$, ou seja,

$$
V_{j}^{m}(X)=\left\{\sum_{|q|-j} c_{q} x^{q}: q=\left(q_{1}, q_{2}, \ldots, q_{m}\right) \in \mathbb{N}^{m}, c_{q} \in X\right\},
$$

e nesse espaço consideraremos a norma:

$$
\left|\sum_{|q|=j} c_{q} x^{q}\right| \stackrel{\text { def: }}{=} \sum_{|q|=j}\left|c_{q}\right|_{X}
$$

Faremos a mudança de variável (3.13), com $j=2$, no sistenıa (3.12).

Da primeira equação do sistema (3.12), temos que:

$$
\left(I+\frac{1}{2 !} D_{x} U_{2}^{1}(x)\right) \dot{x}=B\left(x+\frac{1}{2 !} U_{2}^{1}(x)\right)+\sum_{i \geq 2} \frac{1}{i !} f_{i}^{1}\left(x+\frac{1}{2 !} U_{2}^{1}(x), y+\frac{1}{2 !} U_{2}^{2}(x)\right),
$$


- Ha segunda equacio do sistema (3.12):

$$
\frac{d y}{d !}+\frac{1}{2 !} D_{x} U_{2}^{2}(x) x=A_{Q^{\prime}}\left(y+\frac{1}{2 !} U_{2}^{2}(x)\right)+\sum_{i \geq 2} \frac{1}{\pi !} f_{i}^{2}\left(x+\frac{1}{2 !} U_{2}^{1}(x), y+\frac{1}{2 !} U_{2}^{2}(x)\right) \text {. }
$$

'Tomenos o polinomio homogêneo de gran $k$, na variável $(x, y)$ com coeficientes em $\mathbb{A n}^{\prime \prime \prime} \times Q^{1}$ dia forma:

$$
F_{k}(x, y)=\sum_{i m \mid=k} F_{k}^{(m)} x^{p_{1}} x^{p_{2}} \ldots x^{p_{m}} y^{p_{n+1}}
$$

$\operatorname{con} p=\left(p_{1}, p_{2}, \ldots, p_{m}, p_{m+1}\right) \in \mathbb{N}^{m+1}$.

Fazendo a mudança de variável (3.13), obtemos:

$$
F_{k}\left(x+\frac{1}{j !} U_{j}^{1}(x), y+\frac{1}{j !} U_{j}^{2}(x)\right)=F_{k}(x, y)+O\left(|(x, y)|^{(k+2(j-1))}\right) .
$$

Para $x$ suficientemente pequeno, o operador

$$
\left(I+\frac{1}{j !} D_{x} U_{j}^{1}(x)\right)^{-1}
$$

existe e podenos representá-lo em série de potências como segue

$$
\left(I+\frac{1}{j !} D_{x} U_{j}^{1}(x)\right)^{-1}=I-\frac{1}{j !} D_{x} U_{j}^{1}(x)+\frac{1}{(j !)^{2}} D_{x} U_{j}^{1}(x) D_{x} U_{j}^{1}(x)+O\left(|x|^{(3 j-2)}\right) .
$$

Observemos que:

$$
\begin{gathered}
\left(I+\frac{1}{j !} D_{x} U_{j}^{1}(x)\right)\left(I+\frac{1}{j !} D_{x} U_{j}^{1}(x)\right)^{-1}(x)=x \\
\Leftrightarrow-\frac{1}{(j !)^{3}} D_{x} U_{j}^{1}(x) D_{x} U_{j}^{1}(x) D_{x} U_{j}^{1}(x) x+O\left(|x|^{(3 j-2)}\right)=0,
\end{gathered}
$$

on seja, os termos até ordem $2 j-1$ são completamente determinados.

Agora, usando (3.16), conseguimos da equação (3.14).

$$
\left(I+\frac{1}{2 !} D_{x} U_{2}^{1}(x)\right) \dot{x}=B x+\frac{1}{2 !} B U_{2}^{1}(x)+\frac{1}{2 !} f_{2}^{1}(x, y)+\frac{1}{3 !} \tilde{f}_{3}^{1}(x, y)+\cdots,
$$

onde $f_{i}^{1}(x, y) \in V_{i}^{m}\left(\mathbb{R}^{m}\right), i \geq 3$, denota os temos de ordem $i$ na variável $(x, y)$.

Por (3.17), oltemos:

$$
\begin{aligned}
x= & \left(I-\frac{1}{2 !} D_{x} U_{2}^{1}(x)+\frac{1}{(2 !)^{2}} D_{x} U_{2}(x) D_{x} U_{2}(x)+O\left(|x|^{4}\right)\right)\left(B x+\frac{1}{2 !} B U_{2}^{1}(x)+\right. \\
& \left.+\frac{1}{2 !} f_{2}^{1}(x, y)+\frac{1}{3 !} \tilde{f}_{3}^{1}(x, y)+\cdots\right) .
\end{aligned}
$$


Portanto, trmos que:

$$
x=B x+\frac{1}{2 !} y_{2}^{1}(x, y)+\frac{1}{3 !} f_{3}^{1}(x, y)+\cdots,
$$

mule $g_{2}^{1}(x, y)=f_{2}^{1}(x, y)-\left[J_{x} U_{2}^{1}(x) B x-B U_{2}^{1}(x)\right]$ e $f_{i}^{1}(x, y) \in V_{i}^{m}\left(\mathbb{R}^{m}\right), i \geq 3$, renota os termos de ordem i na variável $(x, y)$.

ITsamos (3.16), para obter de (3.15) a seguinte equaçào:

$$
\frac{d y}{d t}=A_{Q^{\prime}} y+\frac{1}{2 !}\left(A_{Q^{\prime}} U_{2}^{2}(x)+\int_{2}^{2}(x, y)-D_{x} U_{2}^{2}(x) \dot{x}\right)+\frac{1}{3 !} j_{3}^{2}(x, y)+\cdots
$$

onde $f_{i}^{2}(x, y) \in V_{i}^{m}(\operatorname{Ker}(\pi)), i \geq 3$, denota os termos de ordem $i$ na variável $(x, y)$.

Substituindo (3.18), obtemos:

$$
\begin{aligned}
\frac{d y}{d !}= & A_{Q^{1}} y+\frac{1}{2 !}\left[A_{Q^{1}} U_{2}^{2}(x)+f_{2}^{2}(x, y)-D_{x} U_{2}^{2}(x)\left(B x+\frac{1}{2 !} g_{2}^{1}(x, y)+\right.\right. \\
& \left.\left.+\frac{1}{3 !} \tilde{f}_{3}^{1}(x, y)+\cdots\right)\right]+\frac{1}{3 !} \tilde{f}_{3}^{2}(x, y)+\cdots
\end{aligned}
$$

ons scijar

$$
\frac{d y}{d t}-A_{Q^{\prime}} y+\frac{1}{2 !} g_{2}^{2}(x, y)+\frac{1}{3 !} \tilde{f}_{3}^{2}(x, y)+\cdots,
$$

onde $g_{2}^{2}(x, y)=f_{2}^{2}(x, y)-\left[D_{r} U_{2}^{2}(x) B x-A_{Q^{1}} U_{2}^{2}(x)\right]$.

Concluímos que mudança de variável (3.13), com $j=2$, no sistema (3.12), nos fornece:

$$
\left\{\begin{aligned}
\dot{x} & =B x+\frac{1}{2 !} g_{2}^{1}(x, y)+\frac{1}{3 !} \tilde{f}_{3}^{1}(x, y)+\cdots \\
\frac{d y}{d t} & =A_{Q^{1}} y+\frac{1}{2 !} g_{2}^{2}(x, y)+\frac{1}{3 !} \tilde{f}_{3}^{2}(x, y)+\cdots
\end{aligned}\right.
$$

coll1

$$
\left\{\begin{array}{l}
g_{2}^{1}(x, y)=f_{2}^{1}(x, y)-\left[D_{x} U_{2}^{1}(x) B x-B U_{2}^{1}(x)\right] \\
g_{2}^{2}(x, y)=f_{2}^{2}(x, y)-\left[D_{x} U_{2}^{2}(x) B x-A_{Q^{1}} U_{2}^{2}(x)\right]
\end{array}\right.
$$

Definiçāo 3.3.1 Para $j \geq 2$, denotamos por $M_{j}$ o operador:

$$
\begin{aligned}
M_{j}(p, h) & =\left(M_{j}^{1} p, M_{j}^{2} h\right), \\
\left(M_{j}^{1} p\right)(x) & =D_{x} p(x) B x-B p(x), \\
\left(M_{j}^{2} h\right)(x) & =D_{x} h(x) B x-A_{Q^{2}} h(x),
\end{aligned}
$$

com donninio $D\left(M_{j}\right)=V_{j}^{n}\left(\mathbb{R}^{m}\right) \times V_{j}^{m}\left(Q^{1}\right)$.

Repetindo o procedimento acima até os tormos de ordem k, obtemos que a forma 
mormal até a ordem té clarla por:

$$
\left\{\begin{array}{l}
\left.x==B x+\sum_{i=2}^{k} \frac{1}{i !} g_{i}^{2}(x, y)+O(\mid(x, y)\}^{k+1}\right), \\
\frac{d y}{d t}=A_{Q^{\prime}} y+\sum_{i=2}^{k} \frac{1}{i !} y_{i}^{2}(x, y)+O\left(|(x, y)|^{k+1}\right) .
\end{array}\right.
$$

onde:

$$
\left\{\begin{array}{l}
g_{i}^{1}(x, y)=\dot{f}_{i}^{1}(x, y)-M_{i}^{1} U_{i}^{1}(x) \\
g_{i}^{2}(x, y)=\tilde{f}_{i}^{2}(x, y)-M_{i}^{2} U_{i}^{2}(x) .
\end{array}\right.
$$

\section{Observações:}

- Como para equações diferenciais ordinárias, ao simplificarmos os temos de ordem $j, j \geq 2$, vemos por $(3.16)$, que os termos de ordem inferior não mudam, mas os termos de ordem superior a $j$ são modificados.

- Os termos que podemos eliminar da equação na forma normal são exatamente os trmos que estäo na imagem do operador $M_{j}$.

Agora. cue sabemos como as mudanças de variáveis agem nas equaçoes, buscaremos as mudanças de variáveis tal que, para cada $j \geq 2$, a função $g_{j}=\left(g_{j}^{1}, g_{j}^{2}\right)$ tesula uma forma simples.

Os termos que surgem na forma normal e nas mudanças de variáveis dependem de como os espaços $V_{j}^{m}\left(\mathbb{R}^{m}\right), V_{j}^{m}(\operatorname{Ker}(\pi))$ e $V_{j}^{m}\left(Q^{1}\right)$ são decompostos en relação à imagem o a) núcleo de $M_{j}^{i}, i=1,2$; que denotaremos por:

$$
\begin{gathered}
V_{j}^{m}\left(\mathbb{R}^{m}\right)=\operatorname{Im}\left(M_{j}^{1}\right) \oplus\left(\operatorname{Im}\left(M_{j}^{1}\right)\right)^{c} \\
V_{j}^{m}\left(\mathbb{R}^{m}\right)=\operatorname{Ker}\left(M_{j}^{1}\right) \oplus\left(\operatorname{Ker}\left(M_{j}^{1}\right)\right)^{c} \\
V_{j}^{m}(\operatorname{Ker}(\pi))=\operatorname{Im}\left(M_{j}^{2}\right) \oplus\left(\operatorname{Im}\left(M_{j}^{2}\right)\right)^{c} \\
V_{j}^{m}\left(Q^{1}\right)=\operatorname{Ker}\left(M_{j}^{2}\right) \oplus\left(\operatorname{Ker}\left(M_{j}^{2}\right)\right)^{c}
\end{gathered}
$$

Tomemos projeções:

$$
\begin{aligned}
& P_{l, j}: \quad V_{j}^{m}\left(\mathbb{R}^{m \prime}\right) \times V_{j}^{m}(\operatorname{Ker} \pi) \rightarrow \operatorname{Im}\left(M_{j}^{1}\right) \times \operatorname{Im}\left(M_{j}^{2}\right) \\
& P_{K, j}: \quad V_{j}^{m}\left(\mathbb{R}^{m}\right) \times V_{j}^{m}\left(Q^{1}\right) \longrightarrow\left(\operatorname{Ker}\left(M_{j}^{1}\right)\right)^{c} \times\left(\operatorname{Ker}\left(M_{j}^{2}\right)\right)^{c},
\end{aligned}
$$

rom $P_{l, j}=\left(P_{l, j}^{1}, P_{I, j}^{2}\right)$ e $P_{K, j}=\left(P_{K, j}^{1} . P_{K, j}^{2}\right)$.

Considerando o operador $M_{j}=\left(M_{j}^{1}, M_{j}^{2}\right)$, definido de

$$
\left[\operatorname{Ker}\left(M_{j}^{1}\right) \oplus\left(\operatorname{Ker}\left(M_{j}^{1}\right)\right)^{c}\right] \times\left[\operatorname{Ker}\left(M_{j}^{2}\right) \oplus\left(\operatorname{Ker}\left(M_{j}^{2}\right)\right)^{c}\right]
$$

sobre

$$
\left[\operatorname{Im}\left(M_{j}^{1}\right) \oplus\left(\operatorname{Im}\left(M_{j}^{1}\right)\right)^{c}\right] \times\left[\operatorname{Im}\left(M_{j}^{2}\right) \oplus\left(\operatorname{Im}\left(M_{j}^{2}\right)\right)^{c}\right]
$$


demotanus por $M_{j}^{-1}=\left(\left(M_{j}^{1}\right)^{-1},\left(M_{j}^{2}\right)^{-1}\right)$ a inversal à direita de $M_{j}$, tal cule:

$$
M_{j}^{-1}\left(\operatorname{Im}\left(M_{j}^{1}\right) \times \operatorname{Im}\left(M_{j}^{2}\right)\right)=\left(\operatorname{Ker}\left(M_{j}^{1}\right)\right)^{r} \times\left(\operatorname{Ker}\left(M_{j}^{2}\right)\right)^{\prime} .
$$

Tomande $y=0$, entrio, $\hat{f}_{j}(x, 0)=\left(\tilde{f}_{j}^{1}(x, 0), \tilde{f}_{j}^{2}(x, 0)\right)$. Nesse caso, a escollha adecinada

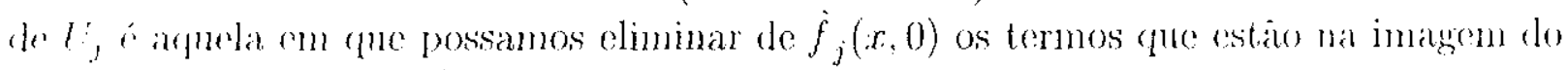
operator $M_{j}=\left(M_{j}^{1} \cdot M_{j}^{2}\right)$, restando somente o termo

$$
g_{j}(x, 0)=\dot{f}_{j}(x, 0) \cdots M_{j} U_{j}(x) .
$$

mats, para isso, precisannos tomar $U_{j}(x) \in\left(V_{j}^{m}\left(\mathbb{R}^{m}\right) \times V_{j}^{m}\left(Q^{1}\right)\right)$ tal que:

$$
M_{j} U_{j}(x)=P_{I, j} \tilde{f}_{j}(x, 0) .
$$

ons sejit,

$$
U_{j}(x)=M_{j}^{-1} P_{I, j} \tilde{f}_{j}(x, 0)
$$

Definição 3.3.2 A forma normal para a equação (3.1), relativa as projeções $P_{1, j}, P_{K, j}$. com! $j \geq 2$, é o sistema em $B C$ :

$$
\left\{\begin{array}{l}
\dot{x}=B x+\sum_{i=2}^{k} \frac{1}{i !} g_{i}^{1}(x, y)+O\left(|(x, y)|^{k+1}\right) \\
\frac{d y}{d t}=A_{Q^{\prime}} y+\sum_{i=2}^{k} \frac{1}{i !} g_{i}^{2}(x, y)+O\left(|(x, y)|^{k+1}\right),
\end{array}\right.
$$

onde $y_{j}=\left(g_{j}^{1}, g_{j}^{2}\right), U_{j}$ e $M_{j}$ sào como cm $(3.19),(3.20)$ e (3.21).

\section{Observaçāo:}

Como os expaços $\left(\operatorname{Im}\left(M_{j}^{i}\right)\right)^{c}$ e $\left(\operatorname{Ker}\left(M_{j}^{i}\right)\right)^{c}, i=1,2$, não são ínicos, a forma normal também não é única e depende da escolha dos espaços $\left(\operatorname{Im}\left(M_{j}^{i}\right)\right)^{c}, i=1,2$, do mesmo modo, a mudança de variável depende da escolla dos espaços $\left(\operatorname{Ker}\left(M_{j}^{i}\right)\right)^{r}$ : $i=1.2$.

\subsection{Condições de Não-Ressonância}

As imagens dos operadores $M_{j}^{1}$ e $M_{j}^{2}, j \geq 2$, definidos, respectivamente, nos espaços $V_{j}^{m}\left(\mathbb{R}^{m}\right)$ e $V_{j}^{m}(\operatorname{ker}(\pi))$, contém exatamente os termos que podem ser climinados da forma normal. Assim, quando essas imagens forem todo o espaço $V_{j}^{m}\left(\mathbb{R}^{m}\right)$ o $V_{j}^{m}(\operatorname{Ker}(\pi))$. respertivanente, podemos eliminar todos os termos de ordem $j$, da primeira e da segunda cfuaçào da forma normal (3.22), respectivamente. Essas situaçóes podem ser caracterizadas en termos do espectro, por apropriadas condiçôes de não ressonància dentre os autovalores da parte linear da equação.

Queremos obter a forma normal sobre uma variedade localmente invariante de dimensào finita, tangente ao subespaço $P$, o qual é invariante pelo gerador infinitesimal $A_{0}$ 
associado a 111 conjunto finito e não-vazio $\Lambda$ de autovalores para o gerador infinitesimal $A_{0}$. Para isso, é necessário que a segunda equação do sistema (3.22), a qual está sobre unn uspaço de dinnemsáo infinitia, seja mula, ou seja, $g_{j}^{2}(x, 0)=0$, para todo $j \geq 2$. Para fliminamos os termos $g_{j}^{2}(x, 0)$, a imagem do operador $M_{j}^{2}$ deve ser necessarianente todo

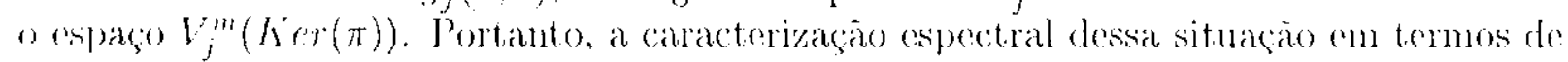
aproprialas condiçós de näo-ressonincia ó essencial para o desenvolvinento da forma mormal.

Os operardores $M_{j}^{1}, j \geq 2$, sĩo os mesmos operadores que surgen no cálculo da forma nomal para equaçós diferenciais ordinárias. Assim, pelo Lema 2.2.1, temos que o espectro de $M_{j}^{1}, j \geq 2$, 6 dado por:

$$
\sigma\left(M_{j}^{1}\right)=\left\{(q, \ddot{\lambda})-\lambda_{j}: i=1, \ldots, m, q=\left(q_{1}, q_{2}, \ldots, q_{m}\right) \in \mathbb{N}^{m}:|q|=j\right\},
$$

onde $\dot{\lambda}=\left\{\lambda_{1}, \ldots, \lambda_{m}\right\},(g, \ddot{\lambda})=q_{1} \lambda_{1}+\ldots+q_{m} \lambda_{m},|q|=q_{1}+\ldots+q_{m}$ e $\lambda_{1}, \ldots, \lambda_{m}$ sào os elementos de $\Lambda$ e cada um deles surgem tantas vezes quanto sua multiplicidade cono raiz da eçuaçióo característica associada.

Esperamos que apropriadas condições de não-ressonància possam garantir que para $y=0$, a forma nomal esteja sobre uma variedade localmente invariante. Nos próximos resultados, estabeleceremos relações entre os espectros de $A, A_{0}$ e $A_{Q^{1}}$.

Lema 3.4.1 O espectro do operador $A$ é igual ao espectro pontual do operador $A$ e ao espectro do operador $A_{0}$, ou seja,

$$
\sigma(A)=\sigma_{p}(A)=\sigma\left(A_{0}\right)
$$

\section{Demonstraçāo:}

Faremos a demonstração desse lena em duas partes, na primeira mostraremos que $\sigma_{p}(A)=\sigma\left(A_{0}\right)$ e, na segunda, mostraremos que $\sigma(A)=\sigma_{p}(A)$.

Provemos que $\sigma_{p}(A)=\sigma\left(A_{0}\right)$.

Sabemos que o espectro de $A_{0}$ é igual ao seu espectro pontual, isto é, $\sigma\left(A_{0}\right)=\sigma_{p}\left(A_{0}\right)$.

- Mostremos que $\sigma\left(A_{0}\right) \subset \sigma_{p}(A)$.

Como $D\left(A_{0}\right)=\left\{\phi \in C: \frac{d \phi}{d \theta} \in C\right.$ e $\left.\frac{d \phi}{d \theta}(0)=\int_{-r}^{\theta}[d \eta(\theta)] \phi(\theta)\right\}$, segue que $D\left(A_{0}\right)$ cim subconjunto de $C^{1}$ e, para $\phi \in D\left(A_{0}\right)$, temos que:

$$
A_{0} \phi=A \phi \text {. }
$$

Se $\lambda \in \sigma_{p}\left(A_{0}\right)$, entĩo existe $\psi \in D\left(A_{0}\right) \backslash\{0\}$, tal que:

$$
\left(A_{0}-\lambda I\right) \phi=0 \text {. }
$$

Daí, conseguimos:

$$
(A-\lambda I) \phi=\left(A_{0}-\lambda I\right) \phi=0 .
$$

Portanto, $\lambda \in \sigma_{p}(A)$.

- Demonstremos (que $\sigma_{p}(A) \subset \sigma\left(A_{0}\right)$. 
Sicja $\lambda \in \sigma_{p}(A)$, entàn existe $\phi \in C^{1} \backslash\{0\}$ satisfazendo:

$$
(A-\lambda I) \phi=0
$$

(1) sisia.

$$
\left\{\begin{array}{c}
\frac{d \phi \phi(\theta)}{d \theta}=\lambda \phi(\theta), \operatorname{se} \theta \in[-r,(\theta) \\
\int_{r}^{0}[d \eta(\theta)] \phi(\theta)=\lambda \phi(\theta), \text { se } \theta=0 .
\end{array}\right.
$$

Conno dis contínua, obtemos:

$$
\frac{d \phi(0)}{d \theta}=\int_{-r}^{0}[d \eta(\theta)] \phi(\theta)
$$

o que implica que $\phi \in D\left(A_{0}\right)$.

Daí, temos que:

$$
\left(A_{0}-\lambda I\right) \phi=(A-\lambda I) \phi=0 .
$$

Iortinto, $\lambda \in \sigma\left(A_{0}\right)$

Agora, mostremos que $\sigma(A)=\sigma_{n}(A)$.

É sufficiente mostrarmos que $\sigma(A) \subset \sigma_{p}(A)$, ou equivalentemente, se $\lambda \notin \sigma_{p}(A)$, entĩo $\lambda \notin \sigma(A)$.

Suponhamos $\lambda \notin \sigma_{p}(A)$, então o operador $(A-\lambda I)$ é inversível. Assim, resta mostrarmos cule a inversa do operador $(A-\lambda I)$ é limitada e tem domínio denso em $B C$. ver $\{1\}$.

Como o operador $A$ é fechado, basta verificarmos que o operador $(A-\lambda I)$ é sobrejetor.

Consideremos $\psi+X_{0} \alpha \in B C$, onde $\psi \in C$ c $\alpha \in \mathbb{R}^{m}$. Mostraremos que existe $\phi \in C^{1}$ tal que:

$$
(A-\lambda I) \phi=\psi+X_{0} \alpha
$$

Pelo Teorema de Riest-Fisher, ver [5], o operador:

$$
\phi \longmapsto L(\phi)-\dot{\phi}(0)
$$

aplica sobrejetivanente $C^{1}$ sobre $\mathbb{R}^{m}$.

Logo, para $\alpha \in \mathbb{R}^{m}$, existe $\phi_{1} \in C^{1}$ tal que:

$$
L\left(\phi_{1}\right)-\dot{\phi}_{1}(0)=\alpha
$$

Agrora, sendo $\sigma\left(A_{0}\right)=\sigma_{p}(A)$, segue que $\lambda \notin \sigma\left(A_{0}\right)$, cultäo para $\psi-\left(\dot{\phi}_{1}-\lambda \phi_{1}\right) \in C$, exist.c $\phi_{2} \in D\left(A_{0}\right)$ satisfazendo:

$$
\left(A_{0}-\lambda I\right) \phi_{2}=\psi \cdots\left(\dot{\phi}_{1}-\lambda \phi_{1}\right) .
$$


Tomemos $\phi=\phi_{1}+\phi_{2} \in C^{1}$, então:

$$
\begin{aligned}
(A-\lambda I) \phi & =(A-\lambda I)\left(\phi_{1}+\phi_{2}\right)=(A-\lambda I) \phi_{1}+\left(A_{0}-\lambda I\right) \phi_{2} \\
& =\left\{\dot{\phi}_{1}+X_{0}\left[L\left(\phi_{1}\right)-\dot{\phi}_{2}(0)\right]-\lambda \phi_{1}\right\}+\left(\psi-\dot{\phi}_{1}+\lambda \phi_{1}\right) \\
& =\psi+X_{0} \alpha .
\end{aligned}
$$

Portanto, o operador $(A-\lambda I)$ é sobrejetor.

Lema 3.4.2 O espectro do operador $A_{Q^{1}}$ coincide com o espectro pontual do operador $A_{Q^{1}}$ e com o espectro do operador $A_{0}$ menos os clementos que estio cm $\Lambda$, ou seja,

$$
\sigma\left(A_{Q^{1}}\right)=\sigma_{p}\left(A_{Q^{1}}\right)=\sigma\left(A_{0}\right) \backslash \Lambda
$$

\section{Demonstração:}

Demonstraremos esse lema em duas etapas; na primcira etapa, mostrarcmos que $\sigma_{p^{\prime}}\left(A_{Q^{1}}\right)=\sigma\left(A_{0}\right) \backslash \Lambda$, e na segunda, provaremos que $\sigma\left(A_{Q^{1}}\right)=\sigma\left(A_{0}\right) \backslash \Lambda$.

Mostremos que $\sigma_{p}\left(A_{\mathcal{Q}^{1}}\right)=\sigma\left(A_{0}\right) \backslash \Lambda$.

- l'rovemos que $\sigma_{p}\left(A_{Q^{1}}\right) \subset \sigma\left(A_{0}\right) \backslash \Lambda$.

Se $\lambda \in \sigma_{p}\left(A_{Q^{1}}\right)$, temos que existe $\phi \in D(A) \backslash\{0\}$ e $\phi \in Q^{1} \backslash\{0\}$ satisfazendo:

$$
\langle A-\lambda I\rangle \phi=0 .
$$

Logo, $\lambda \in \sigma(A)$

Pelo Lema 3.4.1, temos que $\sigma(A)=\sigma\left(A_{0}\right)$ e, portanto, $\lambda \in \sigma\left(A_{0}\right)$.

Suponhamos $\lambda \in \Lambda$, entäo $\phi \in P=\operatorname{ger}\left\{\bigcup_{k=1}^{\infty} \operatorname{Ker}\left((A-\lambda I)^{k}\right)\right.$, com $\left.\lambda \in \Lambda\right\}$, o que $\dot{c}$ un absurdo, una ve que $P \cap Q^{1}=\{0\}$.

Portanto $\lambda \in \sigma\left(A_{0}\right) \backslash \Lambda$.

- Mostremos que $\sigma\left(A_{0}\right) \backslash \Lambda \subset \sigma_{p}\left(A_{Q^{1}}\right)$.

Scja $\lambda \in \sigma\left(A_{0}\right) \backslash \Lambda$, como $\sigma\left(A_{0}\right) \backslash \Lambda=\sigma_{p}\left(A_{0}\right) \backslash \Lambda$, então existe $\phi \in D\left(A_{0}\right) \backslash\{0\}$, tal que:

$$
\left(A_{0}-\lambda I\right) \phi=0 .
$$

Considerando a decomposição de $C$ como em (3.5), decomponos $\phi=\phi^{P}+\phi^{Q}$, onde $\phi^{P} \in P$ e $\phi^{Q} \in Q$. Assim, como o operador $A$ comuta com $\pi$ en $C^{1}$ e $D\left(A_{0}\right) \subset C^{1}$, obtemos:

$$
\left\{\begin{array}{l}
(A-\lambda I) \phi^{P}=\left(A_{0}-\lambda I\right) \phi^{P}=0 \\
(A-\lambda I) \phi^{Q}=\left(A_{0}-\lambda I\right) \phi^{Q}=0 .
\end{array}\right.
$$

Mas, como $\lambda \notin \Lambda$, tenos que $\phi^{P} \equiv 0$.

Logo, $\phi=\phi^{Q} \in Q^{1} \backslash\{0\}$, e obtemos:

$$
(A-\lambda I) \phi=\left(A_{Q^{1}}-\lambda I\right) \phi=0 .
$$


l'orialuta, $\lambda \in \sigma_{p}\left(A_{Q^{1}}\right)$.

Agora. mostromos que $\sigma\left(A_{Q^{\prime}}\right)=\sigma\left(A_{0}\right) \backslash \Lambda$.

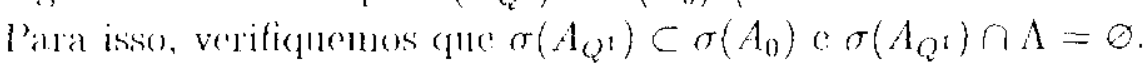

- Misitremos (fler $\sigma\left(A_{Q^{1}}\right) \subseteq \sigma\left(A_{0}\right)$.

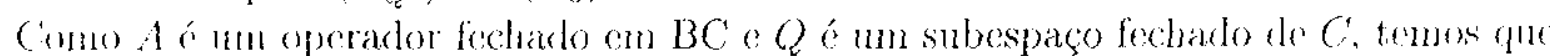
$L_{3}$ i 1110 operador fechado no espaco de Banach Ker $(\pi)$.

Sinpomhamos $\lambda \notin \sigma\left(A_{0}\right)$, sogue do Lema 3.4 .1 que $\sigma\left(A_{0}\right)=\sigma(A)$, entäo, para toda (unciar $f \in \operatorname{Ker}(\pi) \subset B C$, existe $\phi \in C^{1}$, tal que:

$$
(A-\lambda I) \phi=f .
$$

Observemos a d1e $\pi f \equiv 0$, pois $\int \in \operatorname{Ker}(\pi)$.

baí.

$$
(A-\lambda I)(I-\pi) \phi=(I-\pi)(A-\lambda I) \phi=(I-\pi) f=f,
$$

(om $\phi E\left(\operatorname{Her}(\pi) \cap C^{1}\right)=Q^{1}$

Logo, o operador $\left(A_{Q^{1}}-\lambda I\right)$ é sobrejetor e, portanto, $\lambda \notin \sigma\left(A_{Q^{1}}\right)$.

- Provemos que $\sigma\left(A_{Q^{1}}\right) \cap \Lambda=\varnothing$.

Mostrar que $\sigma\left(A_{Q^{3}}\right) \cap \Lambda=\varnothing$ é equivalente a verificar que $\Lambda \subset \rho\left(A_{Q^{1}}\right)$, ou seja, para todo $\lambda \in \Lambda$, o operador $\left(A_{Q^{i}}-\lambda I\right)$ é sobrejetor.

Demonstrenos inicialmente que $\operatorname{Ker}(\pi)=Q \oplus X$, onde:

$$
X=\left\{\phi^{p}+X_{0} \gamma:\left\langle\gamma \in \mathbb{R}^{m} \text { e } \phi^{p}=-\Phi \Psi(0) \propto\right\} .\right.
$$

De fato:

Seja $\phi+X_{0} r \in B C$. Como $\phi \in C=P \oplus Q$, podemos escrever $\phi=\phi^{P}+\phi^{Q}$. com $\phi^{I^{\prime}} \in P^{P} \in \phi^{Q} \in Q$, então $\phi+X_{0} \alpha=\left(\phi^{P}+\phi^{Q}\right)+X_{0} \alpha$.

Daí. segue que $\left(\phi^{P}+\phi^{Q}\right)+X_{0} \alpha \in K e r(\pi)$ se, e somente se,

$$
\pi\left(\left(\phi^{P}+\phi^{Q}\right)+X_{0} \alpha\right)=0
$$

()ll seja

$$
\phi^{P}=-\Phi \Psi(0) \alpha .
$$

Portinto, temos que:

$$
\phi+X_{0} \alpha \in \operatorname{Ker}(\pi),
$$

se $\phi+X_{0} \alpha=\phi^{Q}+\left(\phi^{P}+X_{0} \alpha\right)$, onde $\phi^{P}=-\Phi \Psi(0) \alpha$.

Seja $\psi \in Q \cap X$. Como $\psi \in X$, então

$$
\psi=-\Phi \Psi(0) \alpha+X_{0} \alpha, \alpha \in \mathbb{R}^{n}
$$

Usando o fato de $\psi \in Q$ e portanto deve ser contínua, temos que $\alpha=0$

Logo, $Q \cap X=\{0\}$.

Portanto, $\operatorname{Ker}(\pi)=Q \oplus X$

Agora, mostraremos que $X$ e $Q$ são subconjuntos contidos na imagem do operador 
$\left(t_{Q^{1}}-\lambda I\right)$, para todo $\lambda \in \Lambda$. Conclúndo assim que $\left(A_{Q^{1}}-\lambda I\right)$ é sobrejetor.

- Demonistremos que $Q \subset \operatorname{Im}\left(A_{Q^{2}}-\lambda I\right)$.

Vurifignemos que para carla $f \in Q$, existe $\phi \in Q^{1}$ tal que $\left(A_{Q^{1}}-\lambda I\right) \phi=f$.

P'ara cada $\lambda \in \Lambda$, podenos considerar a decomposiçá:

$$
C=\operatorname{Ker}\left(A_{0}-\lambda I\right)^{k} \oplus \operatorname{Im}\left(A_{0}-\lambda I\right)^{k},
$$

onde ki o o menor inteiro positivo tal cue $\mathcal{M}_{\lambda}\left(A_{0}\right)=\operatorname{Ker}\left(A_{0}-\lambda I\right)^{k}$. Assim, podemos incompor $f \in Q$ da forma:

$$
f=\phi_{1}+\left(A_{0}-\lambda I\right)^{k} \phi_{2}
$$

com $\phi_{1}, \phi_{2} \in D\left(\left(A_{0}-\lambda I\right)^{k}\right) \cong\left(A_{0}-\lambda I\right) \phi_{1} \equiv 0$. Fm particular, $\phi_{1} \in P$.

Como $f \in Q$, temos que:

$f=(I-\pi) f=(I-\pi)\left(\phi_{1}+\left(A_{0}-\lambda I\right)^{k} \phi_{2}\right)=\left(A_{0}-\lambda I\right)(I-\pi)\left(A_{0}-\lambda I\right)^{k-1} \phi_{2}=\left(A_{0}-\lambda I\right) \phi$, onde $\phi=(I-\pi)\left(A_{0}-\lambda I\right)^{k-1} \phi_{2}$, e mais, $\phi \in\left(D\left(A_{0}\right) \cap \operatorname{Ker}(\pi)\right) \subset(D(A) \cap \operatorname{Ker}(\pi))=Q^{1}$.

Logo, $\left(A_{Q^{1}}-\lambda I\right) \phi=\int$.

Portatito, $Q \subset \operatorname{Im}\left(A_{Q^{1}}-\lambda I\right)$.

- Mostremos que $X \subset \operatorname{Im}\left(A_{Q^{1}}-\lambda I\right)$.

Observemos que, para $f \in X$, podemos escrever $f=\phi^{F}+X_{0} \alpha$, com $\alpha \in \mathbb{R}^{n}$ e $\phi^{r}=-\Phi \Psi(0) \alpha$, então:

$$
\begin{aligned}
(I-\pi) f & =(I-\pi)\left(\phi^{P}+X_{0} \alpha\right)=\left(\phi^{P}+X_{0} \alpha\right)-\pi\left(\phi^{P}+X_{0} \alpha\right) \\
& =\left(\phi^{P}+X_{0} \alpha\right)-\left(\phi^{P}+\Phi \Psi(0) \alpha\right)=\left(\phi^{P}+X_{0} \alpha\right)=f .
\end{aligned}
$$

Assim, provarmos que existe $h \in Q^{1}$, tal que:

$$
\left(A_{Q^{1}}-\lambda I\right) h=f \text {, }
$$

6́ cquivalente à mostrarmos que:

$$
(A-\lambda I)(I-\pi) h=(I-\pi) f \Leftrightarrow(A-\lambda I) h=f,
$$

que pela definição do operador $A$, equivale d̀:

$$
\left\{\begin{array}{l}
\dot{h}_{h}(\theta)-\lambda h(\theta)=-\Phi(\theta) \Psi(0) \alpha, \theta \in[-r, 0) . \\
L(h)-\dot{h}_{h}(0)=\alpha .
\end{array}\right.
$$

A solução da primeira equação do sistema (3.23) é da forma:

$$
h(\theta)=e^{\lambda \theta} h(0)-e^{\lambda \theta} \int_{0}^{\theta} e^{-\lambda t} \Phi(t) \Psi(0) \alpha d t .
$$


Mas, $h \subset C^{1}$ satisfaz o sistema (3.23), se:

$$
\int_{-r}^{0}[d \eta(\theta)] h(\theta)-(\lambda h(0)-\Phi(0) \Psi(0) \alpha)=\alpha .
$$

Substituindo a expressão para $h(\theta)$, obtemos que:

$$
\begin{aligned}
\Delta(\lambda) h(0) & =\left[-I+\Phi(0) \Psi(0)-L\left(e^{\lambda \theta} \int_{0}^{\theta} e^{-\lambda t} \Phi(t) \Psi(0) d t\right)\right] \alpha \\
& =\left\{-I+\left[\Phi(0)-\int_{-r}^{0}[d \eta(\theta)] \int_{0}^{\theta} e^{\lambda(\theta-t)} \Phi(t) d t\right] \Psi(0)\right\} \alpha \\
& =\left[-I+\left(e^{-\lambda \theta} I, \Phi\right) \Psi(0)\right] \alpha,
\end{aligned}
$$

onde $\Delta(\lambda)=\lambda I-L\left(e^{\lambda \theta} I\right)$ e $(\cdot, \cdot)$ é a forma bilinear.

Portanto, a existência do $h \in C^{1}$ satisfazendo o sistema (3.23), é equivalente a existencia de $h(0) \in \mathbb{R}^{n}$ tal que:

$$
\Delta(\lambda) h(0)=\left[-I+\left(e^{-\lambda \theta} I, \Phi\right) \Psi(0)\right] \alpha .
$$

Assim, resta apenas mostrarmos que as colunas da matriz $n \times n$,

$$
\mathfrak{A}(\lambda) \stackrel{d e f .}{=}-I+\left(e^{-\lambda} I, \Phi\right) \Psi(0),
$$

sào geradas pelas colunas da matriz $\Delta(\lambda)$.

Seja $\lambda \in \Lambda$. Escolhemos uma base $\left\{c_{1}, \ldots, c_{n}\right\}$ para $\mathbb{C}^{n}$, na qual, $\Delta(\lambda)$ está na forma canônica de Jordan, com os primeiros blocos associados com os autovalores nulos de $\Delta(\lambda)$. Suponhamos que existam $R$ blocos de Jordan associados com os autovalores nulos e (1ue o número de colunas (ou linhas) desses blocos sejam $n_{1}, \ldots, n_{R}$. A primeira coluna e a última linha em cada bloco de Jordan de $\Delta(\lambda)$ associado com o autovalor nulo, são nulas e as colunas restantes são independentes. Portanto, provar que o espaço coluna de $\mathfrak{A}(\lambda)$ está contido no espaço coluna de $\Delta(\lambda)$, é equivalente a mostrar que as linhas $n_{1}, n_{1}+n_{2}, \ldots, n_{1}+n_{2}+\ldots+n_{R}$ de $\mathfrak{A}(\lambda)$ săo nulas. Sem perda de generalidade, provaremos que a linha $n_{1}$ de $\mathfrak{A}(\lambda)$ é nula, uma vez que, o anulamento das outras linhas inencionadas, segue desse caso por meio de uma reorganização da base.

Considermos uma base $\Psi=\operatorname{col}\left(\psi_{1}, \ldots, \psi_{m}\right)$ de $P^{T}$ tal que a matriz $B$, satisfazendo:

$$
-\dot{\Psi}=B \Psi
$$

esteja na forma canônica de Jordan, com os primeiros blocos associados ao autovalor $\lambda$, e denotamos por $m_{1}$ o número de colunas (linlass) do primeiro bloco de Jordan. A linha $n_{1}$ de $2(\lambda)$ e:

$$
\mathfrak{A}_{n_{1}}(\lambda)=-e_{n_{1}}^{T}+\left(e^{\cdots \lambda} e_{n_{1}}^{T}, \Phi\right) \Psi(0),
$$

onde $e_{i}^{T}=\left(\delta_{1, i}, \ldots, \delta_{n, i}\right)$, com $\delta_{j, i}=1$ se $j=i$ e $\delta_{j, i}=0$ se $j \neq i$.

Como $B$ está na forma canônica de Jordan, com o primeiro bloco associado com o antovalor $\lambda$, temos que:

$$
-\dot{\psi}_{m_{1}}=\lambda \psi_{m_{1}} \text {. }
$$




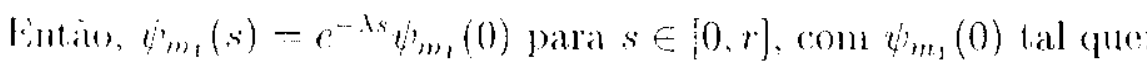

$$
\psi^{\prime}, m_{1} \in D\left(A_{0}^{\prime}\right)=\left\{\psi \in\left(C^{T}\right)^{1}:-\dot{\psi}(0)=\int_{-r}^{0} \psi(-\theta)[d \eta(\theta)]\right\},
$$

onde $A_{0}^{T}$ s o gerador infinitesimal do semigrupo definido pela equaçào adjunta.

Daí, obtemos que:

$$
\begin{aligned}
\psi_{m_{1}}(0) \Delta(\lambda) & =\psi_{m_{1}}(0)\left(\lambda I-L\left(e^{\lambda \theta} I\right)\right)=\lambda \psi_{m_{s}}(0)-\int_{-r}^{0} \psi_{m_{1}}(0)[d \eta(\theta)] e^{\lambda \theta} \\
& =-\psi_{m_{2}}(0)-\int_{-r}^{0} e^{\lambda \theta} \psi_{m_{1}}(0)[d \eta(\theta)] \\
& =\int_{-r}^{0} \psi_{m_{1}}(-\theta)[d \eta(\theta)]-\int_{-r}^{0} e^{\lambda \theta} \psi_{m_{1}}(0)[d \eta(\theta)] \\
& =\int_{-r}^{0} \psi_{m_{1}}(-\theta)[d \eta(\theta)]-\int_{-r}^{0} \psi_{m_{1}}(-\theta)[d \eta(\theta)]=0 .
\end{aligned}
$$

Desse modo, como a linha $n_{1}$ de $\Delta(\lambda)$ é mula, temos que:

$$
e_{n_{1}}^{T} \Delta(\lambda)=0
$$

logo. podemos tomar $\psi_{m_{1}}(0)=e_{n_{1}}^{T}$.

Entào,

$$
\psi_{m_{1}}(s)=e^{-\lambda s} e_{n_{1}}^{T},
$$

para $s \in[0, r]$.

Considerando $(\Psi, \Phi)=I$, cada elemento $\phi_{i}$ da base $\Phi$ satisfaz:

$$
\left(e^{-\lambda} e_{n_{1}}^{T}, \phi_{i}\right)=\left(\psi_{m_{1}}, \phi_{i}\right)= \begin{cases}0 & \text { se } i \neq m_{1} \\ 1 & \text { se } i=m_{1}\end{cases}
$$

Portanto, obtemos:

$\mathfrak{A}_{n_{1}}(\lambda)=-e_{n_{1}}^{T}+\left(e^{-\lambda} e_{n_{1}}^{T}, \Phi\right) \Psi(0)=-e_{n_{1}}^{T}+\left(\psi_{m_{1}}, \Phi\right) \Psi(0)=-e_{n_{1}}^{T}+\psi_{m_{1}}(0)=0$.

Conchúmos assim, que a linha $n_{1}$ de $\mathfrak{A}(\lambda)$ é nula.

O próximo teorema nos dará os espectros dos operadores $M_{j}^{2}, j \geq 2$.

Teorema 3.4.1 Os espectros dos operadores $M_{j}^{2}$, para $j \geq 2$, definidos em (3.19), sào dados por:

$$
\sigma\left(M_{j}^{2}\right)=\sigma_{p}\left(M_{j}^{2}\right)=\left\{(q, \bar{\lambda})-\mu: \mu \in \sigma\left(\Lambda_{0}\right) \backslash \Lambda, q=\left(q_{1}, \ldots, q_{m}\right) \in \mathbb{N}^{m},|q|=j\right\} .
$$

com. $\lambda=\left(\lambda_{1}, \ldots, \lambda_{m}\right),(q, \bar{\lambda})=q_{1} \lambda_{1}+\cdots+q_{m} \lambda_{m} e|q|=q_{1}+\cdots+q_{m}$. 


\section{Demonstraçāo:}

Consideremos wm sistema de coordenadas tal (que a matriz $B$ esteja na forma canonica de forrlan:

$$
B=\left[\begin{array}{ccccc}
\lambda_{1} & \sigma_{1} & 0 & \cdots & 0 \\
0 & \lambda_{2} & \sigma_{2} & \cdots & 0 \\
\vdots & \vdots & \ddots & \vdots & \sigma_{m-1} \\
0 & 0 & \cdots & 0 & \lambda_{m}
\end{array}\right]
$$

(o) $\sigma_{i}=1$ on $\sigma_{i}=0$.

Tomemos $h \in V_{j}^{m}\left(Q^{1}\right), f \in V_{j}^{m}(\operatorname{Ker}(\pi))$,

$$
\begin{aligned}
& h(x)=\sum_{|q|=j} h_{q} x^{q}, \operatorname{com} h_{q} \in Q^{1}, \\
& f(x)=\sum_{|q|=j} f_{q} x^{q}, \operatorname{com} f_{q} \in \operatorname{Ker}(\pi) .
\end{aligned}
$$

Definimos $D_{j}$ o conjunto dos índices $q=\left(q_{1}, \ldots, g_{m}\right) \in \mathbb{N}^{m}$ de grau $j$, ou seja,

$$
D_{j}=\left\{q=\left(q_{1}, \ldots, q_{m}\right) \in \mathbb{N}^{m} \text { tal que }|q|=j\right\} .
$$

Sejam $p=\left(p_{1}, \ldots, p_{m}\right), q=\left(q_{1}, \ldots, q_{m}\right) \in D_{j}$, dizennos que $p<q$ se a primeira diferença não mula de $p_{1}-q_{1}, \ldots, p_{m}-q_{m}$ for positiva.

De nesmo modo que fizenos no Lema 2.2.1, mostramos que:

$$
\sigma_{p}\left(M_{j}^{2}\right)=\left\{(q, \bar{\lambda})-\mu \text { tal que } \mu \in \sigma\left(A_{0}\right) \backslash \Lambda, q=\left\{q_{1}, \ldots, q_{m}\right\} \in \mathbb{N}^{m} \in|q|=j\right\} .
$$

Assim, resta mostrarmos que $\sigma\left(M_{j}^{2}\right) \subset \sigma_{p}\left(M_{j}^{2}\right)$, ou seja, todo $\alpha \notin \sigma_{p}\left(M_{j}^{2}\right)$ pertence à $\rho\left(M_{j}^{2}\right)$.

Obscrvemos que os operadores $M_{j}^{2}, j \geq 2$, são fechados em $V_{j}^{m}(\operatorname{Ker}(\pi))$ e, portanto, basta verificarmos que os operadores $\left(\alpha I-M_{j}^{2}\right), j \geq 2$, são sobrejetores.

Para cada $q \in D_{j}$, se $\mu_{q}=(q, \vec{\lambda})-\alpha, \phi \in Q^{1}$ e $\psi \in \operatorname{Ker}(\pi)$, são tais que,

$$
\begin{aligned}
& \psi=\left(A_{Q^{1}}-\mu_{q} I\right) \phi, \\
& h(x)=\phi x^{q} \in V_{j}^{m}\left(Q^{1}\right), \\
& f(x)=\left(\alpha x-M_{j}^{2}\right) h(x) .
\end{aligned}
$$

Entè̃o:

$$
\begin{aligned}
f(x) & =\left(\alpha I-M_{j}^{2}\right) h(x)=\alpha h(x)-\left[D_{x} h(x) B x-A_{Q^{1}} h(x)\right] \\
& =\alpha\left(\phi x^{g}\right)-\left[D_{x}\left(\phi x^{g}\right) B x-A_{Q^{1}}\left(\phi x^{q}\right)\right]
\end{aligned}
$$


$=\left(A_{Q^{1}}+\alpha\right)\left(\phi x^{q}\right)-D_{r}\left(\phi x^{q}\right) B x$

$=\left(A_{Q^{\prime}}+(x)\left(\phi x^{q}\right)-\right.$

$$
\begin{aligned}
& \cdots\left[\begin{array}{llll}
q_{1} \phi x^{4} \cdots & \eta_{2} \phi x^{q-\rho_{2}} & \ldots & q_{m} \phi x^{q-m_{m}}
\end{array}\right]\left[\begin{array}{cccc}
\lambda_{1} & \sigma_{1} & \ldots & 0 \\
0 & \lambda_{2} & \ldots & 0 \\
\vdots & \vdots & \ddots & \vdots \\
0 & 0 & \ldots & \lambda_{m}
\end{array}\right]\left[\begin{array}{c}
r_{1} \\
r_{2} \\
\vdots \\
r_{m}
\end{array}\right] \\
& =\left(A_{Q^{1}}+\alpha\right)\left(\phi x^{q}\right)-\sum_{i=1}^{m} q_{i} \phi x^{q-c_{i}}\left(\lambda_{i} x_{i}+\sigma_{i} x_{i+1}\right) \\
& =\left(A_{Q^{1}}+\alpha\right)\left(\phi x^{4}\right)-\sum_{i=1}^{m} q_{i} \lambda_{i} \phi x^{q}-\sum_{i=1}^{m-1} q_{i} \sigma_{i} \phi x^{q+\epsilon_{i+1}-\epsilon_{i}} \\
& =\left(A_{Q^{1}}-((q, \lambda)-\alpha)\right)\left(\phi x^{q}\right)-\sum_{i=1}^{m-1} q_{i} \sigma_{i} \phi x^{q+r_{i+1}-r_{i}} \\
& =\left(A_{Q^{1}}-\mu_{q}\right)\left(\phi x^{g}\right)-\sum_{i=1}^{m-1} q_{i} \sigma_{i} \phi x^{g+e_{i+1}-e_{i}} \\
& =\psi x^{q}-\sum_{i=1}^{m-1} q_{i} \sigma_{i} \phi x^{q+c_{i-1}-e_{i}},
\end{aligned}
$$

onde $e_{i}=\left(\delta_{1, i}, \ldots, \hat{\delta}_{m, i}\right), \delta_{i, j}=1$ se $i=j$ e $\delta_{i, j}=0$ se $i \neq j$.

Como podemos escrever $f(x)$ na forma:

$$
f(x)=\sum_{|p|=-j} f_{p} x^{p}
$$

obtemos que

$$
f_{p}=\left\{\begin{array}{cl}
\psi, & \text { se } p=q, \\
-q_{i} \sigma_{i} \phi, & \text { se } p=q+e_{i-1}-e_{i} \text { para algum } i=1,2, \ldots, m-1 . \\
0, & \text { caso contrário. }
\end{array}\right.
$$

Seja $g \in V_{j}^{m}(\operatorname{Ker}(\pi))$, dada por:

$$
g(x)=\sum_{|p|=j} g_{p} x^{p},
$$

mostraremos que existe $\tilde{h} \in V_{j}^{m}\left(Q^{1}\right)$ satisfazendo:

$$
\left(\alpha I-M_{j}^{2}\right) \tilde{h}(x)=g(x) .
$$


Provaremos por induçäo sobre os elementos de $D_{j}$, que para cada $q \in D_{j}$, existe $h^{\prime \prime} \in \lim ^{m}\left(Q^{1}\right)$ tal que:

$$
\left[\left(\alpha I-M_{j}^{2}\right) h^{q}\right]_{p}=g_{p}, \text { para } p \leq q .
$$

Parai o prineiro elemento de $D_{j}, q=(j, 0, \ldots, 0)$, temos que:

$$
\mu_{q}=(q, \bar{\lambda})-\alpha \in \rho\left(\Lambda_{Q^{\prime}}\right) \text {, }
$$

pris, $n \not \sigma_{p}\left(M_{j}^{2}\right)$ e pelo Loma 3.4.2, $\sigma\left(A_{Q^{3}}\right)=\sigma\left(A_{0}\right) \backslash \Lambda$

P'ortanto, para cada $g_{q} \in \operatorname{Lier}(\pi)$, existe $\phi_{q} \in Q^{1}$ tal que:

$$
\left(A_{Q^{1}}-\mu_{q} I\right) \phi_{q}=g_{q}
$$

Definindo $h^{q}(x)=\phi_{q} x^{q}$, obtemos de (3.24) que:

$$
\left[\left(\alpha I-M_{j}^{2}\right) h^{q}\right]_{q}=g_{q} .
$$

Hipótese de Indução: Suponhanos que para um certo $q \in D_{j}$, exista $h^{q} \in V_{j}^{m}\left(Q^{1}\right)$ satisfazendo

$$
\left[\left(\alpha I-M_{j}^{2}\right) h^{q}\right]_{p}=g_{p}, \text { para } p \leq q .
$$

Seja $\bar{q} \in D_{j}$ o sucessor de $q$ na ordenação considerada, definimos

$$
H_{\tilde{q}}=\left[\left(\alpha I-M_{j}^{2}\right) h^{q}\right]_{\tilde{q}} \in \operatorname{Ker}(\pi) .
$$

Como $\mu_{q}=(q: \ddot{\lambda})-r \in \rho\left(A_{Q^{1}}\right): g_{\bar{q}}-H_{\tilde{q}} \in \operatorname{Ker}(\pi)$, existe $\phi_{\bar{q}} \in Q^{1}$ tal que:

$$
\left(A_{Q^{1}}-\mu_{\tilde{q}} I\right) \phi_{\tilde{q}}=g_{\tilde{q}}-H_{\bar{q}}
$$

Assim, considerando o polinomio homogèneo $H^{\dot{q}}=\phi_{\dot{q}} x^{\dot{q}} \in V_{j}^{m}\left(Q^{\prime}\right)$ e usando (3.24), obtemos:

$$
\left[\left(\alpha I-M_{j}^{2}\right) H^{\grave{q}}\right]_{p}=\left\{\begin{array}{cl}
g_{\bar{q}}-H_{\bar{q}}, & \text { se } p=\tilde{q}, \\
-\tilde{q}_{i} \sigma_{i} \phi_{\bar{q}}, & \text { se } p=\tilde{q}+e_{i-1}+e_{i} \text { para algum } i=1, \ldots, m-1, \\
0, & \text { caso contrário. }
\end{array}\right.
$$

Tomamos $h^{\tilde{q}}=h^{q}+H^{\tilde{q}} \in V_{j}^{m}\left(Q^{1}\right)$, como $q<\tilde{q}<\tilde{q}+e_{i-1}+e_{i}$ c usando a lipótese de induçăo, conseguimos:

$$
\begin{aligned}
& {\left[\left(\alpha I-M_{j}^{2}\right) h^{\tilde{q}}\right]_{p}=\left[\left(\alpha I-M_{j}^{2}\right) h^{q}\right]_{p}+\left[\left(\alpha I-M_{j}^{2}\right) H^{\dot{q}}\right]_{p^{\prime}}} \\
& = \begin{cases}g_{p}, & \text { se } p \leq q, \\
{\left[\left(\alpha I-M_{j}^{2}\right) h^{q}\right]_{\tilde{q}}+g_{\tilde{q}}-H_{\tilde{q}},} & \text { se } p=\tilde{q},\end{cases} \\
& = \begin{cases}g_{p}, & \text { se } p \leq q, \\
g_{\tilde{q}}, & \text { se } p=\bar{q} .\end{cases}
\end{aligned}
$$


Portanto, temos que:

$$
\left[\left(\alpha I-M_{j}^{2}\right) h^{i j}\right]_{p}=g_{p}, \text { para } p \leq \tilde{q}
$$

Finalizanues assim a prova por indução.

Tomemos $q \in D_{j}$ conno sendo o maior elemento de $D_{j}$, ention, existe $h^{\prime} \in V_{j}^{m}\left(Q^{1}\right)$ satisfarendo:

$$
\left[\left(\alpha J-M_{j}^{2}\right) h^{y}\right]_{p}=g_{p}, \text { para todo } p \in D_{j} \text { e } j \geq 2
$$

Por últino, defininde $h(x)=\sum_{|p|=j} h^{q} x^{p}$, obtemos:

$$
\begin{aligned}
\left(\gamma I-M_{j}^{2}\right) \tilde{h}(x) & =\sum_{: p \mid=j}\left(\alpha I-M_{j}^{2}\right)\left(h^{q}: x^{p}\right)=\sum_{|p|=j}\left[\left(\alpha I-M_{j}^{2}\right) h^{q}\right]_{p}, x^{p} \\
& =\sum_{|p|=j} g_{p} \cdot x^{p}=g(x) .
\end{aligned}
$$

Portanto, o operalor $\left(\alpha I-M_{j}^{2}\right)$ é sobrejetor.

Agora, podemos dar a caracterização espectral da situação descrita no início dessa seçào.

Definição 3.4.1 Dizemos que a equaçio (3.1) safisfaz as condiçoes de năo-ressonancia rlatimumente is $\Lambda \subset \sigma\left(A_{0}\right)$, se

$$
(q, \bar{\lambda}) \neq \mu \text { para todo } \mu \in \sigma\left(A_{0}\right) \backslash \Lambda, q=\left(q_{1}, \ldots, q_{m}\right) \in \mathbb{N}^{m} \text { e }|q| \geq 2,
$$

onde $\bar{\lambda}=\left(\lambda_{1}, \ldots, \lambda_{m}\right)$ e $\lambda_{1}, \ldots, \lambda_{m}$ são os elementos de $\Lambda$, e cada um deles aparecem tantas vezes quanto sua multiplicidade como raiz da equaçâo característica dada por (3.3).

Corolário 3.4.1 Suponhamos que as condições de näo-ressonância da Definiçäo 3.4.1 sàn satisfeitas. Então,

$$
\operatorname{Im}\left(M_{j}^{2}\right)=V_{j}^{m}(\operatorname{Ker}(\pi)),
$$

para todo $j \geq 0$, on seja, podemos eliminar todos os termos $g_{j}^{2}$ da forma normal.

\section{Demonstração:}

Como podemos escrever o espaço $V_{j}^{m}(\operatorname{Ker}(\pi))$ como soma direta da imagem e do núcleo do operador $M_{j}^{2}$, é suficiente verificarmos que o núcleo do operador $M_{j}^{2}$ contém apenas o polinomio identicanente nulo.

Suponhamos que existe $g(x) \in \operatorname{Ker}\left(M_{j}^{2}\right) \backslash\{0\}$, entiro temos que:

$$
M_{j}^{2} g(x) \equiv 0,
$$

() que implica que 0 pertence ao espectro de $M_{j}^{2}$, contradizendo as condições de nãoressonanciacia. 
Trorema 3.4.2 Consideremos a equaçüo (3.1) e a decomposiçán de C, deda por:

$$
C=P \oplus Q
$$

onde P' o subespaço imariante pelo gerador infinitesimal $A_{0}$. associado ao subconImuto nio vasio c finito $\Lambda$ de autovalores. Assim, temos a decomposigño $u=\Phi x+y$. ande $x \in \mathbb{R}^{m}, y \in Q, \operatorname{dim} P=m$ e $\Phi$ é uma base para $P$. Se as combicoes de nooresonameria relativamente à $\Lambda$ da Definiçäo 3.4 .1 sầ salisfeitas, entào. existe uma mu-

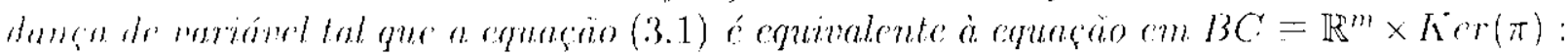

$$
\left\{\begin{array}{l}
\dot{x}=B x+\sum_{j \geq 2} \frac{1}{j !} g_{j}^{1}(x, y), \\
\dot{y}=A_{Q^{1}} y+\sum_{j \geq 2} \frac{1}{j !} g_{j}^{2}(x, y),
\end{array}\right.
$$

onde $g_{j}^{1}, g_{j}^{2}$ são obtidos como em $(3.20)$, com $g_{j}^{2}(x, 0)=0$, para $j \geq 2$. Essa equação está na forma normal relativamente à $P$. Se existir uma varicdade localmente invariante para a cquação (3.1), tangente à $P$ no zero, então, essa satisfaz $y=0$ e o fuxo sobre essa variclade é dado pela equagcio diferencial ordinária m-dimensional:

$$
\dot{x}=B x+\sum_{j \geq 2} \frac{1}{j !} g_{j}^{1}(x, y)
$$

a qual está na forma normal, no sentido usual para equaçòes diferenciais ordinárias.

\section{Observação:}

Em muitos casos em que as condições de não-ressonância são satisfeitas, há um particular interesse pelas aplicações onde a decomposição de $C$ está associada ao conjunto (i) $\Lambda=\{\lambda \in \sigma: \operatorname{Re}(\lambda)=0\}$ ou (ii) $\Lambda=\{\lambda \in \sigma: \operatorname{Re}(\lambda) \geq 0\}$. No caso (i), para $y=0$, a forma normal (3.26) está sobre a variedade central, e no caso (ii), sobre a variedade contral-inst,ível.

\subsection{Equações Diferenciais Funcionais com Parâmetro}

Nas duas seções seguintes, obteremos a forma normal para equaçòes diferenciais funcionais retardadas com um parâmetro em um espaço invariante de dimensão finita associado a um conjunto finito de autovalores do gerador infinitesimal da equação linearizada na singularidade.

Em muitos casos, é conveniente introdızirmos um novo parâmetro na equação difercucial funcional retardada, para que possamos descrever completamente a singularidade. Desenvolveremos a forma normal para equaçós diferenciais funcionais retardadas com um parâmetro acrescentando uma nova equação, o que possibilitará considerarmos o parâmetro como uma nova variável.

Consideremos uma equação diferencial funcional retardada com um parâmetro,

$$
\dot{z}(t)=L(\alpha)\left(z_{t}\right)+F\left(z_{t}, \alpha\right),
$$


onde $z_{t}$ pertence ì $C=C\left([-r, 0], \mathbb{R}^{n}\right)$ o o parametro $\alpha$ pertence a uma vizinlança $V$ do

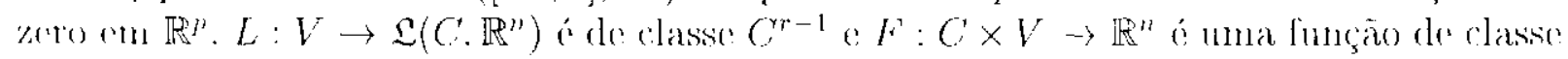
$(" c) m P(0.0)=0$ c $D F(0,0)-0$

Considerarmos o parâmetro a como unia nova variável. Para isso, estudenuos o sislemia:

$$
\left\{\begin{aligned}
\dot{\tilde{z}}(t) & =L(\alpha)\left(z_{t}\right)+F\left(z_{t}, \alpha\right) \\
\dot{\alpha} & =0 .
\end{aligned}\right.
$$

Para desenvolvermos a forma normal para o sistema do equaçoes diferenciais funcionais retardadas (3.28), basta eonsiderarmos a primeira equaçào do sistema, já (pue, a segunda equação está na forma mais simples possível.

Definindo $L_{0}=L(0)$, segue que,

$$
L(\alpha)\left(z_{t}\right)-L_{0}\left(z_{t}\right)=O\left(\mid\left(z_{t} \cdot \alpha\right)_{\mid}^{2}\right) .
$$

e portanto, podemos resserever a equação (3.27),

$$
\dot{z}(t)=L_{0}\left(z_{t}\right)+\left[F\left(z_{t}, \alpha\right)+L(\alpha)\left(z_{t}\right)-L_{0}\left(z_{t}\right)\right],
$$

onde $\left[F\left(\tilde{z}_{t}, \alpha\right)+L(\alpha)\left(z_{t}\right)-L_{0}\left(z_{t}\right)\right]$ é a parte nào linear. nas variáveis z e $\alpha$.

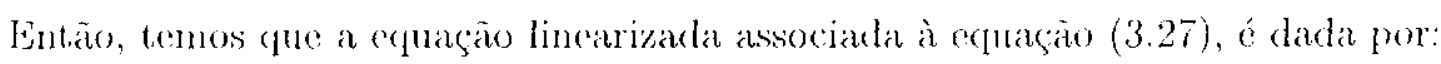

$$
\dot{z}(t)=L_{0}\left(z_{t}\right) \text {. }
$$

Sejam $T(t), t \geq 0$, o $C_{0}$-semigrupo associado à equação linearizada e $A_{0}$ o gerador infinitesimal associado ao $C_{0}$-semigrupo $T(t), t \geq 0$. Tomemos $\Lambda$ um subconjunto finito e não-vazio de autovalores do gerador infinitesimal $A_{0}$, e supomos que $\Lambda$ contém $m$ elencutos onde cada elemento é contado tantas vezes quanto sua multiplicidade.

Como vimos na Seção 3.2 , para obtermos a equação diferencial ordinária abstrata associada à equação (3.29), com a decomposição dos termos lineares e não lineares da equação bem formulada, precisamos considerar o espaço de fase $B C$, sobre o qual a equação diferencial ordinária abstrata, ó darla por:

$$
\frac{d v}{d t}=A v+X_{0} G(v, \alpha)
$$

onde

$$
\begin{gathered}
A \phi=\dot{\phi}+X_{0}\left[L_{0}(\phi)-\dot{\phi}(0)\right], \\
G(\phi, \alpha)=F(\phi, \alpha)+\left[L(\alpha)(\phi)-L_{0}(\phi)\right]
\end{gathered}
$$

e com domínio $D(A)=C^{1}$.

Dá, da mesna forma que fizemos na Seção 3.2 , obtemos que a equação diferencial ordinária abstrata (3.30), pode ser decomposta por:

$$
\left\{\begin{aligned}
\dot{x} & =B x+\Psi(0) G(\Phi x+y, \alpha), \\
\frac{d y}{d t} & =A_{Q^{1}} y+(I-\pi) X_{0} G(\Phi x+y, \alpha) .
\end{aligned}\right.
$$


oude $t_{Q}$ é a restriça do operador $A$ ao subespaço $Q^{1}$ de $B Q, x \in \mathbb{R}^{m}$ a $y \in Q^{2}$

\subsection{Formas Normais}

Nesta seçà calcularemos a forma normal para equações diferenciais funcionais retalrdadas eom mon parânetro, para isso. faremos uso do algoritmo previamente desenvolvido Ia Secito 3.3

Comsirlerenos as expansón de Taylor:

$$
\begin{aligned}
L(\alpha) & =L_{0}+\sum_{j=2}^{r-1} L_{j}(\alpha)+O\left(|\alpha|^{r-1}\right), \\
F\left(z_{t}, \alpha\right) & =\sum_{j=2}^{r-1} \frac{1}{j !} F_{j}\left(z_{t}, \alpha\right)+O\left(\left|\left(z_{t}, \alpha\right\rangle\right|^{r}\right),
\end{aligned}
$$

onte $L_{j}(\alpha)$ é de orden j -1 en $\alpha$ e $F_{j}\left(z_{t}, \alpha\right)$ é de ordem jem $\left(z_{t}, \alpha\right)$. Notemos que $L_{j}(\alpha) z_{f}$ é de ordem jem $\left(z_{t}, \alpha\right)$.

lela dreomposiçán de $C$, como en $(3.5)$, pordenos escrever $z_{t}=\Phi x+y$, com $x \in \mathbb{R}^{m}$ $\theta y \in Q$

Então, as equaçõos em (3.31) tem a forma:

$$
\left\{\begin{array}{l}
\dot{x}=B x+\sum_{j=2}^{r \cdots 1} \frac{1}{j !} f_{j}^{1}(x, y, \alpha)+O\left(|(x, y, \alpha)|^{r}\right) \\
\frac{d y}{d t}=A_{Q^{\prime}} y+\sum_{j=2}^{r-1} \frac{1}{j !} f_{j}^{2}(x, y, \alpha)+O\left(|(x, y, \alpha)|^{r}\right)
\end{array}\right.
$$

onde

$$
\begin{aligned}
& f_{j}^{1}(x, y, \alpha)=\Psi(0)\left[j ! L_{j}(\alpha)(\Phi x+y)+F_{j}(\Phi x+y, \alpha)\right], \\
& f_{j}^{2}(x, y, \alpha)=(I-\pi) X_{0}\left[j ! L_{j}(\alpha)(\Phi x+y)+F_{j}(\Phi x+y, \alpha)\right] .
\end{aligned}
$$

Observando que a variável de dimensão finita é $(x, \alpha) \in \mathbb{R}^{m+p}$ e a variável de dimensão infinita é $y \in Q^{1}$, podemos aplicar o algoritmo da forma normal para equações diferenciais funcionais retardadas, donde obtemos que a forma normal para a equação (3.27) até o termo de ordem $r-1$, é dada por:

$$
\left\{\begin{array}{l}
\dot{x}=B x+\sum_{j=2}^{r-1} \frac{1}{j !} g_{j}^{1}(x, y, \alpha)+O\left(|(x, y, \alpha)|^{r}\right), \\
\frac{d y}{d t}=A_{Q^{1}} y+\sum_{j=2}^{r-1} \frac{1}{j !} g_{j}^{2}(x, y, \alpha)+O\left(|(x, y, \alpha)|^{r}\right),
\end{array}\right.
$$


(2) 111

$$
\begin{aligned}
& y_{j}^{l}(x, y, \alpha)=\left(I-I_{I m\left(M_{j}^{1}\right)}^{1}\right) \Psi(0)\left[j ! L_{j}(\alpha)(\Phi x+y)+F_{j}(\Phi x+y, \alpha)\right], \\
& y_{j}^{2}(x, y, \gamma)=\left(I \quad I_{I m\left(M_{j}^{2}\right)}^{2}\right)(I-\pi) X_{0 l}\left[j ! L_{j}(\alpha)(\Phi x+y)+F_{j}(\Phi x+y, \alpha)\right] .
\end{aligned}
$$

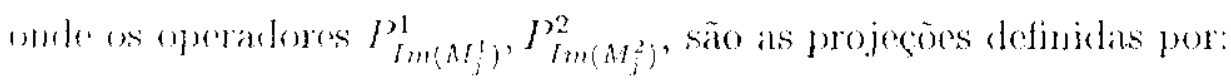

$$
\begin{array}{ll}
P_{\operatorname{lm}\left(M_{j}^{\prime}\right)}: & V_{j}^{m}\left(\mathbb{R}^{m}\right) \longrightarrow \operatorname{Im}\left(M_{j}^{1}\right), \\
I_{\ln \left(M_{j}^{2}\right)}^{2}: & V_{j}^{m}(K e r \pi) \longrightarrow \operatorname{Im}\left(M_{j}^{2}\right) .
\end{array}
$$

e o operador $M_{j}=\left(M_{j}^{1}, M_{j}^{2}\right)$ é definido por:

$$
\begin{aligned}
& M_{j}: V_{j}^{m+p}\left(\mathbb{R}^{m}\right) \times V_{j}^{m+p}(K \operatorname{er}(\pi)) \rightarrow V_{j}^{m+p}\left(\mathbb{R}^{m}\right) \times V_{j}^{m+p}(K e r(\pi)), \\
& \left(M_{j}^{1} p\right)(x, \alpha)=D_{x} p(x, \alpha) B x-B p(x, \alpha) \\
& \left(M_{j}^{2} h\right)(x, \alpha)=J_{x} h(x, \alpha) B x-A_{Q^{1}} h(x, \alpha),
\end{aligned}
$$

rom domínio $\left.D\left(M_{j}\right)=V_{j}^{m+p}\left(\mathbb{R}^{m}\right) \times V_{j}^{m+p}\left(Q^{l}\right)\right)$.

Agora obtemos, da teoria da Seção 3.4 , as condiçoes de näo-ressonaneia para equaçoes diferenciais funcionais retardadas com parâmetro.

Definição 3.6.1 Dizemos que a equação (3.27) safisfaz as condiçues de não-ressonância relativamente à $\Lambda \subset \sigma\left(A_{0}\right)$, se.

$$
(q: \lambda) \neq \mu \text { para todo } \mu \in \sigma\left(A_{0}\right) \backslash \Lambda, q=\left(q_{1}, \ldots, q_{n}\right) \in \mathbb{N}^{n} e \mid q_{1} \geq 2,
$$

onde $\lambda=\left(\lambda_{1}, \ldots, \lambda_{m}\right)$ e $\lambda_{1}, \ldots, \lambda_{m}$ sìo os elenentos de $\Lambda$, e cada um deles aparecem tantas nezes quanto sua multiplicidade como miz da equaçâ caracteristica dada por (3.3).

Finalmonte, temos um teorema, análogo no Teorema 3.4.2, resumindo a teoria para equaçoes diferenciais funcionais retardadas con um parâmetro.

Teorema 3.6.1 Consideremos a cquação (3.27) e a decomposição de C, dada por:

$$
C=P \oplus Q
$$

onde $P$ é o subespaco invariante pelo gerador infinitesimal $A_{0}$. associado ao subconjunto nào nazio e finito $\Lambda$ de autovalores. Assim, lemos a decomposiça $u=\Phi x+y$ onde $x \in \mathbb{R}^{m}, y \in Q$, dim $P=m e \Phi$ é urna base prara $P$. Se as condiçòes de nàoressonância relativamente a $\Lambda$ da Definiça 3.6 .1 são satisfertas, entào, existe uma mu-

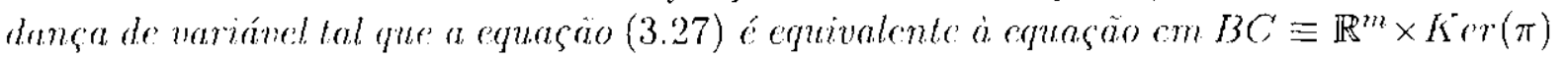

$$
\left\{\begin{aligned}
\dot{x} & =B x+\sum_{j=2}^{r-1} \frac{1}{j !} g_{j}^{1}(x, y, \alpha)+O\left(|(x, y, \alpha)|^{r}\right), \\
\frac{d y}{d t} & =A_{Q^{\prime}} y+\sum_{j=2}^{r \cdots 1} \frac{1}{j !} g_{j}^{2}(x, y, \alpha)+O\left(|(x, y, \alpha)|^{r}\right),
\end{aligned}\right.
$$


onde $g_{1}^{1} \cdot g_{j}^{2}$ siou obtidos como $\mathrm{cm}(3.36)$, com $g_{j}^{2}(x, 0)=0$, para $j>2$. F.s ca cquaçio está un forma normal relatinamente a $P$. Se existir uma variedade localmente inveriante para "rquaçio (3.27). tangrnte à $P$ no zcro, contäo, essa satisfaz $y=0$ e o fluro sobre eseat

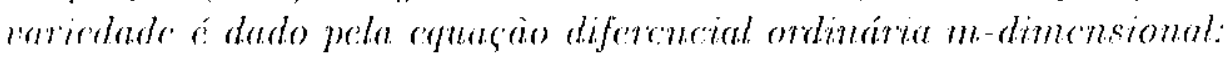

$$
x=B x+\sum_{j=1}^{r-1} \frac{1}{j !} g_{j}^{1}(x, y, \alpha)+O\left(\left\{\left.(x, y, \alpha)\right|^{r}\right),\right.
$$

"qual restá na forma normal, no sentido usual para aquacoes diferenciais ordinárins.

\subsection{Singularidade do tipo Bogdanov-Takens}

Nosta seção, faremos aplicaçōes à singularidade do tipo Bogdanov-Takens. O nosso estudo consistirá, numa situação geral, do cálculo da forma normal para equaçòes diforenciais funcionais retardadas escalares com singularidade do tipo Bogdanov-Takens.

$1^{\prime \prime} \Lambda_{\text {plicaçào }}$

Consideremos a equação diferencial funcional retardada escalar definida no espaço $C^{\prime}=C^{\prime}([-r, 0], \mathbb{R})$,

$$
\dot{z}(i)=L\left(z_{t}\right)+F\left(z_{t}\right),
$$

onde $L: C \rightarrow R$ c im operador linear limitado of $F \in C^{N}, N \geq 2$, definida de $C \mathrm{em} \mathbb{R}$. conl $F(0)=0$ : $D F^{\prime}(0)=0$.

Sejan $T(t), t \geq 0$, o $C_{0}$-semigrupo das soluções da equação linear:

$$
\dot{z}(t)=L\left(z_{t}\right),
$$

(: $A_{0}$ o gerador infinitesimal associado.

Suponhamos que a cquação característica $\Delta(\lambda)=\lambda-L\left(e^{\lambda}\right)$ :

(i) tenha $\lambda=0$ como antovalor de multiplicidade dois,

(ii) näo tenha neuhum autovalor, além de $\lambda=0$, com parte real zero.

Portanto, da hipótese (i.), temos:

$$
\Delta(0)=0, \quad \frac{\partial \Delta(0)}{\partial \lambda}=0 \quad \text { e } \frac{\partial^{2} \Delta(0)}{\partial \lambda^{2}} \neq 0
$$

ous scja,

$$
L(1)=0, \quad L(\theta)=1 \quad \text { e } L\left(\theta^{2}\right) \neq 0 .
$$

Consideremos a decomposiçäo de $C$ por $\Lambda=\{0\}$ dada por:

$$
C=P \oplus Q
$$

onde $P$ é o espaço invariante pelo gerador infinitesimal $A_{0}$ associado ao autovalor $\lambda=0$, darlo por:

$$
P=\operatorname{Ker}\left(A_{0}\right)^{k_{0}},
$$


Whin o ascondente de 0 . Um elomento $\phi \in P$ se, o somente se,

$$
\dot{\phi}, \ldots \phi^{\left(k_{0}-1\right)} \in D\left(A_{0}\right) e A_{0}^{k_{0} \phi} \equiv 0
$$

111 isijil.

$$
L\left(\phi^{\left(k_{1}+1\right)}\right)=\left(1 . L\left(\phi^{\left(k_{(1)}-2\right)}\right)=\phi^{\left(k_{0}-1\right)}(0), \ldots, L(\phi)=\dot{\phi}(0) e \phi^{\left(k_{(1)}\right)}=0 .\right.
$$

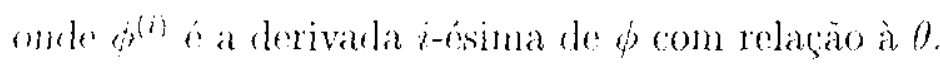

Entiào:

$$
\phi^{(i)}(\theta)=i ! a_{i}+(i+1) ! a_{i+1} \theta+\frac{(i+2) !}{2 !} a_{i+2} \theta^{2}+\cdots+\frac{\left(k_{0}-1\right) !}{\left(k_{0}-(i+1)\right) !} a_{k_{0}-1} \theta^{k_{0}-(i+1)}
$$

Usando as condigoes (3.39), consegumos:

- $L\left(\phi^{\left\{k_{0}-1\right\}}\right)=L\left(\left(k_{0}-1\right) ! a_{k_{0}-1}\right)=\left(k_{0}-1\right) ! a_{k_{0}-1} L(1)=0$

- $L\left(\phi^{\left(k_{0}-2\right)}\right)=\left(k_{0}-2\right) ! a_{k_{0}-2} L(1)+\left(k_{0}-1\right) ! a_{k_{0}-1} L(\theta) \stackrel{(3.41)}{=} \phi^{\left(k_{0}-1\right)}(0)=\left(k_{0}-1\right) ! a_{k_{0}-1}$.

- $L\left(\phi^{\left(k_{i 1}-3\right)}\right)=\left(k_{0}-3\right) ! a_{k_{0}-3} L(1)+\left(k_{0}-2\right) ! a_{k_{0}-2} L(\theta)+\frac{\left(k_{0}-1\right) ! a_{k_{0}-1}}{2 !} L\left(\theta^{2}\right)$

$$
\stackrel{(3.41)}{=} \phi^{\left(k_{i} \cdot 2\right)}(0)=\left(k_{0}-2\right) ! a_{k_{01}-2} \Longrightarrow a_{k_{0}} \quad 1=0
$$

- $L\left(\phi^{\left(k_{0}-4\right)}\right)=\left(k_{0}-4\right) ! a_{k_{0}-4} L(1)+\left(k_{0}-3\right) ! a_{k_{0}-3} L(\theta)+\frac{\left(k_{0}-2\right) ! a_{k_{0}-2}}{2 !} L\left(\theta^{2}\right)$

$$
\stackrel{(3.41)}{=} \phi \phi^{\left(k_{0}-2\right)}(0)=\left(k_{0}-2\right) ! a_{k_{0}-2} \Longrightarrow a_{k_{0}-2}=0
$$

- $L\left(\phi^{\left.\left(k_{0}\right) \cdot\left(k_{1}-1\right)\right)}\right)=L(\dot{\phi})=a_{1} L(1)+2 a_{2} L(\theta)+3 a_{3} L\left(\theta^{2}\right)=\phi(0) \stackrel{\langle 3.41\rangle}{=} \phi^{(2)}(0)=2 a_{2}$

$$
\rightarrow a_{3}=0
$$

- $L(\phi)=a_{0} L(1)+a_{1} L(\theta)+a_{2} L\left(\theta^{2}\right) \stackrel{(3.41)}{=} \dot{\phi}(0)=a_{1} \Longrightarrow a_{2}=0$,

oli seja, $a_{k_{0}-1}=\cdots=a_{2}=0$ e $a_{0}, a_{1}$ säo reais quaisquer.

Portanto, o espaço $P$ tem ascendente dois e, é dado por:

$$
P=\operatorname{Ker}\left(A_{0}\right)^{2}=\left\{\phi \in D\left(A_{0}\right): \phi(\theta)=a_{0}+a_{1} \theta, \quad \text { e } a_{0}, a_{1} \in \mathbb{R}\right\} .
$$

Assim, podemos tomar $\Phi=\left\{\phi_{1}(\theta), \phi_{2}(\theta)\right\}, \theta \in[-r, 0]$, como sendo uma base para $P$, com:

$$
\phi_{1}(\theta)=1, \phi_{2}(\theta)=\theta, \text { para } \theta \in[-r, 0]
$$


(omo $\lambda-0$ tem aserendente dois, temos que:

$$
\rho^{T}=\operatorname{Kr}\left(A_{0}^{T}\right)^{2}=\left\{b \in D\left(A_{0}^{T}\right): \psi(s)=b_{0} \cdots s b_{1}, e b_{0}, b_{1} \in \mathbb{R}\right\} .
$$

"intan. tomenos a base $\Psi=\operatorname{col}\left\{\tilde{\psi}_{1} \cdot \tilde{\psi}_{2}\right\}$ de: $P^{T}$, onde

$$
\tilde{\psi}_{1}(s)=\psi_{1}(0)-s \psi_{2}(0) \text { e } \tilde{\psi}_{2}(s)=\psi_{2}(0)
$$

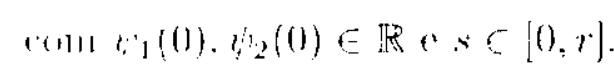

P’ara cque possamos ter $(\Psi, \Phi)=I$, é necessário e suficiente escolhermos $\psi_{1}(0), \psi_{2}(0)$ reais, tais que:

$$
\left\{\begin{array}{l}
\left(\psi_{1}(0)-s \psi_{2}(0), 1\right)=1 \\
\left(\psi_{1}(0)-s \psi_{2}(0), \theta\right)=0
\end{array}\right.
$$

oll seja,

$$
\left\{\begin{array}{l}
\psi_{1}(0)-\int_{-r}^{0} \int_{0}^{\theta}\left(\psi_{1}(0)-(\xi-\theta) \psi_{2}(0)\right) d \eta(\theta) d \xi=1 \\
-\int_{-r}^{\prime \prime} \int_{0}^{\theta}\left(h_{1}(0)-(\xi-\theta) \psi_{2}(0)\right) d \eta(\theta) \xi d \xi=0,
\end{array}\right.
$$

o gue implica.

$$
\left\{\begin{array}{l}
\psi_{1}(0)-\psi_{1}(0) L(0)+\psi_{2}(0) L\left(\frac{\theta^{2}}{2}\right)-\psi_{2}(0) L\left(0^{2}\right)=1 \\
-\psi_{1}(0) L\left(\frac{\theta^{2}}{2}\right)-\psi_{2}(0) L\left(\frac{\theta^{3}}{3 !}\right)=0
\end{array}\right.
$$

r isatudo (3.39), obtemos:

$$
\left\{\begin{array}{l}
\psi_{2}(0)=-L\left(\frac{\theta^{2}}{2}\right)^{-1} \\
\psi_{1}(0)=L\left(\frac{\theta^{2}}{2}\right)^{-2} L\left(\frac{\theta^{3}}{3 !}\right)
\end{array}\right.
$$

Concluindo, temos que:

$$
\begin{array}{ll}
P=\operatorname{grt} \Phi, \quad \Phi(\theta)=(1, \theta), & \text { para } \theta \in[-r, 0], \\
P^{T}-\operatorname{ger} \Psi, \quad \Psi(s)=\operatorname{col}\left(\psi_{1}(0)-s \psi_{2}(0), \psi_{2}(0)\right), & \text { para } s \in[0, r] .
\end{array}
$$

onde $\psi_{1}(0)$ e $\psi_{2}(0)$ são conno em $(3.42)$.

A matriz $B$ satisfazendo $\dot{\Phi}=\Phi B$ e $-\dot{\Psi}=B \Psi$ é dada por:

$$
B=\left[\begin{array}{ll}
0 & 1 \\
0 & 0
\end{array}\right] \text {. }
$$


Observemos que o ímico antovalor de $B$ e $\lambda=0$, e esse tem multiplicidarle dosis. rntar. a equaça diferencial ordinária bidinensional sobre a variedade central tom uma simgularidade do tipo Bogdtanov-'Takens.

(alcularenes a forma normal para a equação diferencial ordinária bidinensional sobre a varieclide central alé os termos de orden dois.

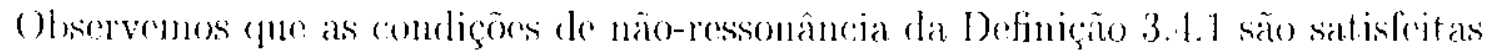

Do fate:

Pela hipótese (ii), a parte real de we diferente de zero para todo $\mu \in \sigma\left(A_{0}\right) \backslash A$. entao. para $q=\left(q_{1}, q_{2}\right)$ pertencente a $\mathbb{N}^{2}$, com $|q|=j, j \geq 2$ e $\lambda=(0.0)$, conseguimos:

$$
(q, \dot{\lambda})-\mu=-\mu \neq 0 \text {. }
$$

Agora, descreveremos a forma normal sobre a variedade central como foi desenvolvido naks seçòes anterioress.

Consideremos a expausio de Taylor para $F$ até os termos de ordem dois.

$$
F(u)=\frac{1}{2 !} F_{2}(u)+O\left(|u|^{3}\right)
$$

"x) $n \in C$ !

Pela decomposigão de $C$ por $\Lambda$, como em (3.40), podemos deconpor u $E$ C. da loma:

$$
u=\Phi x+y,
$$

$\Leftrightarrow m \in \mathbb{R}^{m}$ e $y \in Q$.

Definimos $f_{2}(x, y)$ como cm $(3.11)$,

$$
f_{2}(x, y)=\left[\begin{array}{c}
\psi_{1}(0) \\
\psi_{2}(0) \\
(I-\pi) X_{0}
\end{array}\right] F_{2}(\Phi x+y)
$$

onde as duas primeiras linhas representam a componente de $f_{2}(x, y)$ em $\mathbb{R}^{2}$ e a terceira linha representa a componente de $f_{2}(x, y) \mathrm{em} \operatorname{Ker}(\pi)$.

() operador

$$
M_{j}^{1}: V_{j}^{2}\left(\mathbb{R}^{2}\right) \rightarrow V_{j}^{2}\left(\mathbb{R}^{2}\right)
$$

para $j \geq 2$, r dado por:

$$
M_{j}^{1}(p(x))=\left[\begin{array}{l}
x_{2} \frac{\partial p_{1}(x)}{\partial x_{1}}-p_{2}(x) \\
x_{2} \frac{\partial p_{2}(x)}{\partial x_{1}}
\end{array}\right]
$$

'om $x=\left(\begin{array}{l}x_{1} \\ x_{2}\end{array}\right) \in \mathbb{R}^{2}$ e $p(x)=\left(\begin{array}{l}p_{1}(x) \\ p_{2}(x)\end{array}\right) \in V_{j}^{2}\left(\mathbb{R}^{2}\right)$.

Agora, calcularemos o espaço complementar de $\operatorname{Im}\left(M_{2}^{1}\right)$ em $V_{2}^{2}\left(\mathbb{R}^{2}\right)$, para que possamos determinar os termos de ordom dois que nào podem ser eliminados da forma normal. 
(onsiderando at hase canônica de $V_{2}^{2}\left(\mathbb{R}^{2}\right)$.

$$
\left(\begin{array}{c}
x_{1}^{2} \\
0
\end{array}\right),\left(\begin{array}{c}
x_{1} x_{2} \\
0
\end{array}\right),\left(\begin{array}{c}
x_{2}^{2} \\
0
\end{array}\right),\left(\begin{array}{c}
0 \\
x_{1}^{2}
\end{array}\right),\left(\begin{array}{c}
0 \\
x_{1} x_{2}
\end{array}\right) \cdot\left(\begin{array}{c}
0 \\
x_{2}^{2}
\end{array}\right) \text {. }
$$

ention. suas respectivas imagens sob o operador $M_{2}^{1}$, são:

$$
\left(\begin{array}{c}
2 x_{1} x_{2} \\
0
\end{array}\right),\left(\begin{array}{c}
x_{2}^{2} \\
0
\end{array}\right),\left(\begin{array}{l}
0 \\
0
\end{array}\right) \cdot\left(\begin{array}{c}
-x_{1}^{2} \\
2 x_{1} x_{2}
\end{array}\right),\left(\begin{array}{c}
-x_{1} x_{2} \\
x_{2}^{2}
\end{array}\right),\left(\begin{array}{c}
\cdots x_{2}^{2} \\
0
\end{array}\right) \cdot
$$

Portanto, temos que:

$$
\operatorname{Im}\left(M_{2}^{1}\right)=\operatorname{ger}\left\{\left(\begin{array}{c}
2 x_{1} x_{2} \\
0
\end{array}\right),\left(\begin{array}{c}
x_{2}^{2} \\
0
\end{array}\right),\left(\begin{array}{c}
-x_{1}^{2} \\
2 x_{1} x_{2}
\end{array}\right),\left(\begin{array}{c}
-x_{1} x_{2} \\
x_{2}^{2}
\end{array}\right)\right\} .
$$

I)aí, como os vetores,

$$
\left(\begin{array}{c}
2 x_{1} x_{2} \\
0
\end{array}\right),\left(\begin{array}{c}
x_{2}^{2} \\
0
\end{array}\right),\left(\begin{array}{c}
-x_{1}^{2} \\
2 x_{1} x_{2}
\end{array}\right),\left(\begin{array}{c}
-x_{1} x_{2} \\
x_{2}^{2}
\end{array}\right),\left(\begin{array}{c}
0 \\
x_{1} x_{2}
\end{array}\right),\left(\begin{array}{c}
0 \\
x_{1}^{2}
\end{array}\right) .
$$

säto linearmente independentes, podemos escolher o espaço complementar de $\operatorname{Im}\left(M_{2}^{1}\right)$ em $V_{2}^{2}\left(\mathbb{R}^{2}\right)$ como:

$$
\operatorname{Im}\left(M_{2}^{1}\right)^{c}=\operatorname{ger}\left\{\left(\begin{array}{c}
0 \\
x_{1}^{2}
\end{array}\right),\left(\begin{array}{c}
0 \\
x_{1} x_{2}
\end{array}\right)\right\} .
$$

Tomemos $\alpha, \beta, \gamma \in \mathbb{R}$, tais que:

$$
F_{2}(\Phi x)=\alpha x x_{1}^{2}+\beta x_{1} x_{2}+\gamma x_{2}^{2},
$$

(nit.îo,

$$
\begin{aligned}
f_{2}^{1}(x, 0)= & \Psi(0) F_{2}(\Phi x)=\left(\begin{array}{c}
\psi_{1}(0) \\
\psi_{2}(0)
\end{array}\right) \alpha x_{1}^{2}+\beta x_{1} x_{2}+\gamma x_{2}^{2} \\
=: & {\left[-\alpha \psi_{1}(0)\left(\begin{array}{c}
-x_{1}^{2} \\
2 x_{1} x_{2}(0)
\end{array}\right)+\frac{\beta}{2} \psi_{1}(0)\left(\begin{array}{c}
2 x_{1} x_{2} \\
0
\end{array}\right)+\gamma \psi_{1}(0)\left(\begin{array}{c}
x_{2}^{2} \\
0
\end{array}\right)+\right.} \\
& \left.\frac{\gamma}{2} \psi_{2}(0)\left(\begin{array}{c}
0 \\
2 x_{2}^{2}(0)
\end{array}\right)\right]+\left[\left(2 \alpha \psi_{1}(0)+\beta \psi_{2}(0)\right)\left(\begin{array}{c}
0 \\
x_{1} x_{2}
\end{array}\right)+\alpha \psi_{2}(0)\left(\begin{array}{c}
0 \\
x_{1}^{2}
\end{array}\right)\right]
\end{aligned}
$$

Desse modo, temos que:

$$
\left(I-\Gamma_{I, 2}^{1}\right) f_{2}^{1}(x, 0)=\alpha \psi_{2}(0)\left(\begin{array}{c}
0 \\
x_{1}^{2}
\end{array}\right)+\left(2 \alpha \psi_{1}(0)+\beta \psi_{2}(0)\right\}\left(\begin{array}{c}
0 \\
x_{1} x_{2}
\end{array}\right)
$$

onde $P_{t, 2}^{1}$ é a primeira componente da projeção:

$$
P_{I, 2}: V_{2}^{2}\left(\mathbb{R}^{2}\right) \times V_{2}^{2}(\operatorname{Ker}(\pi)) \rightarrow \operatorname{Im}\left(M_{2}^{1}\right) \times \operatorname{Im}\left(M_{2}^{2}\right),
$$

$\operatorname{com} P_{I, 2}=\left(P_{I, 2}^{1}, P_{I, 2}^{2}\right)$.

Portanto, o fluxo sobre a variedade central é dado na forma normal até os termos de 
scymela ordent, por:

$$
\left\{\begin{array}{l}
\dot{x}_{1}=x_{2}+O\left(|x|^{3}\right) \\
\dot{x}_{2}=a x_{1}^{2}+b x_{1} x_{2}+O\left(|x|^{3}\right)
\end{array}\right.
$$

alide

$$
\begin{gathered}
a=\frac{\alpha}{2} \psi_{2}(0)=-\alpha L\left(\theta^{2}\right) \\
b=\alpha \psi^{\prime}{ }_{1}(0)+\frac{\beta}{2} \psi_{2}(0)=\frac{2}{3} \alpha L\left(\theta^{2}\right)^{-2} L\left(\theta^{3}\right)-\beta L\left(\theta^{2}\right)^{-1}
\end{gathered}
$$

sio constantes reais.

De modo geral, $a b \neq 0$, e as equaçoes em $(3.44)$ determinam unal versio da singularidade do tipo Bogdanov-Takens e no caso em que $a b=0$, precisanos calcular os termos de ordens superiores para a forma normal, ver $[2,3,10]$.

Sabemos de $[2,3,10]$, que a versão geral da singularidade do tipo Bogdanov-Takens na forma normal sobre a variedade central, com $a b \neq 0$, é dada por:

$$
\left\{\begin{array}{l}
\dot{x}_{1}=x_{2}+O\left((|\lambda|+|x|)|x|^{2}\right) \\
\dot{x}_{2}=\lambda_{1} x_{1}+\lambda_{2} x_{2}+a x_{1}^{2}+b x_{1} x_{2}+O\left((|\lambda|+|x|)|x|^{2}\right) .
\end{array}\right.
$$

Agora. queremos deterninar a versão geral da equaça diferencial funcional retardata escalar original com a singularidade do tipo Bogdanov-Takens. Para isso, tomemos mma perturbação da equação (liferencial funcional retardada original (3.38), da forma:

$$
v_{1} z(t)-v_{2} z(t-r), \operatorname{con} v_{1}, v_{2} \in \mathbb{R}
$$

donde obtemos:

$$
\dot{z}(l)=v_{1} z(t)-v_{2} z(t-r)+L(z(t))+F(z(t)) .
$$

Consideremos a decomunosição de $B C$,

$$
B C=P \oplus \operatorname{Ker}(\pi),
$$

onde $P$ é dado cono em (3.40).

Como

$$
z_{t}(\theta)=\Phi(\theta) x+y(\theta), \theta \in[-r, 0]
$$

$\operatorname{com} \Phi(\theta)=(1, \theta), x \in \mathbb{R}^{m}, y \in Q$ e usando as equações em (3.10), obtemos:

$$
\left\{\begin{array}{l}
\dot{x}=B x+\Psi(0)\left[F(\Phi x+y)+v_{1}(\Phi(0) x+y(0))-v_{2}(\Phi(-r) x+y(-r))\right] \\
\frac{d y}{d t}=A_{Q^{\prime}} y+(I-\pi) X_{0}\left[F(\Phi x+y)+v_{1}(\Phi(0) x+y(0))-v_{2}(\Phi(-r) x+y(-r))\right],
\end{array}\right.
$$

Donde obtemos,

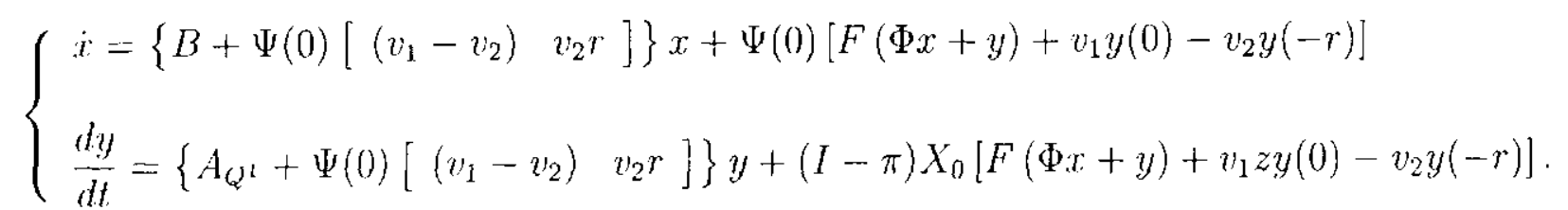


Pana determinarmos a versão geral da egluagăo diferencial funcional retardada escalar original com a singularidade do tijo Bogdanov-Takens, cuja equação na forma normal

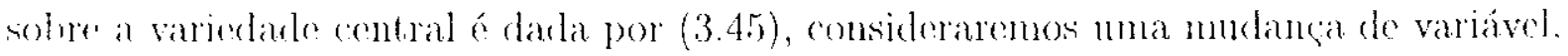

$$
x \mapsto D x,
$$

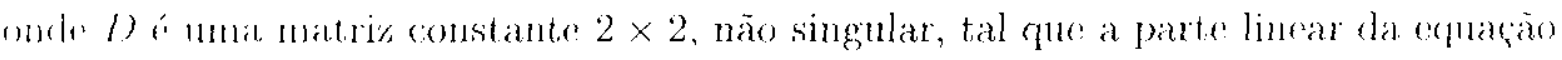

$$
r-\left\{B+\psi(0)\left[\begin{array}{ll}
\left(v_{1}-v_{2}\right) & v_{2} r
\end{array}\right]\right\} x+\Psi(0)\left[F(\Phi x+y)+v_{1} y(0)-v_{2} y(-r)\right],
$$

seja 'levala' na parte linear do sistema (3.45), ou seja,

$$
\begin{aligned}
D^{-1}\left[B+\Psi(0)\left[\left(v_{1}-v_{2}\right) v_{2} r\right]\right] D & =B+\left[\begin{array}{c}
0 \\
\psi_{2}(0)
\end{array}\right]\left[\begin{array}{ll}
\lambda_{1} \psi_{2}(0) \cdot 1 & \lambda_{2} \psi_{2}(0)^{-1}
\end{array}\right] \\
& =\left[\begin{array}{cc}
0 & 1 \\
\lambda_{1} & \lambda_{2}
\end{array}\right],
\end{aligned}
$$

o que equivale à:

$$
\left\{\begin{array}{l}
D^{-1} B D=B \\
D^{-1} \Psi(0)=\left[\begin{array}{c}
0 \\
\psi_{2}(0)
\end{array}\right]
\end{array}\right.
$$

e portinito, olttemos:

$$
D=\left[\begin{array}{cc}
1 & \psi_{1}(0) \psi_{2}(0)^{-1} \\
0 & 1
\end{array}\right]
$$

Fazondo essa mudança de variável com $y=0$, temos que:

$$
\begin{aligned}
& D^{-1}\left[B x+\Psi(0)\left[\left(v_{1}-v_{2}\right) \quad v_{2} r\right]\right] D x=D^{-1} B D x+D^{-1} \Psi(0)\left[\left(v_{1}-v_{2}\right) v_{2} r\right] D x \\
& =B . x+\left[\begin{array}{c}
0 \\
\psi_{2}(0)
\end{array}\right]\left[\begin{array}{ll}
\left(v_{1}-v_{2}\right) & v_{2} r
\end{array}\right] D x=B x+\left[\begin{array}{c}
0 \\
\psi_{2}(0)
\end{array}\right]\left[\begin{array}{ll}
\lambda_{1} \psi_{2}(0)^{-1} & \lambda_{2} \psi_{2}(0)^{-1}
\end{array}\right] x \\
& =\left[\begin{array}{c}
x_{2} \\
\lambda_{1} x_{1}+\lambda_{2} x_{2}
\end{array}\right]
\end{aligned}
$$

onde

$$
\lambda_{1}=\left(v_{2}-v_{1}\right) \frac{2}{L\left(\theta^{2}\right)} e \lambda_{2}=\left[\left(v_{1}-v_{2}\right) \frac{2}{3} \frac{L\left(\theta^{3}\right)}{L\left(\theta^{2}\right)^{2}}-v_{2} \frac{2 r}{L\left(\theta^{2}\right)}\right] .
$$

Consideremos a mudança de variável que, para $y=0$, 'transforma' a equação

$$
\dot{x}=B x+\frac{1}{2} \Psi(0) F_{2}(x, 0)+O\left(|x|^{3}\right)
$$


('II) slla forma normal:

$$
\left\{\begin{array}{l}
x_{1}=x_{2}+O\left(|x|^{3}\right) \\
x_{2}=a x_{1}^{2}+b x_{1} x_{2}+O\left(|x|^{3}\right)
\end{array}\right.
$$

diatia por:

$$
x \longmapsto x+\frac{1}{2} y_{2}^{1}(x)
$$

(unl $l l_{2}^{1}(x)=\left(\begin{array}{l}p_{1}(x) \\ p_{2}(x)\end{array}\right)$

Supoudo que

$$
F_{2}(x, 0)-\alpha x_{1}^{2}+\beta x_{1} x_{2}+\gamma x_{2}^{2}
$$

e fazendo a mudança de variável $x: D x$, para $y=0$, na equação

$$
\dot{x}=\left[B+\Psi(0)\left[\left(v_{1}-v_{2}\right) v_{2} r\right]\right] x+\frac{1}{2} \Psi(0) F_{2}(x, 0)+O\left(|x|^{3}\right),
$$

obtemos

$$
\begin{aligned}
x= & {\left[B+\left[\begin{array}{c}
0 \\
\psi_{2}(0)
\end{array}\right]\left[\begin{array}{ll}
\lambda_{1} \psi_{2}(0)^{-1} & \lambda_{2} \psi_{2}(0)^{-1}
\end{array}\right]\right] x+} \\
& +\frac{1}{2} \Psi(0) F_{2}(x, 0)-\frac{1}{2}\left[\begin{array}{c}
\psi_{1}(0) \\
0
\end{array}\right] F_{2}(x, 0)+\frac{1}{2}\left[\begin{array}{c}
0 \\
\psi_{2}(0)
\end{array}\right] . \\
& {\left[2 \alpha x_{1} x_{2} \psi_{1}(0) \psi_{2}(0)^{-1}+\alpha x_{2}^{2} \psi_{1}(0)^{2} \psi_{2}(0)^{-2}+\beta x_{2}^{2} \psi_{1}(0) \psi_{2}(0)^{-1}\right]+O\left(|x|^{3}\right) . }
\end{aligned}
$$

Agora, fazendo a mudança de variável

$$
x \longmapsto x+\frac{1}{2} U_{2}^{1}(x)
$$

$$
\begin{aligned}
& \text { coln } U_{2}^{1}(x)=\left(\begin{array}{l}
p_{1}(x) \\
p_{2}(x)
\end{array}\right), \text { conseguimos: } \\
& \left(I+D_{x} U_{2}^{1}(x)\right) \dot{x}=\left[B+\left[\begin{array}{c}
0 \\
\psi_{2}(0)
\end{array}\right]\left[\begin{array}{ll}
\lambda_{1} \psi_{2}(0)^{-1} & \lambda_{2} \psi_{2}(0)^{-1}
\end{array}\right] x+\frac{1}{2}[B+\right. \\
& \left.+\left[\begin{array}{c}
0 \\
\psi_{2}(0)
\end{array}\right] \cdot\left[\begin{array}{ll}
\lambda_{1} \psi_{2}(0)^{-1} & \lambda_{2} \psi_{2}(0)^{-1}
\end{array}\right]\right] U_{2}^{1}(x)+\Psi(0) F_{2}\left(x+\frac{1}{2} U_{2}^{1}(x), 0\right)- \\
& -\frac{1}{2}\left[\begin{array}{c}
\psi_{1}(0) \\
0
\end{array}\right] F_{2}\left(x+\frac{1}{2} U_{2}^{1}(x), 0\right)+\frac{1}{2}\left[\begin{array}{c}
0 \\
\psi_{2}(0)
\end{array}\right]\left[2 \alpha\left(x_{1}+\frac{1}{2} p_{1}(x)\right)\right. \\
& \left(x_{2}+\frac{1}{2} p_{2}(x)\right) \cdot \psi_{1}(0) \psi_{2}(0)^{-1}+\alpha\left(x_{1}+\frac{1}{2} p_{1}(x)\right)^{2} \psi_{1}(0)^{2} \psi_{2}(0)^{-2}+ \\
& \left.+\beta\left(x_{2}+\frac{1}{2} p_{2}(x)\right)^{2} \psi_{1}(0) \psi_{2}(0)^{-1}\right]+O\left(|x|^{3}\right) .
\end{aligned}
$$


Toimedo as explaçoes (3.16), (3.17) o as relaçoes:

$$
\left\{\begin{array}{l}
\psi_{1}(0)=\lambda_{2}\left(v_{1}-v_{2}\right)^{-1}-\lambda_{1} v_{2} r\left(v_{1}-v_{2}\right)^{-2}, \\
\psi_{2}(0)=\lambda_{1}\left(v_{1}-v_{2}\right)^{-1}
\end{array}\right.
$$

Mltrmos.

$$
\left\{\begin{array}{l}
x_{1}=x_{2}+O\left((|\lambda|+|x|)|x|^{2}\right) \\
x_{2}=\lambda_{1} x_{1}+\lambda_{2} x_{2}+a x_{1}^{2}+b x_{1} x_{2}+O\left((|\lambda|+|x|) \mid x_{1}^{2}\right) .
\end{array}\right.
$$

(com a, b c $\lambda=\left(\lambda_{1}, \lambda_{2}\right)$ daxtos como em (3.44) e $(3.46)$.

Conchúmos assim, que a versão geral para a equação diferencial funcional (3.38) com singularidade de Bogdanov-'Takens no zero é dada por:

$$
\dot{z}(t)=v_{1} z(t)-v_{2} z(t-r)+L\left(z_{t}\right)+F\left(z_{t}\right),
$$

onde $u_{1}, y_{2}$ säo reais.

$2^{\prime}$ Aplicagano

Agora, farrmos um caso particular da equação (3.38),

$$
\dot{z}(t)=\int(z(t), z(t-1)),
$$

(onu $f \in C^{N}\left(\mathbb{R}^{2}, \mathbb{R}\right), N \geq 2, f(0,0)=0$, e suponhamos que a equação característica $(3.3):$

(i) tenla $\lambda=0$ como autovalor de multiplicidade dois,

(ii) não tenha nouhum autovalor, diferente de zero, com partereal zero.

Podemos escrever a equação (3.48) da forma:

$$
\begin{aligned}
\dot{z}(t)= & z(t)-z(t-1)+\frac{1}{2}\left[A_{(2,0)} z^{2}(t)+A_{(1,1)} z(t) z(t-1)+A_{(0,2)} z^{2}(t-1)\right]+ \\
& +K(z(t), z(t-1)),
\end{aligned}
$$

onde $K(0,0)=0, D K(0,0)=0$ c $D^{2} K(0,0)=0$.

De fato:

Tomando a expansāo de Taylor para a equação (3.48), podemos escrever:

$$
\begin{aligned}
\dot{z}(t)= & a \tilde{z}(t)+b z(t-1)+\frac{1}{2}\left[A_{(2,0)} z^{2}(t)+A_{\langle 1,1)} z(t) z(t-1)+A_{(0,2)} z^{2}(t-1)\right]+ \\
& +K(z(t), z(t-1)) .
\end{aligned}
$$

Definimos os operadores $L: C \rightarrow \mathbb{R}$ e $F_{2}: C \rightarrow \mathbb{R}$, por:

$$
\begin{aligned}
L(\phi) & =a \phi(0)+b \phi(-1) \\
F_{2}(\phi) & =\left[A_{(2,0)} \phi^{2}(0)+A_{(1,1)} \phi(0) \phi(-1)+A_{(0,2)} \phi^{2}(-1)\right] .
\end{aligned}
$$


("ntan. pelas relagios em (3.39). temos que $a=-b=1$.

Das rerlaçós

$$
\left\{\begin{array}{l}
\psi_{1}(\theta)=L\left(\frac{\theta^{2}}{2}\right)^{-2} L\left(\frac{\theta^{3}}{3 !}\right) \\
\psi_{2}(0)=-L\left(\frac{\theta^{2}}{2}\right)^{-1},
\end{array}\right.
$$

olilomosi

$$
\left\{\begin{array}{l}
\psi_{1}(0)=\frac{2}{3} \\
\psi_{2}(0)=2
\end{array}\right.
$$

Consideremos a decomposição de $C$ por $A=\{0\}$, ou seja, $C=P \notin Q$, desise modo podenos escrever $z_{t} \in C$ como: $z_{t}=\Phi: t+y$.

Daí, para $y=0$, consmgumos:

$$
\begin{aligned}
F_{2}(\Phi x) & =\left[A_{(2,0)} \phi^{2}(0) x+A_{(1,1)} \phi(0) x \phi(-1) x+A_{(0,2)} \phi^{2}(-1) x\right] \\
& =\left[A_{(2,0)} x_{1}^{2}+A_{(1,1)} x_{1}\left(x_{1}-x_{2}\right)+A_{(0,2)}\left(x_{1}-x_{2}\right)^{2}\right] \\
& =\left[\left(A_{(2,0)}+A_{(1,1)}+A_{(0,2)}\right) x_{1}^{2}+\left(-A_{(1,1)}-2 A_{(0,2,)}\right) x_{1} x_{2}+A_{(0,2)} x_{2}^{2}\right] .
\end{aligned}
$$

Portanto, temos de (3.44), que o fluxo sobre a variedade central é dado na forma normal por:

$$
\left\{\begin{array}{l}
\dot{x}_{1}=x_{2}+O\left(|x|^{3}\right) \\
\dot{x}_{2}=a x_{1}^{2}+b x_{1} x_{2}+O\left(|x|^{3}\right),
\end{array}\right.
$$

onde

$$
a=-\alpha I_{L}\left(\theta^{2}\right)=\left(A_{(2,0)}+A_{(1,1)}+A_{(0,2)}\right)
$$

$$
b=\frac{2}{3} \alpha L\left(\theta^{2}\right)^{-2} L\left(\theta^{3}\right)-\beta L\left(\theta^{2}\right)^{-1}=\frac{1}{3}\left(2 A_{(2,0)}-A_{(1,1)}-4 A_{(0.2)}\right)
$$

Obtemos de (3.47) que a versão geral para a equaçăo diferancial retardada (3.48) com a singularidade do tipo Bogdanov-Takens, é da forma:

$$
\dot{z}(t)=v_{1} z(t)-v_{2} z(t-1)+z(t)-z(t-1)+\frac{1}{2} F_{2}(z(t), z(t-1))+K(z(t), z(t-1)),
$$

oll seja,

$$
\begin{aligned}
\dot{z}(1)= & \left(1+\eta_{1}\right) z(t)-\left(1+v_{2}\right) z(t-1)+\frac{1}{2}\left[A_{(2,0)} z^{2}(t)+A_{(1,1)} z(t) z(t-1)+\right. \\
& \left.+A_{(0,2)} z^{2}(t-1)\right]+K(z(t), z(t-1)),
\end{aligned}
$$

rom $K(0,0)=0, D K(0,0)=0$ с $D^{2} K(0,0)=0$

Conchuinos que a versão geral da forma nomal sobre a variedarle central, com $a b \neq 0$, 
$\therefore$ diatia pres:

$$
\left\{\begin{array}{l}
x_{1}=x_{2}+O\left((|\lambda|+|x|)|x|^{2}\right) \\
i_{2}=\lambda_{1} x_{1}+\lambda_{2} x_{2}+a x_{1}^{2}+b x_{1} x_{2}+O\left((|\lambda|+|x|)|x|^{2}\right),
\end{array}\right.
$$

(1) mik.

$$
\left\{\begin{array}{l}
\lambda_{1}=2\left(u_{1}-v_{2}\right) \\
\lambda_{2}=\frac{2}{3}\left(u_{1}+2 v_{2}\right) \\
a=\left(A_{(2,0)}+A_{(1,1)}+A_{(0,2)}\right) \\
b=\frac{1}{3}\left(2 A_{(2,0)}-A_{(1,1)}-4 A_{(0,2)}\right)
\end{array}\right.
$$

\section{Observação:}

$\operatorname{Lim}[2,3,10]$, considerando eguações diferenciais ordinárias há uma descrição dos retratos de fase numa vizinhança da origem para a versão geral. Esse resultado pode ser ut ilizado para (3.50) atravós dos coeficientes considerados acina, então podemos usar os coeficientes $a, b, c^{2}$ os parannetros $\lambda_{1}, \lambda_{2}$, que surgen na versào geral da forma normal para descrever o possível retrato de lase em tomos dos coeficientes da equacão diferencial funcional retadada original sem a necessidade de calcularmos previamente a variedade? central. Em particular, a estabilidade das órbitas homoclínicas e periódicas ocorrem para rertes valores dos parâmetros e dependem do sinal do produto:

$$
\left(A_{(2,0)}+A_{(1,1)}+A_{(0,2)}\right)\left(2 A_{(2,0)}-A_{(1,1)}-4 A_{(0,2)}\right) .
$$

$3^{n}$ Aplicação

() nosso objetivo a ilustrar o cálculo da forma normal para termos de ordem maiores que dois, vamos mostrar também como esse procedimento difere do caso de dimensão finita. Consideraremos um caso particular da equação (3.48), cuja condição $a . b \neq 0$ não está satisfeita,

$$
\dot{z}(t)=z(t)-z(t-1)+\alpha z(t)[z(l)-z(t-1)]+\beta z(l) z^{2}(t-1),
$$

$\operatorname{com} \alpha, \beta \in \mathbb{R}$.

Nesse caso, obtemos de (3.44), que os coeficientes da forma normal sobre a variedade central são:

$$
\left\{\begin{array}{l}
a=\left(A_{(2,0)}+A_{(1,1)}+A_{(0,2)}\right)=2 \alpha-2 \alpha+0=0 \\
b=\frac{1}{3}\left(2 A_{(2,0)}-A_{(1,1)}-4 A_{(0,2)}\right)=\frac{1}{3}(2(2 \alpha)+2 \alpha+0)=2 \alpha
\end{array}\right.
$$

Como essa equação é um caso particular da equação (3.48), o autovalor $\lambda=0$ tem multiplicidade dois e nenhum outro autovalor tem parte real zero. As condições de nãoressonância são satisfeitas e implicam que $\operatorname{Im}\left(M_{j}^{2}\right)=V_{j}^{2}(\operatorname{Ker}(\pi)), j \geq 2$, portanto, 
pertrumos tomar a projoçion

$$
I_{1, j}^{2}: V_{j}^{2}(\operatorname{Sin}(\pi))-+V_{j}^{2}(\operatorname{lic}(\pi))
$$

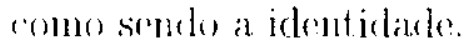

Agora. calcularemos a mudança de variavel $l_{2}(x)$, dada cm (3.21). Para isso. preci-

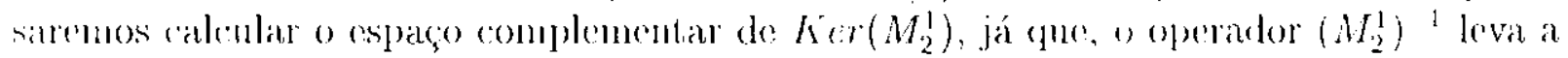
indagem de operater $M_{2}^{1}$ sobre $\left(\operatorname{Kor}\left(M_{2}^{1}\right)\right)^{r}$.

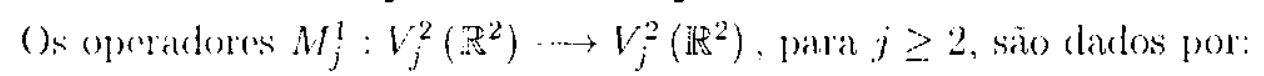

$$
M_{j}^{1}(p(x))=\left[\begin{array}{l}
x_{2} \frac{\partial p_{1}(x)}{\partial x_{1}}-p_{2}(x) \\
x_{2} \frac{\partial p_{2}(x)}{\partial x_{1}}
\end{array}\right] .
$$

Comsiderando a base canônica do $V_{2}^{2}\left(\mathbb{R}^{2}\right)$,

$$
\left(\begin{array}{c}
x_{1}^{2} \\
0
\end{array}\right),\left(\begin{array}{c}
x_{1} x_{2} \\
0
\end{array}\right),\left(\begin{array}{c}
x_{2}^{2} \\
0
\end{array}\right),\left(\begin{array}{c}
0 \\
x_{1}^{2}
\end{array}\right),\left(\begin{array}{c}
0 \\
x_{1} x_{2}
\end{array}\right),\left(\begin{array}{c}
0 \\
x_{2}^{2}
\end{array}\right) .
$$

podemos cescrever $p(x) \in V_{2}^{2}\left(\mathbb{R}^{2}\right)$ da forma:

$$
p(x)=\left(\begin{array}{l}
p_{1}(x) \\
p_{2}(x)
\end{array}\right)=\left(\begin{array}{l}
a_{1} x_{1}^{2}+a_{2} x_{1} x_{2}+a_{3} x_{2}^{2} \\
a_{4} x_{1}^{2}+a_{5} x_{1} x_{2}+a_{6} x_{2}^{2}
\end{array}\right) .
$$

Portinto, $p(x) \in \operatorname{Lrer}^{r}\left(M_{2}^{\mathrm{l}}\right)$, se

$$
\left[\begin{array}{l}
x_{2} \frac{\partial a_{1} x_{1}^{2}+a_{2} x_{1} x_{2}+a_{3} x_{2}^{2}}{\partial x_{1}}-\left(a_{4} x_{1}^{2}+a_{5} x_{1} x_{2}+a_{6} x_{2}^{2}\right) \\
x_{2} \frac{\partial a_{4} x_{1}^{2}+a_{5} x_{1} x_{2}+a_{6} x_{2}^{2}}{\partial x_{1}}
\end{array}\right]=0
$$

('lltion.

$$
\left\{\begin{array}{l}
2 a_{1} x_{1} x_{2}+a_{2} x_{2}^{2}=a_{4} x_{1}^{2}+a_{5} x_{1} x_{2}+a_{6} x_{2}^{2}, \\
2 a_{4} x_{1}+a_{5} x_{2}=0 .
\end{array}\right.
$$

A segunda equação do sistema acima implica que $a_{4}=a_{5}=0$ : e portanto,

$$
\left\{\begin{array}{l}
2 a_{1} x_{1} x_{2}+a_{2} x_{2}^{2}=a_{6} x_{2}^{2} \\
p_{2}(x)=a_{6} x_{2}^{2}
\end{array}\right.
$$

logo, temos da primeira equação que $a_{2}=a_{6}, a_{1}=0$.

Daí,

$$
\left\{\begin{array}{l}
p_{1}(x)-a_{6} x_{1} x_{2}+a_{3} x_{2}^{2} \\
p_{2}(x)=a_{6} x_{2}^{2}
\end{array}\right.
$$


ont sejili

$$
\operatorname{Ker}\left(M_{2}^{1}\right)=\left\{\left(\begin{array}{c}
x_{2}^{2} \\
0
\end{array}\right) \cdot\left(\begin{array}{c}
x_{1} x_{2} \\
x_{2}^{2}
\end{array}\right)\right\}
$$

Assim. escollemos o complementar de $\operatorname{Ker}\left(M_{2}^{1}\right)$, dado por:

$$
\left(\operatorname{Ker}\left(M_{2}^{1}\right)\right)^{c}=\left\{\left(\begin{array}{c}
x_{1}^{2} \\
0
\end{array}\right),\left(\begin{array}{c}
0 \\
x_{1} x_{2}
\end{array}\right),\left(\begin{array}{c}
0 \\
x_{1}^{2}
\end{array}\right) \cdot\left(\begin{array}{c}
x_{1} x_{2} \\
0
\end{array}\right)\right\} .
$$

Usando a expressão em (3.49), conseguimos:

$$
F_{2}(\Phi(x))=2 \alpha x_{1} x_{2},
$$

entian.

$$
f_{2}(x, 0)=\left[\begin{array}{c}
\frac{4 \alpha}{3} \\
4 \alpha \\
2 \alpha(I-\pi) X_{0}
\end{array}\right] x_{1} x_{2}
$$

Como $P_{I, 2}^{2}=I$, pois $\operatorname{Im}\left(M_{2}^{2}\right)=V_{2}^{2}(\operatorname{Ker}(\pi))$, temos que existe uma nuica $h \in V_{2}^{2}\left(Q^{1}\right)$ tal chue:

$$
\left(M_{2}^{2} h\right)(x)=(I-\pi) X_{0} x_{1} x_{2},
$$

(oll seja,

$$
h(x)=\left(M_{2}^{2}\right)^{-1}(I-\pi) X_{0} x_{1} x_{2} .
$$

Considerando a decomposição de $V_{2}^{2}\left(\mathbb{R}^{2}\right)$,

$$
V_{2}^{2}\left(\mathbb{R}^{2}\right)=\operatorname{Im}\left(M_{2}^{1}\right) \oplus\left(\operatorname{Im}\left(M_{2}^{1}\right)\right)^{c},
$$

com $\left(\operatorname{Im}\left(M_{2}^{1}\right)\right)^{r}$, dado em (3.43), obtemos:

$$
f_{2}^{\prime}(x, 0)=\frac{2 \alpha}{3}\left(\begin{array}{c}
2 x_{1} x_{2} \\
0
\end{array}\right)+4 \alpha\left(\begin{array}{c}
0 \\
x_{1} x_{2}
\end{array}\right)
$$

$\operatorname{com} \frac{2 \alpha}{3}\left(\begin{array}{c}2 x_{1} x_{2} \\ 0\end{array}\right) \in \operatorname{Im}\left(M_{2}^{1}\right)$

Desse modo, como $\left(M_{2}^{1}\right)^{-1}\left(\operatorname{Im}\left(M_{2}^{1}\right)\right)=\left(\operatorname{Ker}\left(M_{2}^{1}\right)\right)^{c}$, conscguimos:

$$
U_{2}^{1}(x)=\left(M_{2}^{1}\right)^{-1} P_{I, 2}^{1} f_{2}^{1}(x, 0)=\left(M_{2}^{1}\right)^{-1}\left(\frac{2 \alpha}{3}\left(\begin{array}{c}
2 x_{1} x_{2} \\
0
\end{array}\right)\right)=\frac{2 \alpha}{3}\left(\begin{array}{c}
x_{1}^{2} \\
0
\end{array}\right),
$$

pois.

$$
M_{2}^{1}\left(\left(\begin{array}{c}
x_{1}^{2} \\
0
\end{array}\right)\right)=\left(\begin{array}{c}
2 x_{1} x_{2} \\
0
\end{array}\right)
$$


Concluindo temos que:

$$
U_{2}(x)=2 \alpha\left[\begin{array}{c}
\frac{x_{1}^{2}}{3} \\
0 \\
h(x)
\end{array}\right]
$$

A pratir destre monento, passaremos an cálculo dos termos de ordem trés.

Obtemess da equacion (3.51), cue a parte não linear é dada por:

$$
\begin{aligned}
F(\Phi x+y)= & \alpha(\Phi(0) x+y(0))[(\Phi(0) x+y(0))-(\phi(-1) x+y(-1))]+ \\
& +\beta(\Phi(0) x+y(0))(\phi(-1) x+y(-1))^{2}=\frac{1}{2} F_{2}(x, y)+\frac{1}{3 !} F_{3}(x, y),
\end{aligned}
$$

onde

$$
\begin{aligned}
f_{2}(x, y)= & {\left[2 \times\left(x_{1} x_{2}+x_{1}(y(0)-y(-1))+x_{2} y(0)-y(0) y(-1)+y^{2}(0)\right)\right] . } \\
f_{3}(x, y)= & {\left[3 ! \beta \left(x_{1}\left(x_{1}-x_{2}\right)^{2}+2 x_{1}^{2} y(-1) \cdots 2 x_{1} x_{2} y(-1)+x_{1} y^{2}(-1)+x_{1}^{2} y(0)-\right.\right.} \\
& \left.\left.-2 x_{1} x_{2} y(0)+2 x_{1} y(-1) y(0)+x_{2}^{2} y(0)-2 x_{2} y(-1) y(0)+y^{2}(-1) y(0)\right)\right] .
\end{aligned}
$$

Portanto, a cquação diferencial definida sobre o espaço $P$ é dada por:

$$
\dot{x}=B x+\frac{1}{2}\left[\begin{array}{c}
\frac{2}{3} \\
2
\end{array}\right] F_{2}(x, y)+\frac{1}{3 !}\left[\begin{array}{c}
\frac{2}{3} \\
2
\end{array}\right] F_{3}(x, y) .
$$

Agora, fazendo a mundança de variável

$$
(x, y)-\cdots\left(x+\frac{1}{2} U_{2}^{\mathrm{I}}(x), y+\frac{1}{2} U_{2}^{2}(x)\right)
$$

na equação acima, obleemos,

$$
\begin{aligned}
\left(I+\frac{1}{2} D_{x} U_{2}^{1}(x)\right) x= & B\left(x+\frac{1}{2} U_{2}^{1}(x)\right)+\frac{1}{2} f_{2}^{1}\left(x+\frac{1}{2} U_{2}^{1}(x), y+\frac{1}{2} U_{2}^{2}(x)\right)+ \\
& +\frac{1}{3 !} f_{3}^{1}\left(x+\frac{1}{2} U_{2}^{1}(x), y+\frac{1}{3 !} U_{2}^{2}(x)\right) .
\end{aligned}
$$

Jaí, usando as expressoos en (3.53), conseguimos:

$$
f_{2}^{1}\left(x+\frac{1}{2} U_{2}^{1}(x) \cdot y+\frac{1}{2} U_{2}^{2}(x)\right)=f_{2}^{1}(x, y)+2 \alpha\left[\begin{array}{c}
\frac{2}{3} \\
2
\end{array}\right]\left[\alpha r_{1}(h(x)(0)-h(x)(-1))+\right.
$$




$$
\begin{aligned}
& +\frac{r}{3} r_{1}^{2}(y(0) \cdot y(-1))+\frac{r}{3} x_{1}^{2} x_{2}+2 \alpha y(0) h(x)(0)+x x_{2} h(x)(0)-\alpha y(0) h(x)(-1)- \\
& -\alpha y(-1) h(x)(0)] \\
& f_{3}^{1}\left(x+\frac{1}{2} l_{2}^{1}(x), y+\frac{1}{3 !} U_{2}^{2}(x)\right)=f_{3}^{1}(x, y)+O\left(|(x, y)|^{4}\right)
\end{aligned}
$$

Considerando a inversa do operador $\left(1+\frac{1}{2} D_{x} U_{2}^{1}(x)\right)$ como em (3.17) e as equação (3.54) acima, obtemos:

$$
\begin{aligned}
& \therefore=\left(I-\frac{1}{2} D_{x} U_{2}^{1}(x)+\frac{1}{(2)^{2}} D_{x} U_{2}^{1}(x) D_{x} U_{2}^{1}(x)+O\left(|x|^{4}\right)\right) . \\
& \left\{B x+\frac{1}{2} B U_{2}^{1}(x)+\frac{1}{2}\left[f_{2}^{1}(x, y)+2 \alpha\left[\begin{array}{l}
\frac{2}{3} \\
2
\end{array}\right]\left[\alpha x_{1}(h(x)(0)-h(x)(-1))+\right.\right.\right. \\
& +\frac{\alpha}{3} x_{1}^{2}(y(0)-y(-1))+\frac{\alpha}{3} x_{1}^{2} x_{2}+2 \alpha y(0) h(x)(0)+\alpha x_{2} h(x)(0)-\alpha y(0) h(x)(-1)- \\
& \left.-\alpha y(-1) h(x)(0)]]+\frac{1}{3 !} f_{3}^{1}(x, y)+O\left(|(x, y)|^{4}\right) \quad\right\} \\
& =B x+\frac{1}{2} g_{2}^{1}(x, y)+\frac{1}{3 !}\left[f_{3}^{1}(x, y)+4 \alpha\left[\begin{array}{l}
1 \\
3
\end{array}\right]\left[\alpha x_{1}(h(x)(0)-h(x)(-1))+\right.\right. \\
& +\frac{\alpha}{3} x_{1}^{2}(y(0)-y(-1))+\frac{\alpha}{3} x_{1}^{2} x_{2}+2 \alpha y(0) h(x)(0)+\alpha x_{2} h(x)(0)-\alpha y(0) h(x)(-1)- \\
& \left.-(x y(-1) h(x)(0)]-\frac{3}{4} D_{x} U_{2}^{1}(x)\left[f_{2}^{1}(x, y)-\left(D_{x} U_{2}^{1}(x) B x-B U_{2}^{1}(x)\right)\right]\right]+ \\
& +O\left(|(x, y)|^{4}\right) \\
& =B x+\frac{1}{2} g_{2}^{1}(x, y)+\frac{1}{3 !}\left[f_{3}^{1}(x, y)+4 \alpha\left[\begin{array}{l}
1 \\
3
\end{array}\right]\left[\alpha x_{1}(h(x)(0)-h(x)(-1))+\right.\right. \\
& +\frac{\alpha}{3} x_{1}^{2}(y(0)-y(-1))+\frac{\alpha}{3} x_{1}^{2} x_{2}+2 \alpha y(0) h(x)(0)+\alpha x_{2} h(x)(0)-\alpha y(0) h(x)(-1) \cdots
\end{aligned}
$$




$$
\left.-\alpha y(-1) h(x)(0)]-\frac{3}{4} D_{x} U_{2}^{\prime}(x) g_{2}^{1}(x, y)\right]+O\left(|(x, y)|^{4}\right)
$$

Daí. parar $y=0$, temos (que:

$$
\begin{aligned}
i:= & 33 x+\frac{1}{2} g_{2}^{1}(x, 0)+\frac{1}{3 !}\left[f_{3}^{1}(x, 0)+4 \alpha^{2}\left[\begin{array}{l}
1 \\
3
\end{array}\right]\left[x_{1}(h(x)(0)-h(x)(-1))+\right.\right. \\
& \left.\left.+\frac{1}{3} x_{1}^{2} x_{2}+x_{2} h(x)(0)\right]\right]+O\left(|x|^{4}\right),
\end{aligned}
$$

oll scja

$$
\begin{aligned}
\dot{x}= & B x+\frac{1}{2}\left[\begin{array}{c}
0 \\
2 \alpha x_{1} x_{2}
\end{array}\right]+\frac{1}{3 !}\left[\left[\begin{array}{l}
1 \\
3
\end{array}\right] 4 \beta\left(x_{1}\left(x_{1}-x_{2}\right)^{2}\right)+4 \alpha^{2}\left[+\frac{1}{3} x_{1}^{2} x_{2}+\right.\right. \\
& \left.\left.+x_{1}(h(x)(0)-h(x)(-1))+x_{2} h(x)(0)\right]\right]+O\left(|x|^{4}\right) .
\end{aligned}
$$

Assim, os termos de ordem três que surgem após fazermos a mudança de variável para os termos de ordem dois, são dados por:

$$
\begin{aligned}
f_{3}^{1}(x, 0)= & {\left[\left[\begin{array}{l}
1 \\
3
\end{array}\right] 4 \beta\left(x_{1}\left(x_{1}-x_{2}\right)^{2}\right)+4 \alpha^{2}\left[+\frac{1}{3} x_{1}^{2} x_{2}+x_{1}(h(x)(0)-h(x)(-1))+\right.\right.} \\
& \left.\left.+x_{2} h(x)(0)\right]\right] .
\end{aligned}
$$

Na sequência, para obtermos $g_{3}^{1}(x, 0)$ como em (3.20), precisamos escollıer o espaço complementar de $\operatorname{Im}\left(M_{3}^{3}\right)$.

Consideremos a base canônica de $V_{3}^{2}\left(\mathbb{R}^{2}\right)$,

$$
\left(\begin{array}{c}
x_{1}^{3} \\
0
\end{array}\right),\left(\begin{array}{c}
x_{1}^{2} x_{2} \\
0
\end{array}\right),\left(\begin{array}{c}
x_{1} x_{2}^{2} \\
0
\end{array}\right),\left(\begin{array}{c}
x_{2}^{3} \\
0
\end{array}\right),\left(\begin{array}{c}
0 \\
x_{1}^{3}
\end{array}\right),\left(\begin{array}{c}
0 \\
x_{1}^{2} x_{2}
\end{array}\right),\left(\begin{array}{c}
0 \\
x_{1} x_{2}^{2}
\end{array}\right),\left(\begin{array}{c}
0 \\
x_{2}^{3}
\end{array}\right)
$$

então, suas respectivas imagens, sob o operador

$$
M_{3}^{1}(p(x))=\left[\begin{array}{l}
x_{2} \frac{\partial p_{1}(x)}{\partial x_{1}}-p_{2}(x) \\
x_{2} \frac{\partial p_{2}(x)}{\partial x_{1}}
\end{array}\right]
$$


siis:

$$
\left(\begin{array}{c}
3 r_{1}^{3} x_{2} \\
0
\end{array}\right) \cdot\left(\begin{array}{c}
2 x_{1} x_{2}^{2} \\
0
\end{array}\right) \cdot\left(\begin{array}{c}
x_{2}^{3} \\
0
\end{array}\right) \cdot\left(\begin{array}{l}
0 \\
0
\end{array}\right) \cdot\left(\begin{array}{c}
-x_{1}^{3} \\
3 x_{1}^{2} x_{2}
\end{array}\right) \cdot\left(\begin{array}{c}
-x_{1}^{2} x_{2} \\
2 x_{1} x_{2}^{2}
\end{array}\right) \cdot\left(\begin{array}{c}
-x_{1} \cdot x_{2}^{2} \\
x_{2}^{3}
\end{array}\right) \cdot\left(\begin{array}{c}
-x_{2}^{3} \\
0
\end{array}\right) .
$$

Portante temos gune:

$$
\begin{aligned}
\operatorname{lm}\left(M_{3}^{1}\right)=\operatorname{ger} & \left\{\left(\begin{array}{c}
3 x_{1}^{2} x_{2} \\
0
\end{array}\right),\left(\begin{array}{c}
2 x_{1} x_{2}^{2} \\
0
\end{array}\right),\left(\begin{array}{c}
x_{2}^{3} \\
0
\end{array}\right),\right. \\
& \left.\left(\begin{array}{c}
-x_{1}^{3} \\
3 x_{1}^{2} x_{2}
\end{array}\right),\left(\begin{array}{c}
-x_{1}^{2} x_{2} \\
2 x_{1} x_{2}^{2}
\end{array}\right),\left(\begin{array}{c}
-x_{1} x_{2}^{2} \\
x_{2}^{3}
\end{array}\right)\right\} .
\end{aligned}
$$

Com isso, porlemos tomar o espaço complementar de $\operatorname{Im}\left(M_{3}^{1}\right)$,

$$
\operatorname{Im}\left(M_{3}^{1}\right)^{r}=\operatorname{ger}\left\{\left(\begin{array}{c}
0 \\
x_{1}^{3}
\end{array}\right),\left(\begin{array}{c}
0 \\
x_{1}^{2} x_{2}
\end{array}\right)\right\} .
$$

Como o polinômio homogêneo $h$ pertence à $V_{2}^{2}\left(Q^{1}\right)$, podemos escrever:

$$
h(x)(\theta)=h_{(2,0)}(\theta) x_{1}^{2}+h_{(1,1)}(\theta) x_{1} x_{2}+h_{(0,2)}(\theta) x_{2}^{2} .
$$

(om) h(i,j) $\subseteq Q^{1}, i, j=1,20 i+j=2$.

Substituindo $(3.55)$ em $f_{3}^{1}(x, 0)$ e tomando sua decomposição em relaçáo a decomposicaio

$$
V_{2}^{2}\left(\mathbb{R}^{2}\right)=\operatorname{Im}\left(M_{3}^{1}\right) \oplus\left(\operatorname{Im}\left(M_{3}^{1}\right)\right)^{c} .
$$

obtemos:

$$
\begin{aligned}
& f_{3}^{1}(x, 0)=\left[\left(-\frac{8}{3} \beta+\frac{1 \alpha^{2}}{9}+\frac{4 \alpha^{2}}{3} h_{(1.1)}(0)-\frac{4 \alpha^{2}}{3} h_{(1.1)}(-1)+\frac{4 \alpha^{2}}{3} h_{(2,0)}(0)\right) .\right. \\
& \left(\begin{array}{c}
3 x_{1}^{2} x_{2} \\
0
\end{array}\right)+\left(2 \beta+2 \alpha^{2} h_{(0,2)}(0)-2 \alpha^{2} h_{(1,1)}(-1)+2 \alpha^{2} h_{(1,1)}(0)\right) \text {. } \\
& \left(\begin{array}{c}
2 x_{1} x_{2}^{2} \\
0
\end{array}\right)+\left(4 \alpha^{2} h_{(0,2)}(0)\right) \cdot\left(\begin{array}{c}
x_{2}^{3} \\
0
\end{array}\right)+\left(12 \alpha^{2} h_{(1,1)}(0)\right)\left(\begin{array}{c}
0 \\
x_{2}^{3}
\end{array}\right)+ \\
& +\left(-4 \beta+4 \alpha^{2} h_{(2,0)}(0)+4 \alpha^{2} h_{(1,1)}(-1)\right) \cdot\left(\begin{array}{c}
-x_{1}^{3} \\
3 x_{1}^{2} x_{2}
\end{array}\right)+ \\
& \left.\left(12 \beta+4 \alpha^{2} h_{(0,2)}(0)-4 \alpha^{2} h_{(0,2)}(-1)+4 \alpha^{2} h_{(1,1)}(0)\right) \cdot\left(\begin{array}{c}
0 \\
x_{1} x_{2}^{2}
\end{array}\right)\right]+ \\
& +\left[12 \beta\left(\begin{array}{c}
0 \\
\left(x_{1}^{3}-x_{1}^{2} x_{2}\right)
\end{array}\right)+12 \alpha^{2}\left(\begin{array}{c}
0 \\
\left(h_{(2,0)}(0)-h_{(2,0)}(-1)\right) x_{1}^{3}
\end{array}\right)+\right.
\end{aligned}
$$

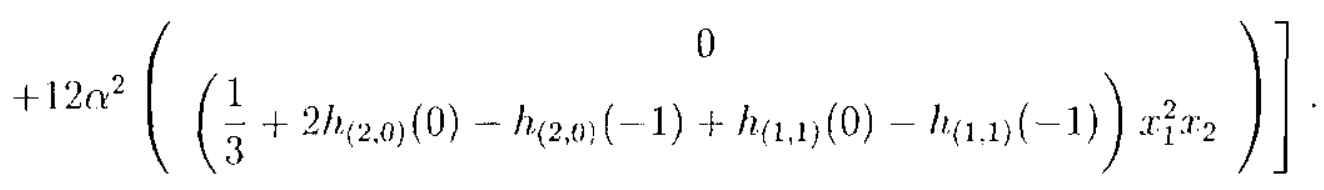


Assim. comseguinos de (3.20), (pue os temos de ordem tres da forma normal sobue a varindate central sius dados por:

$$
\begin{aligned}
g_{3}^{\prime}(x, 0)= & \left(I-P_{3,3}^{1}\right) \tilde{f}_{3}^{1}(x, 0)=12 \beta\left(\left(x_{1}^{3}-x_{1}^{2} x_{2}\right)\right)+ \\
& +12 \alpha^{2}\left(\left(h_{(2,0)}(0)-h_{(2,0)}(-1)\right) x_{1}^{3}\right)+ \\
& +12 \alpha^{2}\left(\left(\frac{1}{3}+2 h_{(2,0)}(0)-h_{(2,0)}(-1)+h_{(1,1)}(0)-h_{(1,1)}(-1)\right) x_{1}^{2} x_{2}\right)
\end{aligned}
$$

Para cue possamos explicitar os termos de ordem três da forma normal sobre a variedade central em termos dos cocficientes da equação original (3.51), é preciso calcularmos $h_{(2,0)}$ e $h_{(1,1)}$ pela resolução de (3.52). Como essa equação é considerada em un espaço de dimensào infinita, temos que essa é lma equação funcional, na forma de um problena de valor de contono para una equaçäo diferencial ordinária em $\mathbb{R}^{3}$.

Do fato:

Usando a definição de $M_{2}^{2}$, conseguinos que:

$$
D_{x} h(x) B x-A_{Q^{1}} h(x)=(I-\pi) X_{0} x_{1} x_{2}
$$

Uma ve que $h_{(i, j)} \in Q^{\mathrm{l}}, i, j=1,2 \mathrm{e} i+j=2$, temos que

$$
A_{Q^{1}} h_{(i, j)}=A h_{(i, j)}
$$

e portanto.

$$
x_{2} \frac{\partial h(x)(\theta)}{\partial x_{1}}-\dot{h}(x)(\theta)+X_{0}\left[\dot{h}_{(x)}(0)-L(h(x))\right]=\left[X_{0}-\left(\frac{1}{3}+2 \theta\right)\right] x_{1} x_{2}
$$

onde ir denotia a derivada de $h(x)(\theta)$ com relação à $\theta$.

Substituindo a expressão de $h$, como $\mathrm{cm}(3.55)$, obtemos

$$
\begin{aligned}
& \left(-\dot{h}_{(2,0)}(\theta)\right) x_{1}^{2}+\left(2 h_{(\theta)}(\theta, 0)-\dot{h}_{(1,1)}(\theta)\right) x_{1} x_{2}+\left(h_{(1,1)}(\theta)-\dot{h}_{(0,2)}(\theta)\right) x_{2}^{2}+ \\
& X_{0}\left[\left(\dot{h}_{(2,0)}(0)-h_{(2,0)}(0)+h_{(2,0)}(-1)\right) x_{1}^{2}+\left(\dot{h}_{(1,1)}(0)-h_{(1,1)}(0)+h_{(1,1)}(-1)\right) x_{1} x_{2}+\right. \\
& \left.\left(h_{(0,2)}(0)-h_{(0,2)}(0)+h_{(0,2)}(-1)\right) x_{2}^{2}\right]=-\left(\frac{2}{3}+2 \theta\right) x_{1} x_{2}+X_{0} x_{1} x_{2},
\end{aligned}
$$




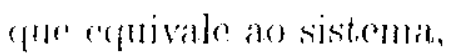

$$
\begin{aligned}
\dot{h}_{(2,0)}(\theta) & =0 \\
2 h_{(2,0)}(\theta)-\dot{h}_{(1,1)}(\theta) & =-\left(\frac{2}{3}+2 \theta\right), \\
h_{(1,1)}(\theta)-\dot{h}_{(0,2)}(\theta) & =0
\end{aligned}
$$

conn as condiçoes de contono

$$
\begin{aligned}
& \left(h_{(2,0)}(0)-h_{(2,0)}(0)+h_{(2,0)}(-1)\right)=0, \\
& \left(h_{h(1,1)}(0)-h_{(1,1)}(0)+h_{\langle 1,1)}(-1)\right)=1, \\
& \left(\dot{h}_{(0,2)}(0)-h_{\{0,2)}(0)+h_{\{0,2)}(-1)\right)=0 .
\end{aligned}
$$

Obtemos da primeira equação de $(3.57)$ que $h_{(2,0)}$ é constante, mas as funções constantes pertencem à $P$, além disso, $h_{(2,0)} \in Q^{1}$, portanto $h_{(2,0)} \equiv 0$.

Assinu, resolvendo (3.57) e (3.58) para obtermos $h_{(1,1)}$, temos que:

$$
h_{(1,1)}(\theta)=\theta^{2}+\frac{2}{3} \theta+c
$$

(r) $111 \in \mathbb{R}$.

Para o cáleulo de $g_{3}^{l}(x, 0)$, nào é necessário o cálculo de $C$, embora poderiamos ter feito usando o fato que $h_{(1,1)} \in Q^{1}=\left\{\phi \in C^{1}:(\Psi . \phi)=0\right\}$.

Substituindo os valores obtidos acima em (3.56), conseguimos:

$$
g_{3}^{3}(x, 0)=12 \beta\left[\begin{array}{c}
0 \\
x_{1}^{3}-x_{1}^{2} x_{2}
\end{array}\right] .
$$

Portanto, o fluxo sobre a variedade central para a equação (3.51), é dado na forma normal até terecira ordem por:

$$
\left\{\begin{array}{l}
\dot{x}_{1}=x_{2}+O\left(|x|^{4}\right) \\
\dot{x}_{2}=2 \alpha x_{1} x_{2}+2 \beta x_{1}^{3}-2 \beta x_{1}^{2} x_{2}+O\left(|x|^{4}\right) .
\end{array}\right.
$$

\section{Observação:}

A principal diferença que podemos observar entre o cálculo da forma normal relativamente a $u$ m espaço invariante m-dimensional para equaçóes diferenciais funcionais retardadas e ordinárias, consiste na necessidade de considerarmos umal equação diferenrial funcional (no exemplo um problema de valor de contorno para umna equação diferencial ordinária cun $\mathbb{R}^{3}$ ) ao invés de unı equação algébrica. Esse fato foi ilustrado nessa última aplicaçăo. 


\subsection{Singularidade do tipo Bogdanov-Takens com um parâmetro}

Vesta seçü. encerraremos o capítulo calculando a forma nomal para ecpuaçoes dilerenriais funcionais retardadas com un parânetro e singularidade do tipo Bogdanov-Takens.

Comsiderenos a equaça diferencial funcional retardada com um paranetro.

$$
\dot{z}(l)=L(\alpha)\left(z_{t}\right)+F\left(z_{t}, \alpha\right)
$$

onde zi pertence à $C=C\left([-r, 0], \mathbb{R}^{\prime \prime}\right)$, o paranetro a pertence à $V$, una vizinhança do zero en $\mathbb{R}^{n}, L: V \rightarrow \mathfrak{L}\left(C, \mathbb{R}^{n}\right)$ é de classe $C^{1}$ e $F: C \times V+\mathbb{R}^{n}$ é uma função de classe $C^{N}, N \geq 2, \operatorname{com} F(0,0)=0$ e $D F(0,0)=0$.

Como na Seção 3.5, consideraremos o parânetro $\alpha$ como una nova variável, intro duzindo a equação $\dot{\alpha}=0$. Mas, como a equação para $\alpha$ está na forma mais simples possível, basta considerarmos a equação (3.59) nas variáveis $z_{t} \in C$ e $\alpha \in V$.

Definindo $L_{0}=L(0)$, segue que,

$$
L(\alpha)\left(z_{t}\right)-L_{0}\left(z_{t}\right)=O\left(\left|\left(z_{t}, \alpha\right)\right|^{2}\right),
$$

e portanto, podemos reescrever a equação $(3.59)$

$$
\dot{z}(t)=L_{0}\left(z_{t}\right)+\left[F\left(z_{t}, \alpha\right)+L(\alpha)\left(z_{t}\right)-L_{0}\left(z_{t}\right)\right],
$$

omle $\left[F\left(z_{t}, \alpha\right)+L(\alpha)\left(z_{t}\right)-L_{0}\left(z_{t}\right)\right]$ é a parte não linear nas variáveis $z_{t}$ e $\alpha$, e entào, temos que a equação linearizadla associada à equaçâo (3.59), é dada por:

$$
\dot{z}(t)=L_{0}\left(z_{t}\right) \text {. }
$$

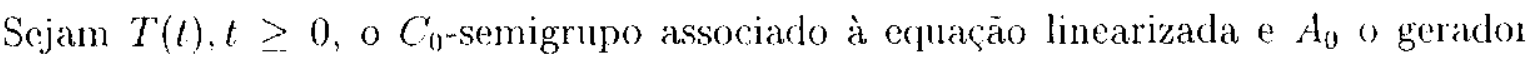
infinitesimal associado a esse $C_{0}$-semigrupo. Tomemos $\Lambda$ um subconjunto finito o nãovazio do autovalores do gerador infinitesimal $A_{0}$ e suponhamos que $\Lambda$ contém $m$ elementos onde cada elemento é contado tantas vezes quanto sua multiplicidade cono raiz de equação caraclerística $(3.3)$.

Tomemos as expansōes de Taylor para $L$ e $F$,

$$
\begin{aligned}
L(\alpha) & =L_{0}+\sum_{j=2}^{r-1} L_{j}(\alpha)+O\left(|\alpha|^{r-1}\right), \\
F\left(z_{t}, \alpha\right) & =\sum_{j=2}^{r-1} \frac{1}{j !} F_{j}\left(z_{t}, \alpha\right)+O\left(\left|\left(z_{t}, \alpha\right)\right|^{r}\right),
\end{aligned}
$$

onde $L_{j}(\alpha)$ ć de ordem $j-1$ em $\alpha$ e $F_{j}\left(z_{t}, \alpha\right)$ é de ordem $j$ en $\left(z_{t}, \alpha\right)$. Observemos que $L_{j}(\alpha) z_{t}$ é de orden $j$ em $\left(z_{t}, \alpha\right)$.

Suponhamos que a equação caractcrística (3.3):

(i) tenla $\lambda=0$ como autovalor com ascendente dois;

(ii) não tenha nenhum autovalor, além de $\lambda=0$, com parte real zero.

Observemos que $\lambda=0$ ter ascendente dois significa que:

$$
\operatorname{dim}\left(\operatorname{Ker}\left(A_{0}\right)\right)=1, \operatorname{dim}\left(\operatorname{Ker}\left(A_{0}\right)^{2}\right)=2, \operatorname{dim}\left(\operatorname{Ker}\left(A_{0}\right)^{3}\right)=2,
$$


"I' $=\operatorname{Ker}\left(A_{0}\right)^{2}=\mathcal{M}_{0}\left(A_{0}\right)$, onde $\mathcal{M}_{0}\left(A_{0}\right)$ ó o espaço generalizado de $A_{0}$ associado com o comjunto $\Lambda=\{0\}$. Em particular, isso implica que $\lambda=0$ é autovalor de multiplicidade dois.

Visse caso, temos que $P$ e $P^{T}$ têm dimensão dois. Escolhemos as bases $\Phi$ e $\Psi$ de $I^{\prime}$ e $P^{7}$. resperetivamente.

$$
\begin{array}{ll}
\Phi(\theta)=\left(\phi_{1}(\theta), \phi_{2}(\theta)\right), & -1 \leq \theta \leq 0, \\
\Psi(s)=\operatorname{col}\left[\psi_{1}(s), \psi \eta_{2}(s)\right], & 0 \leq s \leq 1,
\end{array}
$$

rom

$$
\phi_{1}(\theta)=\left(\begin{array}{c}
a_{1} \\
a_{2} \\
\vdots \\
a_{n}
\end{array}\right), \phi_{2}(\theta)=\left(\begin{array}{c}
b_{1} \\
b_{2} \\
\vdots \\
b_{n}
\end{array}\right)+\theta\left(\begin{array}{c}
a_{1} \\
a_{2} \\
\vdots \\
a_{n}
\end{array}\right),
$$

$\psi_{1}(s)=\left(\begin{array}{llll}z_{1} & z_{2} & \ldots & z_{n}\end{array}\right) \cdot \psi_{2}(s)=\left(\begin{array}{llll}w_{1} & w_{2} & \ldots & w_{n}\end{array}\right)-s\left(\begin{array}{llll}z_{1} & z_{2} & \ldots & z_{n}\end{array}\right)$,

onde:

$$
\begin{gathered}
\left(\begin{array}{c}
a_{2} \\
a_{2} \\
\vdots \\
a_{n}
\end{array}\right),\left(\begin{array}{c}
b_{1} \\
b_{2} \\
\vdots \\
b_{n}
\end{array}\right) \in \mathbb{R}^{n}, \\
\left(\begin{array}{cccc}
z_{1} & z_{2} & \ldots & z_{n}
\end{array}\right),\left(\begin{array}{llll}
w_{1} & w_{2} & \ldots & w_{n}
\end{array}\right) \in \mathbb{R}^{n^{*}}
\end{gathered}
$$

e assumiremos que $(\Psi, \Phi)=I$.

Então, obtemos que a matriz $B$ tal que as bases $\Phi$ e $\Psi$, satisfazem:

$$
\Lambda_{0} \Phi=\Phi B \text { e } A_{0}^{T} \Psi=B \Psi
$$

$\therefore$ lata por:

$$
B=\left[\begin{array}{ll}
0 & 1 \\
0 & 0
\end{array}\right]
$$

Portanto, para $\alpha=0$, a equação diferencial ordinária bidimensional sobre a variedade central tangente ao espaço $P$ no zero, tem singularidade do tipo Bogdanov-Takens. Determinarmos sua dinâmica, en uma vizinhança de $z_{t}=0 \mathrm{c} \alpha=0$, até os termos de segunda ordem. Da suposição $(i i)$, obtemos que as condições de não-ressonância relativa a $\Lambda=\{(0\}$, da Definição 3.4.1, são satisfeitas, e portanto, do Teorema 3.6.1, temos que a forma normal até segunda ordem para a equaçào diferencial funcional retardada com um parâmetro (3.59), sobre a variedade central, é dada por:

$$
\dot{x}=B x+\frac{1}{2 !} g_{2}^{1}(x, 0, \alpha)+O\left(|(x, \alpha)|^{3}\right)
$$

ondes $g_{2}^{1}(x, 0, \alpha)=\left(I-P_{I m\left(M_{2}^{1}\right)}^{1}\right) \Psi(0)\left[2 L_{2}(\alpha)(\Phi x)+F_{2}(\Phi x, \alpha)\right]$. 
Para a matriz $B$ acima, o operador $M_{2}^{1}$ ó definido por:

$$
\begin{aligned}
& M_{2}^{1}: V_{2}^{\prime}\left(\mathbb{R}^{2}\right) \rightarrow V_{2}^{4}\left(\mathbb{R}^{2}\right), \\
&\left(M_{2}^{1} p\right)(x, r)=\left(\begin{array}{l}
x_{2} \frac{\partial p_{1}(x)}{\partial x_{1}}-p_{2}(x) \\
x_{2} \frac{\partial p_{2}(x)}{\partial x_{1}}
\end{array}\right),
\end{aligned}
$$

com $p=\left(\begin{array}{l}p_{1} \\ p_{2}\end{array}\right)$ e domínio $D\left(M_{2}^{1}\right)=V_{2}^{4}\left(\mathbb{R}^{2}\right)$.

Considerenos a base canonica de $V_{2}^{4}\left(\mathbb{R}^{2}\right)$,

$$
\begin{aligned}
& \left(\begin{array}{c}
x_{1}^{2} \\
0
\end{array}\right),\left(\begin{array}{c}
x_{1} x_{2} \\
0
\end{array}\right),\left(\begin{array}{c}
x_{2}^{2} \\
0
\end{array}\right),\left(\begin{array}{c}
x_{1} \alpha_{i} \\
0
\end{array}\right),\left(\begin{array}{c}
x_{2} \alpha_{i} \\
0
\end{array}\right),\left(\begin{array}{c}
\alpha_{1}^{2} \\
0
\end{array}\right), \\
& \left(\begin{array}{c}
\alpha_{1} \alpha_{2} \\
0
\end{array}\right),\left(\begin{array}{c}
\alpha_{2}^{2} \\
0
\end{array}\right),\left(\begin{array}{c}
0 \\
x_{1}^{2}
\end{array}\right),\left(\begin{array}{c}
0 \\
x_{1} x_{2}
\end{array}\right),\left(\begin{array}{c}
0 \\
x_{2}^{2}
\end{array}\right),\left(\begin{array}{c}
0 \\
x_{1} \alpha_{i}
\end{array}\right), \\
& \left(\begin{array}{c}
0 \\
x_{2} \alpha_{i}
\end{array}\right),\left(\begin{array}{c}
0 \\
\alpha_{1}^{2}
\end{array}\right),\left(\begin{array}{c}
0 \\
\alpha_{1} \alpha_{2}
\end{array}\right),\left(\begin{array}{c}
0 \\
\alpha_{2}^{2}
\end{array}\right), \quad(i=1,2),
\end{aligned}
$$

(ntãu, suas imagens sol, o operador $M_{2}^{1}$ são, respectivamente,

$$
\begin{aligned}
& \left(\begin{array}{c}
2 x_{1} x_{2} \\
0
\end{array}\right),\left(\begin{array}{c}
x_{2}^{2} \\
0
\end{array}\right),\left(\begin{array}{l}
0 \\
0
\end{array}\right),\left(\begin{array}{c}
x_{2} \alpha_{i} \\
0
\end{array}\right),\left(\begin{array}{l}
0 \\
0
\end{array}\right),\left(\begin{array}{l}
0 \\
0
\end{array}\right) \cdot\left(\begin{array}{l}
0 \\
0
\end{array}\right) \cdot\left(\begin{array}{l}
0 \\
0
\end{array}\right) . \\
& \left(\begin{array}{c}
-x_{1}^{2} \\
2 x_{1} x_{2}
\end{array}\right),\left(\begin{array}{c}
-x_{1} x_{2} \\
x_{2}^{2}
\end{array}\right),\left(\begin{array}{c}
-x_{2}^{2} \\
0
\end{array}\right),\left(\begin{array}{c}
-x_{1} \alpha_{i} \\
x_{2} \alpha_{i}
\end{array}\right),\left(\begin{array}{c}
-x_{2} \alpha_{i} \\
0
\end{array}\right) \cdot\left(\begin{array}{c}
-\alpha_{1}^{2} \\
0
\end{array}\right), \\
& \left(\begin{array}{c}
-x_{1} \alpha_{2} \\
0
\end{array}\right),\left(\begin{array}{c}
-\alpha_{2}^{2} \\
0
\end{array}\right), \quad(i=1,2) .
\end{aligned}
$$

Assim, podemos tomar a decomposição $V_{2}^{4}\left(\mathbb{R}^{2}\right)=\operatorname{Im}\left(M_{2}^{1}\right) \oplus \operatorname{Im}\left(M_{2}^{1}\right)^{c}$, com o espaço complementar $\operatorname{Im}\left(M_{2}^{1}\right)^{c}$ gerado pelos clementos:

$$
\begin{aligned}
& \left(\begin{array}{c}
0 \\
x_{1}^{2}
\end{array}\right) \cdot\left(\begin{array}{c}
0 \\
x_{1} x_{2}
\end{array}\right),\left(\begin{array}{c}
0 \\
x_{1} \alpha_{1}
\end{array}\right),\left(\begin{array}{c}
0 \\
x_{1} \alpha_{2}
\end{array}\right), \\
& \left(\begin{array}{c}
0 \\
x_{2} \alpha_{1}
\end{array}\right),\left(\begin{array}{c}
0 \\
x_{2} \alpha_{2}
\end{array}\right),\left(\begin{array}{c}
0 \\
\alpha_{1}^{2}
\end{array}\right),\left(\begin{array}{c}
0 \\
\alpha_{1} \alpha_{2}
\end{array}\right),\left(\begin{array}{c}
0 \\
\alpha_{2}^{2}
\end{array}\right) .
\end{aligned}
$$

Podemos escrever $f_{2}^{1}(x, 0, \alpha)=\Psi(0)\left[2 L_{2}(\alpha)(\Phi x)+F_{2}^{1}(\Phi x, \alpha)\right]$, da forma: $f_{2}^{1}(x, 0, \alpha)=\left(\begin{array}{l}b_{1} x_{1} \alpha_{1}+b_{2} x_{1} \alpha_{2}+b_{3} x_{2} \alpha_{1}+b_{4} x_{2} \alpha_{2}+A_{(2,0)}^{1} x_{1}^{2}+A_{(1,1)}^{1} x_{1} x_{2}+A_{(0,2)}^{1} x_{2}^{2} \\ c_{1} x_{1} \alpha_{1}+c_{2} x_{1} \alpha_{2}+c_{3} x_{2} \alpha_{1}+c_{4} x_{2} \alpha_{2}+A_{(2,0)}^{2} x_{1}^{2}+A_{(1,1)}^{2} x_{1} x_{2}+A_{(0,2)}^{2} x_{2}^{2}\end{array}\right)$, onde $b_{1}, \ldots, b_{4}, c_{1}, \ldots, c_{4}, A_{(i, j)}^{k}$ são números reais com $i, j, k=1,2$ e $i+j=2$. 
Daí, deromponos $f_{2}^{1}(x, 0, a)$ en relação a decomposiçào acima da seguinte forma:

$$
\begin{aligned}
& f_{2}^{1}(x, 0 . n)-\left[b_{1}\left(\begin{array}{c}
x_{1} \alpha_{1} \\
-x_{2} \alpha_{1}
\end{array}\right)+b_{2}\left(\begin{array}{c}
x_{1} \alpha_{2} \\
-x_{2} \alpha_{2}
\end{array}\right)+b_{3}\left(\begin{array}{c}
x_{2} \alpha_{1} \\
0
\end{array}\right)-b_{4}\left(\begin{array}{c}
x_{2} x_{2} \\
0
\end{array}\right)+\right. \\
& \left.+A_{\{2,0)}^{\prime}\left(\begin{array}{c}
x_{1}^{2} \\
-2 x_{1} x_{2}
\end{array}\right)+A_{(1,1)}^{1}\left(\begin{array}{c}
x_{1} x_{2} \\
0
\end{array}\right)+A_{(0,2)}^{1}\left(\begin{array}{c}
x_{2}^{2} \\
0
\end{array}\right)+A_{(0,2)}^{2}\left(\begin{array}{c}
0 \\
x_{2}^{2}
\end{array}\right)\right] \\
& +\left[c_{1}\left(\begin{array}{c}
0 \\
x_{1} \alpha_{1}
\end{array}\right)+c_{2}\left(\begin{array}{c}
0 \\
x_{1} \alpha_{2}
\end{array}\right)+c_{3}\left(\begin{array}{c}
0 \\
x_{2} \alpha_{1}
\end{array}\right)+c_{4}\left(\begin{array}{c}
0 \\
x_{2} \alpha_{2}
\end{array}\right)+b_{1}\left(\begin{array}{c}
0 \\
x_{2} \alpha_{1}
\end{array}\right)+\right. \\
& \left.+b_{2}\left(\begin{array}{c}
0 \\
x_{2} \alpha_{2}
\end{array}\right)+A_{(2,0)}^{2}\left(\begin{array}{c}
0 \\
x_{1}^{2}
\end{array}\right)+2 A^{1}(2,0)\left(\begin{array}{c}
0 \\
x_{1} x_{2}
\end{array}\right)+A_{(1,1)}^{2}\left(\begin{array}{c}
0 \\
x_{1} x_{2}
\end{array}\right)\right] \text {. }
\end{aligned}
$$

Portanto.

$$
\begin{aligned}
g_{2}^{1}(x, 0, \alpha)= & {\left[c_{1}\left(\begin{array}{c}
0 \\
x_{1} \alpha_{1}
\end{array}\right)+c_{2}\left(\begin{array}{c}
0 \\
x_{1} \alpha_{2}
\end{array}\right)+c_{3}\left(\begin{array}{c}
0 \\
x_{2} \alpha_{1}
\end{array}\right)+c_{1}\left(\begin{array}{c}
0 \\
x_{2} \alpha_{2}
\end{array}\right)+b_{1}\left(\begin{array}{c}
0 \\
x_{2} \alpha_{1}
\end{array}\right)\right.} \\
& \left.+b_{2}\left(\begin{array}{c}
0 \\
x_{2}\left(x_{2}\right.
\end{array}\right)+A_{(2,0)}^{2}\left(\begin{array}{c}
0 \\
x_{1}^{2}
\end{array}\right)+2 A^{1}(2,0)\left(\begin{array}{c}
0 \\
x_{1} x_{2}
\end{array}\right)+A_{(1,1)}^{2}\left(\begin{array}{c}
0 \\
x_{1} x_{2}
\end{array}\right)\right] \\
= & \left(\begin{array}{c}
0 \\
\lambda_{1} x_{1}+\lambda_{2} x_{2}
\end{array}\right)+\left(\begin{array}{c}
0 \\
B_{1} x_{1}^{2}+B_{2} x_{1} x_{2}
\end{array}\right),
\end{aligned}
$$

ondr

$$
B_{1}=A_{(2,0)}^{2}, B_{2}=2 A_{(2,0)}^{1}+A_{(1,1)}^{2},
$$

Cos parânotros de bifurcação são dados por:

$$
\lambda_{1}=\left(c_{1} \alpha_{1}+c_{2} \alpha_{2}\right), \lambda_{2}=\left[\left(b_{1}+c_{3}\right) \alpha_{1}+\left(b_{2}+c_{4}\right) \alpha_{2}\right] .
$$

Portanto, a equação diferencial ordinária para of fluxo da equação (3.59) sobre a varindade central é dada na forma normal até termos de segunda ordem por:

$$
\left\{\begin{array}{l}
\dot{x}_{1}=x_{2}+O\left((|\lambda|+|x|)|x|^{2}\right) \\
\dot{x}_{2}=\lambda_{1} x_{1}+\lambda_{2} x_{2}+a x_{1}^{2}+b x_{1} x_{2}+O\left((|\lambda|+|x|)|x|^{2}\right),
\end{array}\right.
$$

com coeficientes $B_{1}, B_{2}$ e parâmetros $\lambda_{1}, \lambda_{2}$ definidos em (3.62) e (3.63). 


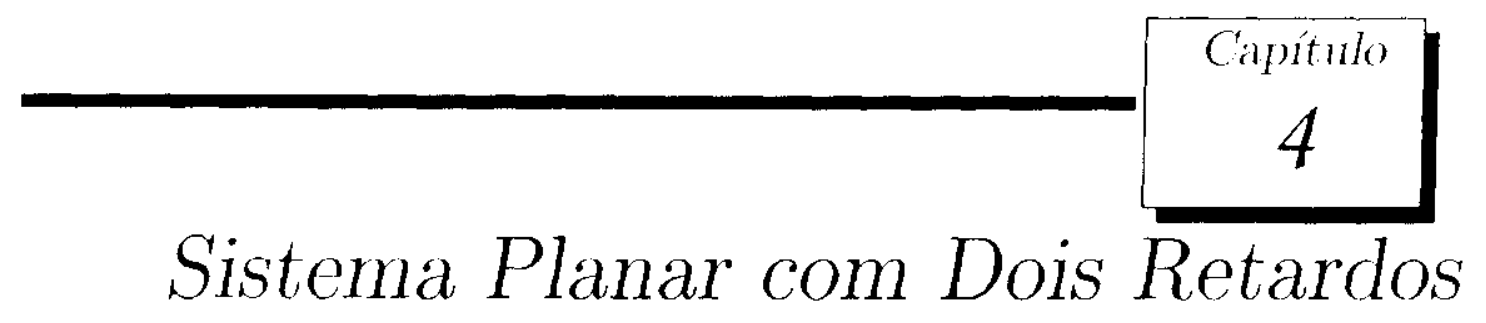

\subsection{Introdução}

Consideremos o sistema planar:

$$
\left\{\begin{array}{l}
\dot{x}_{1}(t)=f_{1}\left(x_{1}(t), x_{2}\left(t-\tau_{2}\right)\right) \\
\dot{x}_{2}(t)=f_{2}\left(x_{1}\left(t-\tau_{1}\right), x_{2}(t)\right),
\end{array}\right.
$$

onde os retardos $\tau_{1}, \tau_{2}$ são não-negativos com $\tau_{1}+\tau_{2}>00 f_{1}, f_{2}: \mathbb{R}^{2} \rightarrow \mathbb{R}$ são de classe $C^{k} . k \geq 2$. $O$ sistema (4.1) inclui $u m$ significativo número de nodelos en dinamicas de populaçós, como por exemplo, o sistema presa-predador.

Nosso objetivo neste capítulo, é determinar as condiçoes para as quais ocorrem as singularidates de Hopf e Bogdanov-Takens cm um ponto de equilíbrio de (4.1). Existe lum significativo número de artigos que analisam a bifurcaça de Hopf para equaçós sinulares a(4.1). Xiac e Ruan (ver [16]) estudaram um caso particular de (4.1), com somente um retardo, onde é analisado a bifurcação de Bogdanov-Takens; Hale e Tanaka ( ver [8]) estudaram equaçoes diferenciais funcionais com dois retardos.

Este capítulo está organizado do seguinte modo. Na Seção 4.2, desenvolveremos o problema de autovalor para a equação linearizada de (4.1) ao redor de $11 n$ ponto de repulibrio $E^{*}$. Na Seçâo 4.3, descreveremos a existência da singularidade de BogdanovTakens com condições sobre o parânetro $\tau=\tau_{1}+\tau_{2}$ e as derivadas parciais de $f_{1}, f_{2}$ no ponto de equilíbrio c calcularemos a forma normal para o modelo presa-predador com dois retardos.

\subsection{O Problema de Autovalor}

Nesta seção, obteremos as condições com relação ao parâmetro $\tau$ e as derivarlas parciais de $f_{1}, f_{2}$ no ponto de equilíbrio $E^{*}$ sob as quais, o ponto de equilíbrio $E^{*} i^{i}$ uma singularialade do tipo Hopf on do tipo Bogdanov-Takens.

Consideremos o sistema planar (4.1), onde $f_{1}, f_{2}$ são funcóes de classe $C^{k}, k \geq 2$, definirlas sobre um conjunto aberto de $D \subset \mathbb{R}^{2}$, os retardos $\tau_{1}$, $\tau_{2}$ são nào-negativos e $\tau=\tau_{1}+\tau_{2}>0$. Assinniremos que $E^{*}=\left(x_{1}^{*}, x_{2}^{*}\right)^{T}$ é um ponto de equilíbrio de (4.1), ou sejar,

$$
f_{1}\left(x_{1}^{*}, x_{2}^{*}\right)=f_{2}\left(x_{1}^{*}, x_{2}^{*}\right)=0 .
$$




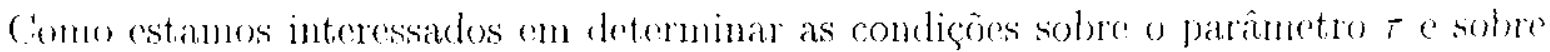
as devivalas parciais de $f_{1}, f_{2} \mathrm{em} E^{*}$ para as quais ocome a singularidade de Hope on Bogdanov-Takens; introduziremos $\tau$ como mom parametro do bifureaçäo.

bazendo a murlancia de variável

$$
u=\frac{t}{\tau}
$$

u10 sistrmal (4.1), com $y_{j}(u)=x_{j}(\tau u), j=1,2$, conseguinos,

$$
\left\{\begin{array}{l}
\dot{y}_{1}(u)=\tau f_{1}\left(y_{1}(u), y_{2}\left(u-r_{2}\right)\right) \\
\dot{y}_{2}(u)=\tau f_{2}\left(y_{1}\left(u-r_{1}\right), y_{2}(u)\right),
\end{array}\right.
$$

com retardos $r_{i}=\frac{\tau_{i}}{\tau} \geq 0, i=1,2, r_{1}+r_{2}=1$.

Considerando o espaço do fase $C=C\left([-1,0], \mathbb{R}^{2}\right)$, a ecunação linearizada para (4.2) enn mana vizinhança $V$ do ponto de equilíbio $E^{*}$ é dada por:

$$
\dot{y}(u)=L_{\tau}\left(y_{u}\right),
$$

roml $y=\left(\begin{array}{l}y_{1} \\ y_{2}\end{array}\right)$ e o operador $L_{\tau}: C \longrightarrow \mathbb{R}^{2}$ é definido por:

$$
L_{\tau}(\phi)=\left(\begin{array}{c}
\tau\left[a_{11} \phi_{1}(0)+a_{12} \phi_{2}\left(-r_{2}\right)\right] \\
\tau\left[a_{21} \phi_{1}\left(-r_{1}\right)+a_{22} \phi_{2}(0)\right]
\end{array}\right),
$$

onde

$$
a_{i j}=\frac{\partial f_{i}\left(E^{*}\right)}{\partial x_{j}}, i, j=1,2 .
$$

Como a equaçĩo característica é definida por:

$$
\Delta(\lambda, \tau):=\operatorname{det}\left(\lambda I-L_{\tau}\left(e^{\lambda \theta}\right)\right)=0
$$

obtemos,

$$
\Delta(\lambda, \tau)=\lambda^{2} \cdots\left(a_{11}+a_{22}\right) \tau \lambda+a_{11} a_{22} \tau^{2}-a_{12} a_{21} \tau^{2} e^{-\lambda}=0
$$

Lema 4.2 .1

(i) Se $\left|a_{11} a_{22}\right|>\left|a_{12} a_{21}\right|$ ou $a_{11} a_{22}=-a_{12} a_{21} \neq 0$, entào, temos que (4.4) nám tem raizes imaginárias, para todo $\tau>0$.

(ii) Drfinindo $\rho>0$ por:

$$
\rho=\left[\frac{1}{2}\left(-\left(a_{11}^{2}+a_{22}^{2}\right)+\sqrt{\left(a_{11}^{2}-a_{22}^{2}\right)^{2}+4 a_{12}^{2} a_{21}^{2}}\right)\right]^{1 / 2},
$$

se. $\left|a_{1} r_{22}\right|<\left|a_{12} a_{21}\right|$, entäo, para $\sigma \geq 0$, 土i $\sigma$ sào raizes de (4.4) se. c somente se, existe $n \in \mathbb{N}$ tal que $\sigma=\sigma(n), \tau=\tau(n)$, onde $\sigma(n), \tau(n)$ são definidos pelas equaçôes:

$$
\frac{\sigma(n)}{\tau(n)}=\rho \cdot \cos \sigma(n)=\frac{a_{11} a_{22}-\rho^{2}}{a_{12} a_{21}}, \operatorname{sen} \sigma(n)=\frac{\left(a_{11}+a_{22}\right) \rho}{a_{12} a_{21}}
$$


$r(n) \in(2 n \pi, 2(n+1) \pi]$

(iii) Se: $a_{11} a_{22}-a_{12} a_{21} \neq 0$, entio, temos que $\lambda=0$ f a inica miz de (4.1) rom porte real zro. para todo $\tau>0$. Temos anda, que $\lambda=0$ é uma raiz dupla de (4.4) se. e somente: si'.

$$
a_{11} a_{22}\left(a_{11}+a_{22}\right)>0
$$

r. $\tau_{0}+\mathrm{r}(\mathrm{m})$

$$
T_{0}=\frac{a_{11}+a_{22}}{a_{11} a_{22}} .
$$

(iv) $S e a_{11} a_{22}=a_{i 2}\left(l_{21}=0\right.$, entao, temos que $\lambda=0$ é a unica raiz de (4.4) com parte ral zero, para lodo $\mathrm{T}>0$. Temos ainda, $\lambda=0$ é unno raiz dupla de (4.4) se. osomente se.

$$
a_{11}=a_{22}=a_{12} a_{21}=0 \text {. }
$$

\section{Demonstração:}

Definindo $a=-\left(a_{11}+a_{22}\right), b=a_{11} a_{22}, c=-a_{12} a_{21}$, podemos reserever (4.4) como:

$$
\Delta(\lambda, \tau)=\lambda^{2}+a \tau \lambda+b \tau^{2}+c \tau^{2} e^{-\lambda} \text {. }
$$

$\Lambda$ existencia de mun real $\sigma \geq 0$ tial que $\Delta(i \sigma, \tau)=0,011$ seja.

$$
-\sigma^{2}+a \tau i \sigma+b \tau^{2}+c \tau^{2} c^{i \sigma}=0
$$

equivale à,

$$
-\sigma^{2}+b \tau^{2}+a \tau i \sigma=-c \tau^{2}(\cos \sigma-i \operatorname{sen} \sigma)
$$

donde obtemos:

$$
\left\{\begin{array}{l}
c \tau^{2} \cos \sigma=\sigma^{2}-b \tau^{2} \\
\operatorname{ctsen} \sigma=a \sigma .
\end{array}\right.
$$

Sabemos que:

$$
c^{2} \cos ^{2} \sigma+c^{2} \operatorname{sen}^{2} \sigma=c^{2},
$$

intiōo, usando o sistema acima, tenos que:

$$
\rho^{4}+\left(a^{2}-2 b\right) \rho^{2}+\left(b^{2}-c^{2}\right)=0,
$$

onde $\rho=\frac{\sigma}{r}$

Sendo assim,

$$
\rho^{2}=\frac{-\left(a_{11}^{2}+a_{22}^{2}\right)+\sqrt{\left(a_{11}^{2}-a_{22}^{2}\right)^{2}+4 a_{12}^{2} a_{21}^{2}}}{2} \geq 0 .
$$

"portanto,

$$
\sqrt{\left(a_{11}^{2}-a_{22}^{2}\right)^{2}+4 a_{12}^{2} a_{21}^{2}} \geq\left(a_{11}^{2}+a_{22}^{2}\right) \text {. }
$$

o que cquivale à:

$$
\left|a_{11} a_{22}\right| \leq\left|a_{12} a_{21}\right| .
$$


Ainda mais, $\left|a_{11} a_{22}\right|=\left|a_{12} a_{22}\right|$ se, e somente se, $\rho=0$. Conseciuentemente, temus cune se $\sigma>0$, então $\rho=\frac{\sigma}{\tau}>0$, o que implica $\left|a_{11} a_{22}\right|<\left|a_{12} a_{21}\right|$.

Obtenues de (4.9) gue $\Delta(i \sigma, \tau)=0$ se $\sigma=-\sigma(n)$ e $\tau=\tau(n)$ são como em (4.6), para alumut $n \in \mathbb{N}$.

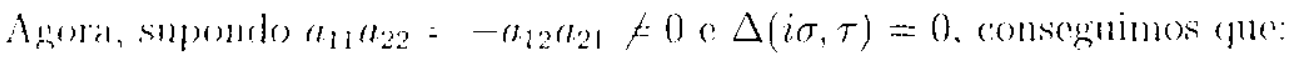

$$
\rho^{4}+\left(a^{2}-2 b\right) \rho^{2}+\left(b^{2}-c^{2}\right)=0 \Leftrightarrow \rho^{2}\left(\rho^{2}+\left(a^{2}-2 b\right)\right)=0 .
$$

(111 sejia.

$$
\left\{\begin{array}{l}
\rho^{2}=-a^{2}+2 c^{2}=-a_{11}^{2}-a_{22}^{2}<0 \\
\rho=0
\end{array}\right.
$$

Mas, como $\rho \geq 0$ obtemos $\rho=0$, o que implica $\sigma=0$.

Por ontro lado, $\Delta(0, \tau)=\tau^{2}(b+c)=2 \tau a_{13} a_{22} \neq 0$, o que contradiz nossa suposiçào.

Assim, concluńmos os ítens (i) e (ii).

Suponhamos $a_{11} a_{22}=a_{12} a_{21}$ e que existe um real $\sigma \geq 0$ tal que $\Delta(i \sigma, \tau)=0$. Analogamente ao que fizemos acima, temos de $a_{11} a_{22}=a_{12} a_{21}$ que $\left|a_{11} a_{22}\right|=\left|a_{12} a_{21}\right|$, o que refuivale à $\rho=0$, e então $\sigma=0$ e $\Delta(0, \tau)=\tau^{2}(b-\cdots b)=0$, ou seja, $\lambda=0$ é a única raì de $(4.4)$.

Observemos que:

$$
\frac{\partial \Delta}{\partial \lambda}(\lambda, \tau)=2 \lambda+a \tau-c \tau^{2} e^{-\lambda} \text { e } \frac{\partial^{2} \Delta}{\partial \lambda^{2}}(\lambda, \tau)=2+c \tau^{2} e^{-\lambda}
$$

$\log 0$.

$$
\frac{\partial \Delta}{\partial \lambda}(0, \tau)=a \tau-i \tau^{2} \text { e } \frac{\partial^{2} \Delta}{\partial \lambda^{2}}(0, \tau)=2+c \tau^{2}
$$

Suponhamos que $e=-a_{12} a_{21} \neq 0$ e $a c=\left(a_{11}+a_{22}\right) a_{12} a_{21}>0$, então $\lambda=0$ é raiz simples se, e somente se,

$$
\frac{\partial \Delta}{\partial \lambda}(0, \tau)=a \tau-c \tau^{2} \neq 0
$$

o cure be equivalente da

$$
\tau \neq \tau_{0}=\frac{a}{c}=\frac{\left(a_{11}+a_{22}\right)}{a_{12} a_{21}} .
$$

Agora, se $\tau=\tau_{0}$, temos que:

$$
\frac{\partial^{2} \Delta}{\partial \lambda^{2}}\left(0, \tau_{0}\right)=2+c \tau_{0}^{2} \neq 0
$$

o) (une equivale it

$$
a_{11}^{2}+a_{22}^{2} \neq 0
$$

e $\lambda=0$ é raiz dupla.

Por último, se $b=c=0$, então:

$$
\frac{\partial \Delta}{\partial \lambda}(0, \tau)=a \tau=0,
$$


se. e somentese,

$$
a=0
$$

Entale, para $a=b=a=0$, temos que:

$$
\frac{\partial \Delta}{\partial \lambda}(0, \tau)=0 \text { e } \frac{\partial^{2} \Delta}{\partial \lambda^{2}}(0, \tau)=2 \neq 0 .
$$

(ull sejal. $\lambda=0$ é raiz dupla de (4.4).

(om isso. conclúnuos os ítens (iii) e (iv).

Nos casos (iii) e (iv) acima, se $\lambda=0$ é um autovalor simples de (4.3), então o ponto (le equilíbrio $E^{*}$ é um ponto de sela, ver [14]. O próximo teorema apresenta as outras possíveis singularidades para o ponto de equilíbrio $E^{*}$.

\section{Teorema 4.2.1}

(i) Assumindo que $\left|a_{11} a_{22}\right|<\left|a_{12} a_{21}\right|$ e $\sigma(n), \tau(n)$ säo definidos como em (4.6), então, trmos que a bifurcaçao de Hopf ocorre sobre a variedade central para (4.1) no ponlo de runilibrio $E^{*}$ para $\tau=\tau(n)$, associada com os autovalores $\pm i \sigma(n)$ da equaçáo linearizado. $(1.3)$.

(ii) Assumindo que $a_{11} a_{22}=a_{12} a_{21} \neq 0$ e $a_{11} a_{22}\left(a_{11}+a_{22}\right)>0$, entaio, o ponto de cquihbrio $E^{*}$ de (4.1) é uma singularidade de Bogdanov-Takens para $\tau=\tau_{0}$, onde $\tau_{0} e^{\prime}$ darlo por (4.8).

(iii) Assumindo que $a_{11}=a_{22}=a_{12} a_{21}=0$ e $\left(a_{12}^{2}+a_{21}^{2}\right)>0$, então, o ponto de cquilibrio $E^{*}$ de (4.1) é uma singularidade de Bogdanov-Takens para todo $\tau>0$.

\section{Demostração:}

Assumindo que $\left|a_{11} a_{22}\right|<\left|a_{12} a_{21}\right|$ e $\lambda=\mu+i \omega$ é raiz de (4.4), isto é.

$$
\Delta(\lambda, \tau)=(\mu+i \omega)^{2}+a \tau(\mu+i \omega)+b \tau^{2}+c e^{-(\mu+i \omega)}=0
$$

onde a.b, $c$ são como no Lema 4.2.1, obtemos:

$$
\left\{\begin{array}{l}
c \tau^{2} e^{-\mu} \cos \omega=-\mu^{2}+\omega^{2}-a \tau \mu-b \tau^{2} \\
c \tau^{2} e^{-\mu \operatorname{sen} \omega}=(2 \mu+a \tau) \omega
\end{array}\right.
$$

Definimos $f_{1}, f_{2}$ por:

$$
\begin{aligned}
& f_{1}(\mu, \omega, \tau)=-\mu^{2}+\omega^{2}-a \tau \mu-b \tau^{2}-c \tau^{2} e^{-\mu} \cos \omega, \\
& f_{2}(\mu, \omega, \tau)=(2 \mu+a \tau) \omega-c \tau^{2} e^{-\mu} \operatorname{sen} \omega,
\end{aligned}
$$

e entào.

$$
\operatorname{dct}\left[D_{(h, \omega)}\left(f_{1}, f_{2}\right)(\mu, \omega, \tau)\right]=\operatorname{det}\left[\begin{array}{cc}
-2 \mu-a \tau+c \tau^{2} e^{-\mu} \cos \omega & 2 \omega+c \tau^{2} e^{-\mu} \operatorname{sen} \omega \\
2 \omega+c \tau^{2} e^{-\mu} \operatorname{sen} \omega & 2 \mu+a \tau-c \tau^{2} e^{-\mu} \cos \omega
\end{array}\right]
$$


$--\left(-2 \mu-a \tau+r \tau^{2} c " \cos \omega\right)^{2}-\left(2 \omega+c \tau^{2} e^{-\mu} \operatorname{sen} \omega\right)^{2}<0$.

Em particular, para $T(n)$ como em (4.6), temos:

$$
\operatorname{det}\left[D_{(\mu, \omega)}\left(f_{1}, f_{2}\right)(\mu, \omega, \tau(n))\right]<0,
$$

"protanto porlenos aplicar o Teorema de Funçäo Inplicita. o pual nos fornece para $\tau$ proximo a $\tau(n)$, cue existe uma curva de autovalores

$$
\lambda(\tau)=\cdots(\tau)+i \omega(\tau)
$$

$\operatorname{com} \mu(\tau(n))=0, \omega(\tau(n))>0$, ainda,

$$
\dot{\lambda}(\tau)=(\dot{\mu}(\tau), \dot{\omega}(\tau))=-\left[D_{(\mu, \omega)}\left(f_{1}, f_{2}\right)(\mu, \omega, \tau)\right]^{\cdots 1} D_{\tau}\left(f_{1}, f_{2}\right)(\mu, \omega, \tau) .
$$

Considerando

$$
\begin{aligned}
& k_{1}=-2 \mu-a \tau+c \tau^{2} e^{-\mu} \cos \omega, \\
& k_{2}=2 \omega+c \tau^{2} e^{-\mu} \operatorname{sen} \omega,
\end{aligned}
$$

whtemos que?

$$
\begin{gathered}
{\left[D_{(\mu, \omega)}\left(f_{1}, f_{2}\right)(\mu, \omega, \tau)\right]^{-1}=\left[\begin{array}{cc}
\frac{k_{1}}{k_{1}^{2}+k_{2}^{2}} & \frac{k_{2}}{k_{1}^{2}+k_{2}^{2}} \\
\frac{k_{2}}{k_{1}^{2}+k_{2}^{2}} & \frac{-k_{1}}{k_{1}^{2}+k_{2}^{2}}
\end{array}\right]} \\
D_{\tau}\left(f_{1}, f_{2}\right)(\mu, \omega, \tau)=\left[\begin{array}{c}
-a \mu-2 b \tau-2 c \tau e^{-\mu} \cos \omega \\
a \omega-2 c \tau e^{-\mu} \operatorname{sen} \omega
\end{array}\right] .
\end{gathered}
$$

Daí, conseguimos que:

$$
\begin{aligned}
& \mu(\tau(n)) \stackrel{\substack{n \cdots \omega(\tau(n)) \\
\omega \cdots \omega(\tau))}}{=} \frac{-\left(-2 \mu-a \tau(n)+c \tau^{2}(n) e^{-\mu} \cos \omega\right)\left(-a \mu-2 b \tau(n)-2 c \tau(n) e^{-\mu} \cos \omega\right)}{\left(-2 \mu-a \tau(n)+c \tau^{2}(n) e^{-\mu} \cos \omega\right)^{2}+\left(2 \omega+c \tau^{2}(n) e^{-\mu} \operatorname{sen} \omega\right)^{2}} \\
& +\frac{-\left(2 \omega+c \tau^{2}(n) e^{-\mu} \operatorname{sen} \omega\right)\left(a \omega-2 c \tau(n) e^{\mu} \operatorname{sen} \omega\right)}{\left(-2 \mu-a \tau(n)+c \tau^{2}(n) e^{-\mu} \cos \omega\right)^{2}+\left(2 \omega+c \tau^{2}(n) e^{-\mu} \operatorname{sen} \omega\right)^{2}} \\
& \stackrel{\substack{\mu(\tau(n))=0 \\
\omega(\tau(n))=\sigma(n)}}{=} \frac{-\left(-a \tau(n)+c \tau^{2}(n) \cos \sigma(n)\right)(-2 b \tau(n)-2 c \tau(n) \cos \sigma(n))}{\left(-a \tau(n)+c \tau^{2}(n) \cos \sigma(n)\right)^{2}+\left(2 \sigma(n)+c \tau^{2}(n) \operatorname{sen} \sigma(n)\right)^{2}}+ \\
& +\frac{-\left(2 \sigma(n)+c \tau^{2}(n) \operatorname{sen} \sigma(n)\right)(a \sigma(n)-2 c \tau(n) \operatorname{sen} \sigma(n))}{\left(-a \tau(n)+c \tau^{2}(n) \cos \sigma(n)\right)^{2}+\left(2 \sigma(n)+c \tau^{2}(n) \operatorname{sen} \sigma(n)\right)^{2}} \\
& (4.10) \quad \frac{-\left(-a \tau(n)-b \tau^{2}+\sigma^{2}(n)\right)\left(-2 \tau(n) \rho^{2}(n)\right)}{\left(a \tau(n)+b \tau^{2}(n)-\sigma^{2}(n)\right)^{2}+(2 \sigma(n)+a \tau(n) \sigma(n))^{2}}+ \\
& +\frac{-(2 \sigma(n)+a \tau(n) \sigma(n))(-a \sigma(n))}{\left(a \tau(n)+b \tau^{2}(n)-\sigma^{2}(n)\right)^{2}+(2 \sigma(n)+a \tau(n) \sigma(n))^{2}}
\end{aligned}
$$




$$
\begin{aligned}
= & \frac{-2 a \tau^{2}(n) \rho^{2}(n)-2 b \tau(n) \rho^{2}(n) \tau^{2}(n)+2 \tau(n) \rho^{2}(n) \sigma^{2}(n)}{\left(a \tau(n)+b \tau^{2}(n) \cdots \sigma^{2}(n)\right)^{2}+(2 \sigma(n)+a \tau(n) \sigma(n))^{2}}+ \\
& +\frac{2 a \sigma^{2}(n)+a^{2} \tau(n) \sigma^{2}(n)}{\left(n \tau(n)+b \tau^{2}(n) \cdots \sigma^{2}(n)\right)^{2}+(2 \sigma(n)+a \tau(n) \sigma(n))^{2}} \\
= & \frac{\tau(n) \sigma^{2}(n)\left(a^{2}-2\left(b-\rho^{2}(n)\right)\right)}{\left(a \tau(n)+b \tau^{2}(n)-\sigma^{2}(n)\right)^{2}+(2 \sigma(n)+a \tau(n) \sigma(n))^{2}},
\end{aligned}
$$

onder $\rho(n)=\frac{\sigma(n)}{\tau(n)}$

Cono temos $\rho$ dado por (4.5), segue que:

$$
\begin{aligned}
a^{2}-2\left(b-\rho^{2}\right) & =\left(a_{11}+a_{22}\right)^{2}-2 a_{11} a_{22}+\left(-\left(a_{11}^{2}+a_{22}^{2}\right)+\sqrt{\left(a_{11}^{2}-a_{22}^{2}\right)^{2}+4 a_{12}^{2} a_{21}^{2}}\right) \\
& =\left(a_{11}+a_{22}\right)^{2}-\left(a_{11}+a_{22}\right)^{2}+\sqrt{\left(a_{11}^{2}-a_{22}^{2}\right)^{2}+4 a_{12}^{2} a_{21}^{2}} \\
& =\sqrt{\left(a_{11}^{2}-a_{22}^{2}\right)^{2}+4 a_{12}^{2} a_{21}^{2}},
\end{aligned}
$$

Contio, $\dot{\mu}(\tau(n))>0$.

Portanto, as condliçós de Hopf são satisfeitas e a bifurcação de Hopf ocorre para $\tau=\tau(n)$.

Para tratamos os casos (ii) o (iii), consideremos o gerador infinitesimal associado ao oprerador soluçio da equação (4.3), dado por:

$$
\begin{aligned}
& A_{\tau}: D\left(A_{\tau}\right) \longrightarrow C, \\
& A_{\tau}(\phi)=\dot{\phi},
\end{aligned}
$$

onde $D\left(A_{\tau}\right)=\left\{\phi \in C^{1}: \dot{\phi}(0)=L_{\tau}(\phi)\right\}$.

Agora, suponhamos que valem as hipóteses dadas em (ii), ou sejá.

$$
a_{11} a_{22}=a_{12} a_{21} \neq 0 \text { e } a_{11}^{2} a_{22}^{2}\left(a_{11}+a_{22}\right)>0 .
$$

Provaremos que:

$$
\begin{aligned}
& \operatorname{dimKer}\left(A_{\tau}\right)=1, \operatorname{dimKer}\left(A_{\tau}^{2}\right)=1, \operatorname{para} \tau \neq \tau_{0}, \\
& \operatorname{dimKer}\left(A_{\tau_{0}}\right)=1, \operatorname{dimKer}\left(A_{\tau_{0}}^{2}\right)=\operatorname{dimKer}\left(A_{\tau_{0}}^{3}\right)=2 .
\end{aligned}
$$

Como $\lambda=0$ tem multiplicidade dois para $\tau=\tau_{0}$, pelo Lema 1.5.4, obtemos que $\operatorname{dim} \operatorname{Ker}\left(\Lambda_{\tau_{0}}^{3}\right) \leq 2$. Assim, basta nostrarmos que:

$$
\begin{aligned}
& \operatorname{dim} \operatorname{Ker}\left(A_{\tau}\right)=1, \operatorname{dim} \operatorname{Ker}\left(A_{\tau}^{2}\right)=1, \text { para } \tau \neq \tau_{0}, \\
& \operatorname{dimK\operatorname {Ker}}\left(A_{\tau_{0}}\right)=1, \operatorname{dim} \operatorname{Ker}\left(A_{\tau_{0}}^{2}\right)=2 .
\end{aligned}
$$


- Tomemos $b \in \operatorname{Ker}\left(A_{\tau}\right)$, ou soja.

$$
A_{\tau} \phi \equiv 0 \circ \dot{\phi}(0)=L_{\tau}(\phi)
$$

(miti).

$$
\begin{gathered}
\phi(\theta)=\left(\begin{array}{c}
b_{1} \\
b_{2}
\end{array}\right), \theta \in[-r, 0] \\
\left\{\begin{array}{l}
\tau a_{11} b_{1}+\tau a_{12} b_{2}=0 \\
\tau a_{21} b_{1}+\tau a_{22} b_{2}=0
\end{array}\right.
\end{gathered}
$$

Como o determinante da matriz dos coeficientes do sistema acina é zero, para todo $\tau \geq 0$. podemos tomar $b_{2}$ em função $b_{1}$,

$$
b_{2}=-\frac{a_{11}}{a_{12}} b_{1}
$$

comb beal.

Logo.

$$
\operatorname{Ker}\left(A_{\tau}\right)=\left\{\phi \in C: \phi(\theta)=\left(\begin{array}{l}
b_{1} \\
b_{2}
\end{array}\right), \operatorname{com} b_{2}=-\frac{a_{11}}{a_{12}} b_{1}\right\}
$$

Portanto, olitemos:

$$
\operatorname{dim} \operatorname{Ker}\left(A_{\tau}\right)=1
$$

Hatat todo $\tau \geq 0$.

- Consideremos $\phi \in \operatorname{Ker}\left(A_{\tau}^{2}\right)$, então:

$$
A_{\tau}^{2} \phi \equiv 0, \phi^{(2)}(0)=L_{\tau}(\dot{\phi}) \text { e } \dot{\phi}(0)=L_{\tau}(\phi) .
$$

or sijat,

$$
\begin{aligned}
& \phi(\theta)=\left(\begin{array}{l}
b_{1} \\
b_{2}
\end{array}\right)+\theta\left(\begin{array}{l}
c_{1} \\
c_{2}
\end{array}\right), \theta \in[-r, 0], \\
& \left\{\begin{array}{l}
\tau a_{11} c_{1}+\tau a_{12} c_{2}=0 \\
\tau a_{21} c_{1}+\tau a_{22} c_{2}=0,
\end{array}\right. \\
& \left\{\begin{array}{l}
\tau a_{11} b_{1}+\tau a_{12}\left(b_{2}-r_{2} c_{2}\right)=c_{1} \\
\tau a_{21}\left(b_{1}-r_{1} c_{1}\right)+\tau a_{22} b_{2}=c_{2} .
\end{array}\right.
\end{aligned}
$$

Como o determinante do sistema (4.11) é zero, para todo $\tau \geq 0$, podemos tomar $c_{2}$ an fumçào de ris.

$$
c_{2}=-\cdots \frac{a_{11}}{a_{12}} c_{1}
$$

com r real.

Multiplicando a primeira equação do sistema (4.12) por $a_{22}$, e a segunda equação por 
n1:. ohtomos:

$$
\left\{\begin{array}{l}
\tau a_{11} a_{22} b_{1}+\tau a_{12} a_{22} b_{2} \cdots \tau a_{12} a_{22} r_{2} c_{2}-a_{22} c_{1}=0 \\
\tau a_{12} a_{21} b_{1}+\tau a_{12} a_{22} b_{2}-\tau a_{12} a_{21} r_{1} a_{1}-a_{12} c_{2}=0
\end{array}\right.
$$

('omo $c_{2}=\ldots \frac{a_{11}}{a_{12}} c_{1}$ e pela hipótese $a_{11} a_{22}=a_{12} a_{22} \neq 0$, conseguimos:

$$
\left\{\begin{array}{l}
\tau a_{11}\left(a_{22} b_{1}+\tau a_{12}\left(a_{22} b_{2}+\left(\tau a_{11} a_{22}\left(1-r_{1}\right)-a_{22}\right) r_{1}=0\right.\right. \\
\tau a_{11} a_{22} b_{1}+\tau a_{12} a_{22} b_{2}+\left(-\tau a_{12} a_{22} r_{1}+a_{11}\right) c_{1}-0 .
\end{array}\right.
$$

Desse? modo,

$$
\tau a_{11} a_{22}\left(1-r_{1}\right)-a_{22}=-\tau a_{12} a_{21} r_{1}+a_{11}
$$

onlentào,

$$
\tau=\tau_{0}=\frac{\left(a_{11}+a_{22}\right)}{a_{11} a_{22}}
$$

Portanto. obtemos, que para $\tau \neq \tau_{0}$, as linhas do sistema (4.13) são linearmente independentes e portanto, subtraindo as linhas do sistema, temos:

$$
\left(\tau-\tau_{0}\right) c_{1}=0,
$$

entraro, $c_{1}=0$, consequentemente $c_{2}=0$.

Retomemos o sistemal (4.13) cons $c_{1}=c_{2}=0$,

$$
\tau\left(a_{11} a_{22} b_{1}+\tau a_{12} a_{22} b_{2}=0\right.
$$

I 0 go,

$$
b_{2}=-\frac{a_{11}}{a_{12}} b_{1} .
$$

Portanto, segue que $\operatorname{Ker}\left(A_{\tau}^{2}\right)$, para $\tau \neq \tau_{0}$, é formado pelas funções $\phi$ da forma:

$$
\phi(\theta)=\left(\begin{array}{l}
b_{1} \\
b_{2}
\end{array}\right)
$$

(am $b_{2}=-\frac{a_{11}}{a_{12}} b_{1}$

$\log 0$,

$$
\operatorname{dim} \operatorname{Ker}\left(A_{\tau}^{2}\right)=1
$$

para todo $\tau \neq \tau_{0}$.

Por outro lado, se $\tau=\tau_{0}$, as linhas do sistema (4.13) são linearmente dependentes,

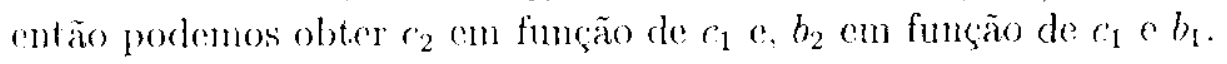

Daí, segue que,

$$
\operatorname{Kor}\left(A_{\tau_{1}}^{2}\right)=\left\{\phi \in C: \phi(\theta)=\left(\begin{array}{l}
b_{1} \\
b_{2}
\end{array}\right)+\theta\left(\begin{array}{l}
c_{1} \\
r_{2}
\end{array}\right)\right\}
$$

com

$$
c_{2}=-\frac{a_{11}}{a_{12}} c_{1} \odot b_{2}=\left(\frac{a_{11}}{a_{12}}\left(1-r_{1}\right)-\frac{1}{\tau a_{12}}\right) c_{1}-\frac{a_{11}}{a_{12}} b_{1}
$$


f'ortanto.

$$
\operatorname{dimKer}\left(A_{7_{0}}^{2}\right)=2
$$

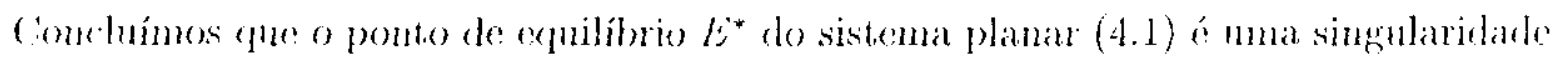
du tipo Bogdanov-Takens, para $\tau=\tau_{0}$.

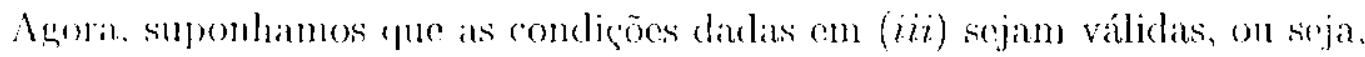

$$
a_{11}=a_{22}=a_{12} a_{21}=0 \text { e } a_{12}^{2}+a_{21}^{2}>0 \text {. }
$$

provaremos que

$$
\operatorname{dimKer}\left(A_{\tau}\right)=1, \operatorname{dimKer}\left(A_{\tau}^{2}\right)=\operatorname{dimKer}\left(A_{\tau}^{3}\right)=2, \text { para } \tau>0 .
$$

Como $\lambda=0$ tem multipliciclade dois para $\tau>0$, polo lema 1.5.4. obtemos que $\operatorname{dim} \operatorname{Ker}\left(A_{T_{0}}^{3}\right) \leq 2$. Assim, basta mostrarmos que:

$$
\operatorname{dimker}\left(A_{\tau}\right)=1, \operatorname{dimKer}\left(A_{7}^{2}\right)=2, \text { para } \tau>0 .
$$

Suponhamos sem perda de generalidade que $a_{12} \neq 0$, então, o operador linear $L_{\tau}$ ć dardo por:

$$
L_{\tau}(\phi)=\left[\begin{array}{c}
\tau a_{12} \phi_{2}\left(-r_{2}\right) \\
0
\end{array}\right]
$$

onde $\phi=\left(\begin{array}{l}\phi_{1} \\ \phi_{2}\end{array}\right) \in C$

Calcularemos os espaços $\operatorname{Ker}\left(A_{\tau}\right)$ e $\operatorname{Ker}\left(A_{\tau}^{2}\right)$.

- Tomemos $\phi \in \operatorname{Ker}\left(A_{\tau}\right)$, entäo:

$$
A_{\tau} \phi \equiv 0 \circ \dot{\phi}(0)=L_{\tau}(\phi)
$$

(n1).̇̃o.

$$
\phi(\theta)=\left(\begin{array}{l}
b_{1} \\
b_{2}
\end{array}\right), \theta \in[-r, 0]
$$

$$
\tau a_{12} b_{2}=0 \Rightarrow b_{2}=0 \text {. }
$$

Logo,

$$
\operatorname{ker}\left(A_{\tau}\right)=\left\{\phi \in C: \phi(\theta)=\left(\begin{array}{c}
b_{2} \\
0
\end{array}\right), \operatorname{com} b_{1} \in \mathbb{R}\right\}
$$

Portanto, obtenos:

$$
\operatorname{dim} \operatorname{Ker}\left(A_{\tau}\right)=1
$$

parat todo $\tau>0$

- Considerenos $\phi \in \operatorname{Ker}\left(A_{\tau}^{2}\right)$, entào

$$
A_{r}^{2} \phi \equiv 0, \phi^{(2)}(0)=L_{\tau}(\dot{\phi}) \text { e } \dot{\phi}(0)=L_{\tau}(\phi) .
$$


inl sijat,

$$
\phi(\theta)=\left(\begin{array}{l}
b_{1} \\
b_{2}
\end{array}\right)+\theta\left(\begin{array}{l}
c_{1} \\
c_{2}
\end{array}\right), \theta \in[-r, 0]
$$

('ill)

$$
\left\{\begin{array}{l}
\tau\left(u_{12} c_{2}=0\right. \\
\tau a_{12}\left(b_{2}-r_{2} c_{2}\right)=c_{1} \\
c_{2}=0
\end{array}\right.
$$

Entaio, do sistema (1.14), temos:

$$
c_{2}=0 \quad \mathrm{c} \quad b_{2}=\tau^{-1} a_{12}^{-1} c_{1}
$$

Logo, obtemos:

$$
\operatorname{ker}\left(A_{\tau}^{2}\right)=\left\{\phi \in C: \phi(\theta)=\left(\begin{array}{c}
b_{1} \\
b_{2}
\end{array}\right)+\theta\left(\begin{array}{c}
c_{1} \\
0
\end{array}\right) \operatorname{com} b_{2}=\tau^{-1} a_{12}^{-1} c_{1}\right\}
$$

para todo $\tau>0$

Portanto, temos que a dimensão do $k$ er $\left(A_{\tau}^{2}\right)$ é igual a dois, para todo $\tau>0$.

Concluínos, assin, que o ponto de opulíbrio $F^{*}$ do sistema planar (4.1) o mma singularidade do tipo Bogdanov-Takens para todo $r>0$

\subsection{Singularidade de Bogdanov-Takens}

Nesta seção, usaremos o método da forma normal para equações diferenciais funcionais retardadas com parâmetro para obtermos o fluxo do sistema planar (4.1) sobre a variedade contral bidimensional associada. Esta variedade central é tangente ao autoespaço associado com o autovalor duplo $\lambda=0$ da equação linearizada em uma vizinhlança do ponto do equilíbrio. Calcularemos também a forma normal para o modelo presa-predador.

Consideremos a equação (4.1), e suponhamos que $a_{11} a_{22}=a_{12} a_{21}$ com $a_{i j}$ dados em (4.3). Nesse caso, após a translaçào:

$$
u=y-E^{*}=\left(y_{1}-x_{1}^{*}, y_{2}-x_{2}^{*}\right)^{T},
$$

obtemos que (4.2) tem a forma:

$$
u_{u}(t)=L_{\tau}\left(u_{t}\right)+F_{\tau}\left(u_{t}\right), u_{t} \in C=C\left([-1,0], \mathbb{R}^{2}\right),
$$

onder os operadores $L_{\tau}: C \longrightarrow \mathbb{R}^{2}, F_{\tau}: C \longrightarrow \mathbb{R}^{2}$ são dados por:

$$
\begin{aligned}
& L_{\tau}(\phi)=\tau L(\phi)=\tau\left(\begin{array}{c}
a_{11} \phi_{1}(0)+a_{12} \phi_{2}\left(-r_{2}\right) \\
a_{21} \phi_{1}\left(-r_{1}\right)+a_{22} \phi_{2}(0)
\end{array}\right), \\
& F_{\tau}(\phi)=\left(\begin{array}{c}
f_{1}\left(x_{1}^{*}+\phi_{1}(0), x_{2}^{*}+\phi_{2}\left(-r_{2}\right)\right) \\
f_{2}\left(x_{1}^{*}+\phi\left(-r_{1}\right), x_{2}^{*}+\phi_{2}(0)\right)
\end{array}\right)-L_{\tau}(\phi),
\end{aligned}
$$


para $\left(\begin{array}{l}\phi_{1} \\ \phi_{2}\end{array}\right) \in C$

(omo $f_{1}, f_{2}$ sào de classe $C^{k}, k \geq 2$, porlemos definir:

$$
a_{j k}^{(i)}-\frac{\partial^{2} f_{i}\left(E^{*}\right)}{\partial x_{j} \partial x_{k}}, i=1,2, j, k=0,1,2, j+k:-2:
$$

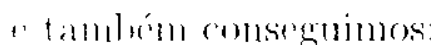

$$
u(l) \cdots L_{r}\left(u_{t}\right)+F_{2_{\tau}}\left(u_{t}\right)+I R\left(u_{t}\right),
$$

onde:

$$
F_{2 \tau}(\phi)=\tau\left(\begin{array}{c}
a_{20}^{(1)} \phi_{1}^{2}(0)+a_{11}^{(1)} \phi_{1}(0) \phi_{2}\left(-r_{2}\right)+a_{02}^{(1)} \phi_{2}^{2}\left(-r_{2}\right) \\
a_{20}^{(2)} \phi_{1}^{2}(0)+a_{11}^{(2)} \phi_{1}(0) \phi_{2}\left(-r_{2}\right)+a_{02}^{(2)} \phi_{2}^{2}\left(-r_{2}\right)
\end{array}\right) \text { e } R(\phi)=O\left(|\phi|^{3}\right) .
$$

1. Caso: $a_{11}=a_{22}=a_{12} a_{21}=0$ e $a_{12}^{2}+a_{21}^{2}>0$.

Sem perda de generalidade, considerenos $a_{12} \neq 0$. Então, o operador linear

$$
L_{T}: C \rightarrow \mathbb{R}^{2}
$$

rm (4.15) é clado por:

$$
L_{\tau}(\phi)=\left(\begin{array}{c}
\tau a_{12} \phi_{2}\left(-r_{2}\right) \\
0
\end{array}\right)
$$

Obtemos do Teorema 4.2.1, que para $\tau>0$ fixo, o ponto de equilíbrio $E^{*}$ do sistema planar (4.1) tem singularidade de Bogdanov-Takens e segue de (3.60), com $n=2$, que as respectivas bases $\Phi, \Psi$ de $P, P^{T}$ são:

$$
\begin{array}{ll}
\Phi(\theta)=\left(\phi_{1}(\theta), \phi_{2}(\theta)\right), & -1 \leq \theta \leq 0, \\
\Psi(s)=\operatorname{col}\left[\psi_{1}(s), \psi_{2}(s)\right], & 0 \leq s \leq 1,
\end{array}
$$

com

$$
\begin{aligned}
& \phi_{1}(\theta)=\left(\begin{array}{l}
a \\
b
\end{array}\right), \phi_{2}(\theta)=\left(\begin{array}{l}
c \\
d
\end{array}\right)+\theta\left(\begin{array}{l}
a \\
b
\end{array}\right), \\
& \psi_{1}(s)=\left(\begin{array}{ll}
z_{1} & z_{2}
\end{array}\right)-s\left(\begin{array}{ll}
w_{1} & w_{2}
\end{array}\right), \psi_{2}(s)=\left(\begin{array}{ll}
w_{1} & w_{2}
\end{array}\right),
\end{aligned}
$$

onde

$$
\begin{gathered}
\left(\begin{array}{l}
a \\
b
\end{array}\right),\left(\begin{array}{l}
c \\
d
\end{array}\right) \in \mathbb{R}^{2}, \\
\left(\begin{array}{ll}
z_{1} & z_{2}
\end{array}\right),\left(\begin{array}{ll}
w_{1} & w_{2}
\end{array}\right) \in \mathbb{R}^{2^{*}},
\end{gathered}
$$

Pela definiçào de $P$, obtemos que $\phi_{1}, \phi_{2}$ devem satisfazer:

$$
L_{\tau}\left(\phi_{1}\right)=\dot{\phi}_{1}(0) \text { e } I_{\tau}\left(\phi_{2}\right)=\dot{\phi}_{2}(0),
$$


(1) sijal.

$$
\left(\begin{array}{c}
\tau\left(a_{12} b\right. \\
0
\end{array}\right)=\left(\begin{array}{l}
0 \\
0
\end{array}\right) e\left(\begin{array}{c}
\tau\left(l_{12}(d+\theta b)\right. \\
0
\end{array}\right)=\left(\begin{array}{l}
a \\
b
\end{array}\right)
$$

putianto.

$$
b=0 \quad 0 \quad d=\frac{a}{\tau a_{12}} .
$$

Agora, tomando $a=1 \mathrm{c}:=0$, determinaremos $\Psi$ de modo que $(\Psi, \Phi)=I$.

Timosigne:

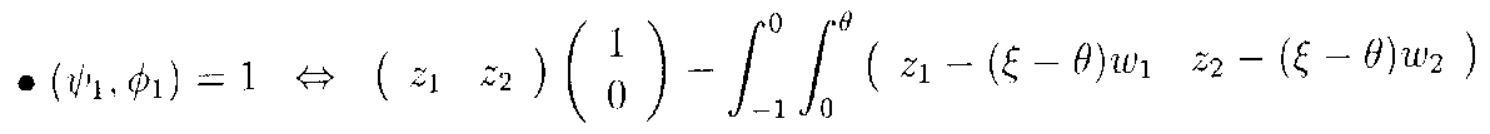

$$
\begin{aligned}
& \operatorname{d\eta }(\theta)\left(\begin{array}{c}
1 \\
0
\end{array}\right) d \xi=1 \\
& \Leftrightarrow z_{1}-\left(\begin{array}{ll}
z_{1} & z_{2}
\end{array}\right) \int_{-1}^{0} d \eta(\theta)\left(\begin{array}{l}
0 \\
0
\end{array}\right)+\left(\begin{array}{ll}
w_{1} & w_{2}
\end{array}\right) \int_{-1}^{0} d \eta(\theta)\left(\begin{array}{c}
\frac{\theta^{2}}{2} \\
0
\end{array}\right)- \\
& -\left(\begin{array}{ll}
w_{1} & w_{2}
\end{array}\right) \int_{-1}^{0} d \eta(\theta)\left(\begin{array}{c}
\theta^{2} \\
0
\end{array}\right)=1
\end{aligned}
$$

Lembrando que:

$$
\int_{1}^{0}[d \eta(\theta)] \phi(\theta)=\left(\begin{array}{c}
\tau a_{12} \phi_{2}\left(-r_{2}\right) \\
0
\end{array}\right)
$$

Concluńmos que $z_{1}=1$.

$$
\begin{aligned}
& \text { - }\left(\psi_{1}, \phi_{2}\right)=0 \Leftrightarrow\left(\begin{array}{ll}
z_{1} & z_{2}
\end{array}\right)\left(\begin{array}{c}
0 \\
\frac{1}{\tau a_{12}}
\end{array}\right)-\int_{-1}^{0} \int_{0}^{\theta}\left(\begin{array}{ll}
z_{1}-(\xi-\theta) w_{1} & \left.z_{2}-(\xi-\theta) w_{2}\right)
\end{array}\right. \\
& d \eta(\theta)\left(\begin{array}{c}
\xi \\
\frac{1}{\tau a_{12}}
\end{array}\right) d \xi=0
\end{aligned}
$$

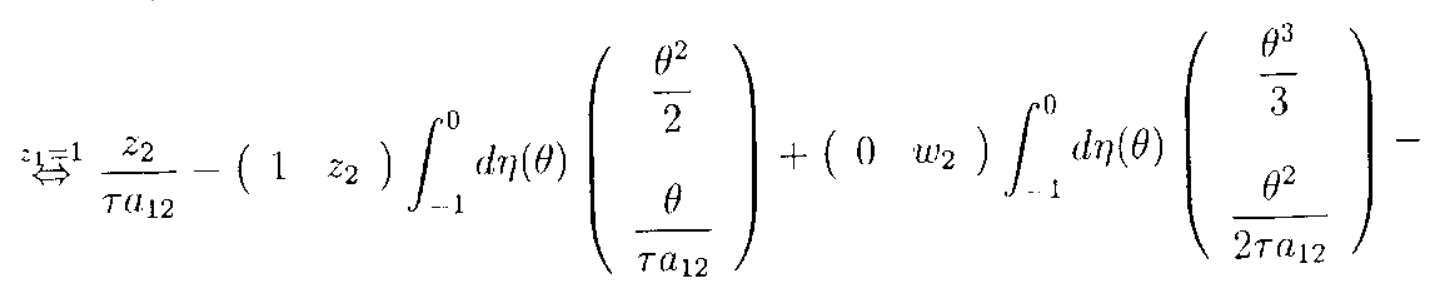

$$
\begin{aligned}
& -\left(\begin{array}{ll}
0 & z_{2}
\end{array}\right) \int_{-1}^{0} d \eta(\theta)\left(\begin{array}{c}
\frac{\theta^{3}}{2} \\
\frac{\theta^{2}}{\tau a_{12}}
\end{array}\right)=0 \text {. }
\end{aligned}
$$


Dombersegur gure

$$
z_{2}=-r_{2} r a_{12}
$$

$\bullet\left(w_{2}, w_{2}\right)-1 \Leftrightarrow\left(w_{1} \quad w_{2}\right)\left(\begin{array}{c}0 \\ \frac{1}{\tau a_{12}}\end{array}\right)-\int_{-1}^{0} \int_{0}^{\theta}\left(\begin{array}{ll}w_{1} & w_{2}\end{array}\right) d \eta(\theta)\left(\begin{array}{c}\xi \\ \frac{1}{\tau a_{12}}\end{array}\right) d \xi=1$

$$
\Leftrightarrow \frac{w_{2}}{\tau a_{12}}-\left(\begin{array}{ll}
0 & w_{2}
\end{array}\right) \int_{-1}^{0} d \eta(\theta)\left(\begin{array}{c}
\frac{\theta^{2}}{2} \\
\frac{\theta}{\tau a_{12}}
\end{array}\right)=1
$$

Portanto,

$$
w_{2}=\tau a_{12}
$$

- $\left(\psi_{2}, \phi_{1}\right)=0 \Leftrightarrow\left(\begin{array}{ll}w_{1} & w_{2}\end{array}\right)\left(\begin{array}{l}1 \\ 0\end{array}\right)-\int_{-1}^{0} \int_{0}^{\theta}\left(\begin{array}{ll}w_{1} & w_{2}\end{array}\right) d \eta(\theta)\left(\begin{array}{l}1 \\ 0\end{array}\right) d \xi=0$

$$
\Leftrightarrow w_{1}-\int_{-1}^{1)} \int_{0}^{\theta}\left(\begin{array}{ll}
w_{1} & w_{2}
\end{array}\right) d \eta(\theta)\left(\begin{array}{l}
1 \\
0
\end{array}\right) d \xi=0
$$

Logo.

$$
w_{1}=0
$$

Portanto, as bases $\Phi, \Psi$ de $P, P^{T}$, são dadas, respectivamente, por:

$$
\begin{aligned}
& \Phi(\theta)=\left(\begin{array}{cc}
1 & \theta \\
0 & \frac{1}{\tau a_{12}}
\end{array}\right), \theta \in[-1,0], \\
& \Psi(s)=\left(\begin{array}{cc}
1 & -\tau a_{12}\left(r_{2}+s\right) \\
0 & \tau a_{12}
\end{array}\right), s \in[0,1] .
\end{aligned}
$$

Assim, a matriz $B$ que satisfaz $A_{\tau} \Phi=\Phi B, A_{\tau}^{T} \Psi=B \Psi$ é dada por:

$$
B=\left[\begin{array}{ll}
0 & 1 \\
0 & 0
\end{array}\right]
$$

onde $A_{T}$ ó o gerador infinitesinal associado ao $C_{0}$-semigrupo $T_{T}(t), t \geq 0$, da equação linearizada.

Agora, introduriremos dois novos parâmetros considerando os pequenos coeficientes $a_{21}=n_{1}, a_{22}=\alpha_{2} \mathrm{~cm}(4.15)$. Daí,

$$
\dot{u}=L_{\tau}(\alpha)\left(u_{t}\right)+F\left(u_{\ell}\right), u_{t} \in C,
$$


(um $\gamma=\left(\alpha_{1}, \gamma_{2}\right) \in V \subset \mathbb{R}^{2} e L_{\tau}: V \times C \longrightarrow \mathbb{R}^{2}$ é definido por:

$$
L_{+}(\alpha)(\phi)=\left(\begin{array}{c}
a_{12} \phi_{2}\left(-r_{2}\right) \\
\alpha_{1} \phi_{1}\left(-r_{1}\right)+\alpha_{2} \phi_{2}(0)
\end{array}\right)=\cdots L_{\tau}(0)(\phi)+L_{2}(\phi)
$$

omile

$$
L_{2}(\phi)=\left(\begin{array}{c}
0 \\
\alpha_{1} \phi_{1}\left(-r_{1}\right)+\alpha_{2} \phi_{2}(0)
\end{array}\right)
$$

Assim, obtemos de (3.34) que

$$
f_{2}^{1}(x, 0, \alpha)=\Psi(0)\left[2 \tau\left(\begin{array}{c}
0 \\
\alpha_{1}\left(\Phi\left(-r_{1}\right) x\right)_{1}+\alpha_{2}(\Phi(0) x)_{2}
\end{array}\right)+F_{2_{r}}(\Phi x)\right],
$$

onde $(\Phi(\theta) x)_{1},(\Phi(\theta) x)_{2}$ sĩo as componentes da matriz, $2 \times 1, \Phi(\theta) x$, com $\theta \in[-1,0]$.

Usando a Definição 1.17 , conseguimos:

$$
\begin{aligned}
& F_{2_{+}}(\Phi x)=\tau\left(\begin{array}{c}
a_{20}^{(1)}\left[(\Phi(0) x)_{1}\right]^{2}+2 a_{11}^{(1)}(\Phi(0) x)_{1}\left(\Phi\left(-r_{2}\right) x\right)_{2}+a_{02}^{(1)}\left[\left(\Phi\left(-r_{2}\right) x\right)_{2}\right]^{2} \\
a_{20}^{(2)}\left[\left(\Phi\left(-r_{1}\right) x\right)_{1}\right]^{2}+2 a_{11}^{(2)}\left(\Phi\left(-r_{1}\right) x\right)_{1}(\Phi(0) x)_{2}+a_{02}^{(2)}\left[(\Phi(0) x)_{2}\right)^{2}
\end{array}\right) \\
& =\tau\left(\begin{array}{c}
a_{20}^{(1)} x_{1}^{2}+\frac{2 a_{11}^{(1)}}{\tau a_{12}} x_{1} x_{2}+\frac{a_{02}^{(1)}}{\left(\tau a_{12}\right)^{2}} x_{2}^{2} \\
a_{20}^{(2)} x_{1}^{2}+\left(\frac{2 a_{11}^{(2)}}{\tau a_{12}}-2 a_{20}^{(2)} r_{1}\right) x_{1} x_{2}+\left(a_{02}^{(2)} r_{1}^{2}-\frac{2 a_{11}^{(2)} r_{1}}{\tau a_{12}}+\frac{a_{02}^{(2)}}{\left(\tau a_{12}\right)^{2}}\right) x_{2}^{2}
\end{array}\right) .
\end{aligned}
$$

Daí, como

$$
\Psi(0)=\left(\begin{array}{cc}
1 & -\tau a_{12} r_{2} \\
0 & \tau a_{12}
\end{array}\right),
$$

temos que:

$$
\begin{aligned}
& f_{2}^{1}(x, 0, \alpha)=\tau\left[2\left(\begin{array}{c}
-\tau a_{12} r_{2} \\
\tau a_{12}
\end{array}\right) \alpha_{1} x_{1}+\left(\begin{array}{c}
\tau a_{12} r_{2} r_{1} \\
-\tau a_{12} r_{1}
\end{array}\right) \alpha_{1} x_{2}+\left(\begin{array}{c}
-r_{2} \\
1
\end{array}\right) \alpha_{2} x_{2}+\right. \\
& +\left(\begin{array}{c}
a_{20}^{(1)}-\tau a_{12} a_{20}^{(2)} r_{2} \\
\tau a_{12} a_{20}^{(2)}
\end{array}\right) x_{1}^{2}+\left(\begin{array}{c}
\frac{2 a_{11}^{(1)}}{\tau a_{12}}-2 r_{2} a_{11}^{(2)}+2 \tau a_{12} a_{20}^{(2)} r_{1} r_{2} \\
2 a_{11}^{(2)}-2 \tau a_{20}^{(2)} a_{12} r_{1}
\end{array}\right) x_{1} x_{2}+ \\
& \left.+\left(\begin{array}{c}
\frac{a_{20}^{(1)}}{\left(\tau a_{12}\right)^{2}}-\tau a_{12} a_{20}^{(2)} r_{1}^{2} r_{2}+2 a_{11}^{(2)} r_{1} r_{2}-\frac{a_{20}^{(2)} r_{2}}{\tau a_{12}} \\
a_{20}^{(2)} r_{1}^{2}-2 a_{11}^{(2)} r_{1}+\frac{a_{02}^{(2)}}{\tau a_{12}}
\end{array}\right) x_{2}^{2}\right]
\end{aligned}
$$




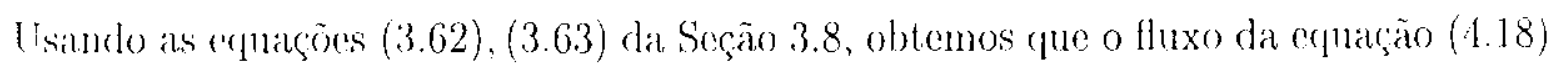
solure a variedarle contral de $E^{*}$ até os termos de segunda ordem, é dado por:

$$
\left\{\begin{array}{l}
\dot{x}_{1}=x_{2}+O\left(|(x, \alpha)|^{3}\right) \\
\dot{x}_{2}=\lambda_{1} x_{1}+\lambda_{2} x_{2}+B_{1} x_{1}^{2}+B_{2} x_{1} x_{2}+O\left(\mid(x, \theta)_{3}^{3}\right)
\end{array}\right.
$$

w1 k t

$$
B_{1}=-\frac{\tau^{2}\left(a_{12} a_{20}^{(2)}\right.}{2} . B_{2}=-\tau\left(a_{211}^{(1)} a_{11}^{(2)}\right)-\tau^{2} a_{12} a_{21)}^{(2)}
$$

- os parâmetros de bifurcação são dados por:

$$
\lambda_{1}=\tau^{2} a_{12} \alpha_{1}, \lambda_{2}=-\tau^{2} a_{12} \alpha_{1}+\tau \alpha_{2} .
$$

Podenos resumir esse caso no seguinte teorema.

Teorema 4.3.1 Suponhamos $a_{11}=a_{22}=a_{21}=0, a_{12} \neq 0$. O fluro sobre a variedade central de $F^{*}=\left(x_{1}^{*}, x_{2}^{*}\right)$ e parra todo $\tau>0$, é dedo por:

$$
\left\{\begin{array}{l}
\dot{x}_{1}=x_{2}+O\left(|x|^{3}\right) \\
\dot{x}_{2}=B_{1} x_{1}^{2}+B_{2} x_{1} x_{2}+O\left(|x|^{3}\right)
\end{array}\right.
$$

(om $B_{1}, B_{2}$ definidos por $(4.20)$. Se $B_{1} B_{2} \neq 0$, cutäo temos que o ponto de equilibrio E* i uma singularidade do tipo Bogdanov-Takens, para a qual o sistema:

$$
\left\{\begin{array}{l}
\dot{x}_{1}=f_{1}\left(x_{1}(t), x_{2}\left(t-r_{2}\right)\right) \\
\dot{x}_{2}=\alpha_{1}\left(x_{1}\left(t-r_{1}\right) \cdots x_{1}^{*}\right)+\alpha_{2}\left(x_{2}(t)-x_{2}^{*}\right)+f_{2}\left(x_{1}\left(t-r_{1}\right), x_{2}(t)\right),
\end{array}\right.
$$

6́ a versio geral. l'ara esse uiltimo sistema, a forma normal até segunda ordern sobre a waricdade central de $E^{*}$ é dada por (4.19), com $B_{1}, B_{2}$ e $\lambda_{1}, \lambda_{2}$ dados, respectiontente, $\operatorname{por}(-1.20)$ e (4.21).

Para exemplificar, descreveremos o modelo presa-predador com dois retardos.

Exemplo 4.3.1 Consideraremos o modelo presa-predador da scguinte forma:

$$
\begin{aligned}
& x_{1}(t)=x_{1}(t)\left[c_{1}-b_{1} x_{1}(t)-a \frac{x_{2}\left(t-\tau_{2}\right)}{b+x_{1}^{2}(t)}\right], \\
& x_{2}(t)=x_{2}(t)\left[-c_{2}+b_{2} \frac{x_{1}\left(t-\tau_{1}\right)}{b+x_{1}^{2}\left(t-\tau_{1}\right)}\right],
\end{aligned}
$$

crm $\tau_{1}, \tau_{2} \geq 0$ e $\tau:=\tau_{1}+\tau_{2}>0$

Supondo $b \neq 0$ e fazcndo a mudança de variácl

$$
\begin{aligned}
& x_{1} \longmapsto \frac{x_{1}}{\sqrt{b}}, \\
& x_{2} \longmapsto \frac{a x_{2}}{\sqrt{b}},
\end{aligned}
$$


bbtrmos quer a equaçào (4.23) tem a forma da equaçăo (4.1), onde:

$$
\begin{aligned}
& f_{1}\left(x_{1}, x_{2}\right)=x_{1}(t)\left[c_{1}-b_{1} x_{1}(t)-\frac{x_{2}\left(t-\tau_{2}\right)}{1+x_{1}^{2}(t)}\right] \\
& f_{2}\left(x_{1}, x_{2}\right)=x_{2}(t)\left[-c_{2}+b_{2} \frac{x_{1}\left(t-\tau_{1}\right)}{1+x_{1}^{2}\left(t-\tau_{1}\right)}\right] .
\end{aligned}
$$

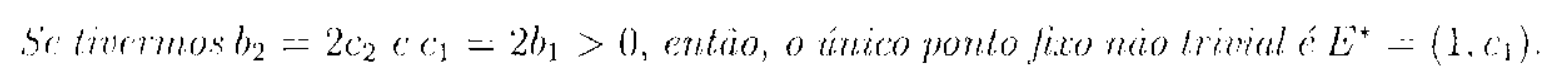
Sob essas rondiçoes. lemos:

$$
\begin{aligned}
& a_{11}=a_{21}=a_{22}=0, a_{12}=-\frac{1}{2}, \\
& a_{11}^{(1)}=a_{02}^{(1)}=0, a_{20}^{(1)}=-\frac{c_{1}}{2}, \\
& a_{11}^{(2)}=a_{02}^{(2)}=0, a_{20}^{(2)}=-c_{1} c_{2},
\end{aligned}
$$

Assim. fazendo as mudanças de variáveis:

$$
\begin{aligned}
& t \longmapsto \frac{t}{\tau} \\
& \left(x_{1}, x_{2}\right) \rightarrow\left(x_{1}-1, x_{2}-c_{2}\right),
\end{aligned}
$$

obtemos que a forma normal até ordem dois para o fluxo sobre a variedade central de $E^{*}$ i dada por (4.22) com $B_{1}, B_{2}$ como em (4.20), ou seja,

$$
B_{\mathrm{L}}=\frac{\tau^{2} c_{1} c_{2}}{8}, B_{2}=-\frac{\tau c_{1}\left(1+\tau c_{2}\right)}{2} .
$$

Agora, consideremos o sistema:

$$
\begin{aligned}
& \dot{x}_{1}(t)=x_{1}(t)\left[c_{1}-\frac{c_{1}}{2} x_{1}(t)-\frac{x_{2}\left(t-\tau_{2}\right)}{1+x_{1}^{2}(t)}\right], \\
& \dot{x}_{2}(t)=x_{2}(t)\left[-c_{2}+\beta_{1}+\beta_{2} x_{1}\left(t-\tau_{1}\right)+2 c_{2} \frac{x_{1}\left(t-\tau_{1}\right)}{1+x_{1}^{2}\left(t-\tau_{1}\right)}\right],
\end{aligned}
$$

milio:

$$
a_{21}=c_{1} \beta_{2} \quad \text { e } a_{22}=\beta_{1}+\beta_{2}
$$

logo, faźndo as mudanças de varianeis (4.24), temos a equaçâo (4.18), com

$$
\alpha_{1}=c_{1} \beta_{2}, \alpha_{2}=\beta_{1}+\beta_{2}
$$

' portanto, a forma normal até ordem dois sobre a varicdade central de $E^{*}$, é dada por (4.19), com $\lambda_{1}, \lambda_{2}$ dados por (4.21), ou seja,

$$
\lambda_{1}=-\frac{\tau^{2} c_{1} \beta_{2}}{2}, \lambda_{2}=\frac{\tau^{2} c_{1} \beta_{2}}{2}+\tau\left(\beta_{1}+\beta_{2}\right) .
$$


2" Caso: $a_{11} a_{22}=\ldots a_{12} a_{21} \neq 0 u^{\prime} a_{11} a_{22}\left(a_{11}+a_{22}\right)>0$.

Visse caso. nsando o Teorema 4.2.1, obtenos (jue para $\tau_{0}$ como em (4.8), o ponto de: "quilíbro $F^{*}$ (a cenaçào (4.17) tem singularidade de. Bogdanov-Takens.

Nesise caso temos que o operador $L: C \rightarrow \mathbb{R}^{2}$ ó dado por:

$$
L_{\tau_{0}}(\phi)=\int_{-1}^{0} d \eta(\theta) \phi(\theta)=\left(\begin{array}{c}
\tau_{0} a_{11} \phi_{1}(0)+\tau_{0} a_{12} \phi_{2}\left(-r_{2}\right) \\
\tau_{0} a_{21} \phi_{1}\left(-r_{1}\right)+\tau_{0} a_{22} \phi_{2}(0)
\end{array}\right) .
$$

onde a matriz $\eta(\theta)$ o dada por:

$$
\eta(\theta)=\left[\begin{array}{cc}
\eta_{1}(\theta) & \eta_{2}(\theta) \\
\eta_{3}(\theta) & \eta_{4}(\theta)
\end{array}\right]
$$

com

$$
\begin{aligned}
& \eta_{1}(\theta)=\left\{\begin{array}{ll}
0 & \text { se } \theta=0, \\
\tau_{0} a_{11} & \text { se } \theta \in[-1,0),
\end{array} \quad \eta_{2}(\theta)= \begin{cases}0 & \text { se } \theta \in\left[-r_{2}, 0\right], \\
\tau_{0} a_{12} & \text { se } \theta \in\left[-1,-r_{2}\right),\end{cases} \right. \\
& \eta_{3}(\theta)=\left\{\begin{array}{ll}
0 & \text { se } \theta \in\left[-r_{1}, 0\right], \\
\tau_{0} a_{21} & \text { se } \theta \in\left[-1,-r_{1}\right),
\end{array} \quad \eta_{4}(\theta)= \begin{cases}0 & \text { se } \theta=0, \\
\tau_{0} a_{22} & \text { se } \theta \in[-1,0) .\end{cases} \right.
\end{aligned}
$$

Ohtemos de (3.60), com $n=2$, que as respectivas bases $\Phi, \Psi$ de $P, P^{T}$ são:

$$
\begin{array}{ll}
\Phi(\theta)=\left(\phi_{1}(\theta), \phi_{2}(\theta)\right), & -1 \leq \theta \leq 0, \\
\Psi(s)=c o l\left[\psi_{1}(s), \psi_{2}(s)\right], & 0 \leq s \leq 1,
\end{array}
$$

(')ili

$$
\begin{aligned}
& \phi_{1}(\theta)=\left(\begin{array}{l}
a \\
b
\end{array}\right), \phi_{2}(\theta)=\left(\begin{array}{l}
c \\
d
\end{array}\right)+\theta\left(\begin{array}{l}
a \\
b
\end{array}\right), \\
& \psi_{1}(s)=\left(\begin{array}{ll}
z_{1} & z_{2}
\end{array}\right)-s\left(\begin{array}{ll}
w_{1} & w_{2}
\end{array}\right), \psi_{2}(s)=\left(\begin{array}{ll}
w_{1} & w_{2}
\end{array}\right),
\end{aligned}
$$

onde

$$
\begin{gathered}
\left(\begin{array}{c}
a \\
b
\end{array}\right),\left(\begin{array}{c}
c \\
d
\end{array}\right) \in \mathbb{R}^{2}, \\
\left(\begin{array}{ll}
u_{1} & w_{2}
\end{array}\right),\left(\begin{array}{ll}
z_{1} & z_{2}
\end{array}\right) \in \mathbb{R}^{2^{*}} .
\end{gathered}
$$

Pola definicano de $P$, obtemos que $\phi_{1}, \phi_{2}$ devem satisfazer:

$$
L_{\tau}\left(\phi_{1}\right)=\dot{\phi}_{1}(0), L_{\tau}\left(\phi_{2}\right)=\dot{\phi}_{2}(0),
$$

(3) seja,

$$
\left(\begin{array}{c}
\tau_{0} a_{11} a+\tau_{0} a_{12} b \\
\tau_{0} a_{21} a+\tau_{0} a_{22} b
\end{array}\right)=\left(\begin{array}{c}
0 \\
0
\end{array}\right),\left(\begin{array}{c}
\tau_{0} a_{11} c+\tau_{0} a_{12} d-\tau_{0} a_{12} a_{11} r_{2} \\
\tau_{11} a_{21} c+\tau_{0} a_{22} d-\tau_{0} a_{21} a_{12} r_{1}
\end{array}\right)=\left(\begin{array}{l}
a \\
b
\end{array}\right) .
$$


Como o determinante do primeiro sistema acinda é zero, segue que existem infinitas solucioss das quats podemos tomar:

$$
a=-a_{12}, b=a_{11},
$$

substituindo no segundo sistema, consegumos:

$$
c:=-a_{12} \text { e } d=a_{11}\left(1+r_{2}\right)-\tau_{0}{ }^{1},
$$

portanto, mna base $\Phi$ é dada por:

$$
\Phi(\theta)=\left(\begin{array}{cc}
-a_{12} & -a_{12}(1+\theta) \\
a_{11} & a_{11} \theta+\left(1+r_{2}\right) a_{11}-\tau_{0}^{-1}
\end{array}\right) .
$$

Da definição de $P^{T}$, obtenos que $\psi_{1}, \psi_{2}$ devem satisfazcr:

$$
\int_{-1}^{0} \psi_{1}(-\theta) d \eta(\theta)=-\dot{\psi}_{1}(0), \int_{-1}^{0} \psi_{2}(-\theta) d \eta(\theta)=-\dot{\psi}_{2}(0)=0,
$$

e nusando (4.25), conseguinos da segunda condição acima que:

$$
\begin{aligned}
& \int_{-1}^{0}\left(w_{1} d \eta_{1}(\theta)+w_{2} d \eta_{3}(\theta), w_{1} d \eta_{2}(\theta)+w_{2} d \eta_{4}(\theta)\right)=(0,0) \\
& \Leftrightarrow\left(\tau_{0} a_{11} w_{1}+\tau_{0} a_{21} w_{2}, \tau_{0} a_{12} w_{1}+\tau_{0} a_{22} w_{2}\right)=(0,0) \\
& \Leftrightarrow\left\{\begin{array}{l}
a_{11} w_{1}+a_{21} u u_{2}=0 \\
a_{12} w_{1}+a_{22} w_{2}=0
\end{array}\right.
\end{aligned}
$$

da primeira exuação, temos due:

$$
\begin{aligned}
& \int_{-1}^{0}\left(\left(z_{1}+\theta w_{1}\right) d \eta_{1}(\theta)+\left(z_{2}+\theta w_{2}\right) d \eta_{3}(\theta),\left(z_{1}+\theta w_{1}\right) d \eta_{2}(\theta)+\left(z_{2}+\theta w_{2}\right) d \eta_{4}(\theta)\right) \\
& =\left(w_{1}, w_{2}\right) \\
& \Leftrightarrow\left\{\begin{array}{l}
z_{1} \tau_{0} a_{11}+\left(z_{2}-r_{2} w_{2}\right) \tau_{y} a_{21}=w_{1} \\
\left(z_{1}-r_{2} w_{1}\right) \tau a_{12}+z_{2} \tau_{0} a_{22}=w_{2},
\end{array}\right. \\
& \Leftrightarrow\left\{\begin{array}{l}
\tau_{0} a_{11} z_{1}+\tau_{0} a_{21} z_{2}=w_{1}+\tau_{0} a_{21} r_{1} w_{2} \\
\tau a_{12} z_{1}+\tau_{0} a_{22} z_{2}=w_{2}+\tau_{0} a_{12} r_{2} w_{1},
\end{array}\right. \\
& \Leftrightarrow\left\{\begin{array}{l}
\tau_{0} a_{11} a_{12} z_{1}+\tau_{0} a_{21} a_{12} z_{2}=w_{1} a_{12}+\tau_{0} a_{21} a_{12} r_{1} w_{2} \\
\tau a_{12} a_{11} z_{1}+\tau_{0} a_{22} a_{11} z_{2}=w_{2} a_{11}+\tau_{0} a_{12} a_{11} r_{2} w_{1},
\end{array}\right.
\end{aligned}
$$




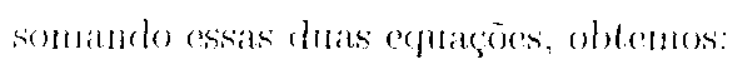

$$
2 \tau_{0} a_{11}\left(a_{12} z_{1}+a_{22} z_{2}\right)-\tau_{0} a_{12} a_{21} r_{1} w_{2}+a_{32} w_{1}+a_{11} w_{2}+\tau_{0} a_{11} a_{12} r_{2} u_{1}
$$

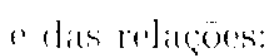

$$
w_{2}=-\frac{a_{12}}{a_{22}} w_{1} \cdot \tau_{0}=\frac{a_{11}+a_{22}}{a_{1} a_{22}} \cdot r_{2}=\left(1-r_{1}\right)
$$

Sh'Lillo (j110:

$$
2 \tau_{0} a_{11}\left(a_{12} z_{1}+a_{22} z_{2}\right)=-2 \tau_{0} a_{11} a_{12} r_{1} w_{1}+\frac{a_{11}+a_{22}}{a_{11} a_{22}} a_{11} a_{12} w_{1}+a_{12} w_{1}-\frac{a_{11} a_{12}}{a_{22}} w_{11} .
$$

portantos.

$$
2 \tau_{0} a_{11}\left(a_{12} z_{1}+a_{22} z_{2}\right)=2 a_{12}\left(1-\tau_{0} r_{1} a_{11}\right) u_{1} .
$$

Assim, os vetores $z=\left(z_{1}, z_{2}\right), w=\left(w_{1}, w_{2}\right) \in \mathbb{R}^{2^{*}}$ da base I devem satisfazer:

$$
\begin{aligned}
& a_{12} w_{1}+a_{22} u_{2}=0, \\
& \tau_{0} a_{11}\left(a_{12} z_{1}+a_{22} z_{2}\right)=a_{12}\left(1-\tau_{0} r_{1} a_{11}\right) w_{1} .
\end{aligned}
$$

Para que possamos ter $(\Psi, \Phi)=1$, duas condiçoes adicionais devem ser satisfoitas:

$$
\left(\psi_{1}, \phi_{1}\right)=1 \text { e }\left(\psi_{1}: \phi_{2}\right)=0
$$

(nil sejal,

- $\left(\psi_{1}, \phi_{1}\right)=1 \quad \Leftrightarrow \quad z \phi_{1}(0)-\int_{-1}^{0} \int_{0}^{\theta}(z-(\xi-\theta) w) d \eta(\theta) \phi_{1}(\xi) d \xi=1$

$$
\begin{aligned}
& z \phi_{1}(0)-z \int_{-1}^{0} \int_{0}^{\theta} d \eta(\theta) \phi_{1}(\xi) d \xi+w \int_{-1}^{\theta} \int_{0}^{\theta} d \eta(\theta) \xi \phi_{1}(\xi) d \xi- \\
& -w \int_{-1}^{0} \int_{0}^{\theta} d \eta(\theta) \theta \phi_{1}(\xi) d \xi=1 \\
\Leftrightarrow & z\left[\phi_{1}(0)-L_{\tau_{0}}\left(\phi_{1}(0) \theta\right)\right]-w L_{\tau_{0}}\left(\frac{\phi_{1}(0) \theta^{2}}{2}\right)=1 \\
\Leftrightarrow \quad & z L_{\tau_{0}}\left(\phi_{2}(0)\right)-w L_{\tau_{0}}\left(\frac{\phi_{1}(0) \theta^{2}}{2}\right)=1
\end{aligned}
$$

- $\left(\psi_{1}, \phi_{2}\right)=0 \quad \Leftrightarrow \quad z \phi_{2}(0)-\int_{-1}^{0} \int_{0}^{\theta} \psi_{1}(\xi-\theta) d \eta(\theta) \phi_{2}(\xi) d \xi=0$

$\Leftrightarrow \quad z\left[\phi_{2}(0)-L_{\tau_{0}}\left(\phi_{2}(0) \theta\right)-L_{\tau_{0}}\left(\phi_{1}(0) \theta^{2}\right)\right]+w\left[L_{\tau_{0}}\left(\phi_{2}(0) \theta\right)+\right.$

$$
\left.+L_{\tau_{0}}\left(\phi_{1}(0) \theta^{2}\right)-L_{\tau_{0}}\left(\phi_{2}(0) \theta^{2}\right)-L_{\tau_{0}}\left(\phi_{1}(0) \frac{\theta^{3}}{2}\right)\right]=0 \text {. }
$$


Comchindo, temos gue $(\Psi, \Phi)=I$ se:

$$
\begin{aligned}
& z L_{\tau_{0}}\left(\phi_{2}(0)\right)-w L_{\tau_{0}}\left(\frac{\phi_{1}(0) \theta^{2}}{2}\right)=1 \\
& z\left[\phi_{2}(0)-L_{\tau_{0}}\left(\phi_{2}(0) \theta\right)-L_{\tau_{0}}\left(\phi_{1}(0) \theta^{2}\right)\right]+w\left[L_{\tau_{13}}\left(\phi_{2}(0) \theta\right)+\right. \\
& +L_{\tau_{0}}\left(\phi_{1}(0)\left(\theta^{2}\right)-L_{\tau_{0}}\left(\phi_{2}(0) \theta^{2}\right)-L_{\tau_{0}}\left(\phi_{1}(0) \frac{\theta^{3}}{2}\right)\right]=0 .
\end{aligned}
$$

Observemos que as condições (4.26), (4.27), determinam univocamente un par de ve$\operatorname{tores} z, w \in \mathbb{R}^{2 *}$.

Para $a_{11} a_{22}=a_{12} a_{21}$, a bifurcaçono de Bogdanov-Takens ocorre quando $\tau$ passa por $\tau_{0}$. Consequientemente, é conveniente introduzirmos $\tau$ como un parànetro de bifurcaçäo. Paua descrevermos completamente a bifurcação, precisaremos introdurir um segundo parametro, o qual faremos fixando très dos coeficientes $a_{i j}, i, j=1,2$, e deixarenos o ontro variar. Portanto, introduzimos o parâmetro de bifurcaçăo $\alpha=-\left(\alpha_{1}, \alpha_{2}\right) \in \mathbb{R}^{2}$ recs(revendo:

$$
\begin{gathered}
\tau=\tau_{0}+\alpha_{1}, \\
a_{21}=\frac{a_{11} a_{22}}{a_{12}}+\alpha_{2} .
\end{gathered}
$$

Assim, introduzindo o parâmetro $\alpha=\left(\alpha_{1}, \alpha_{2}\right)$ em $(4.15)$, obtemos:

$$
\dot{u}(l)=L_{\tau_{0}}\left(u_{t}\right) \dashv L_{2_{\tau_{1}}}(\alpha)\left(u_{t}\right)+\tilde{F}\left(u_{t}, \alpha\right),
$$

onde.

$$
\begin{aligned}
& L_{2_{\tau_{0}}}(\alpha)(\phi)=\alpha_{1} L(\phi)+\tau_{0} \alpha_{2}\left(\begin{array}{c}
0 \\
\phi_{1}\left(-r_{1}\right)
\end{array}\right) \\
& \ddot{F}(\phi, \alpha)=\alpha_{1} \alpha_{2}\left(\begin{array}{c}
0 \\
\phi_{1}\left(-r_{1}\right)
\end{array}\right)+\left(\tau_{0}+\alpha_{1}\right) F(\phi),
\end{aligned}
$$

parat $\phi=\left(\begin{array}{c}\phi_{1} \\ \phi_{2}\end{array}\right) \in C$.

Obtemos da Seção 3.8, que:

$$
f_{2}^{1}(x, 0, \alpha)=\psi(0)\left[2 L_{2_{\tau_{0}}}(\alpha)(\Phi x)+\tau_{0} \alpha_{2} F_{2_{\tau_{0}}}(\Phi x)\right]
$$

com $F_{2_{\tau_{0}}}(\Phi x)$ dado como em (4.17), a matriz $\Psi(0)=\left(\begin{array}{cc}z_{1} & z_{2} \\ w_{1} & w_{2}\end{array}\right)$ é determinada por $(4.26),(4.27), \mathrm{e}$

$$
\begin{aligned}
L_{2 \tau_{0}}(\alpha)(\Phi, x) & =\alpha_{1}\left(\begin{array}{c}
a_{11}(\Phi(0))_{1}+a_{12}\left(\Phi\left(-r_{2}\right) x\right)_{2} \\
a_{11} a_{22} \alpha_{12}^{-1}\left(\Phi\left(-r_{1}\right) x\right)_{1}+a_{22}(\Phi(0) x)_{2}
\end{array}\right)+\tau_{0} \alpha_{2}\left(\begin{array}{c}
0 \\
\left(\Phi\left(-r_{1}\right) x\right)_{1}
\end{array}\right) \\
& =\alpha_{1}\left(\begin{array}{c}
-a_{12} \tau_{0}^{-1} x_{2} \\
0 \\
a_{22}\left(a_{11}-\tau_{0}^{-1}\right) x_{2}
\end{array}\right)+\tau_{0} \alpha_{2}\left(\begin{array}{c}
0 \\
-a_{12}\left(x_{1}+r_{2} x_{2}\right)
\end{array}\right) .
\end{aligned}
$$


Portanto, conseguimos de (3.62), (3.63) e (4.26) que a forma nomal sobre a variedarle rentral é dada por:

$$
\left\{\begin{array}{l}
\dot{x}_{1}=x_{2}+O\left(|(x, n)|^{3}\right) \\
\dot{x}_{2}=\lambda_{1} x_{1}+\lambda_{2} x_{2}+B_{1} x_{1}^{2}+B_{2} x_{1} x_{2}+O\left(|(x, \alpha)|^{3}\right)
\end{array}\right.
$$

(m)lar

$$
\begin{aligned}
B_{1}= & \frac{\tau_{0}}{2}\left[a_{12}^{2}\left(a_{20}^{(1)} w_{1}+a_{20}^{(2)} w_{2}\right)-2 a_{11} a_{12}\left(a_{11}^{(1)} w_{1}+a_{11}^{(2)} w_{2}\right)+a_{11}^{2}\left(a_{02}^{(2)} u_{1}+a_{02}^{(2)}\right)\right] \\
B_{2}= & \tau_{0}\left\{a_{12}^{2}\left(a_{20}^{(1)} z_{1}+a_{20}^{(2)} z_{2}\right)-2 a_{12} a_{11}\left(a_{11}^{(1)} z_{1}+a_{11}^{(2)} z_{2}\right)+a_{11}^{2}\left(a_{02}^{(1)} z_{1}+a_{02}^{(2)} z_{2}\right)+\right. \\
& +a_{12}^{2}\left(a_{20}^{(1)} w_{1}+r_{2} a_{20}^{(2)} w_{2}\right)-a_{12}\left[\left(2 a_{11}-\tau_{0}^{1}\right) a_{11}^{(1)} w_{1}+\left(a_{11}\left(1+2 r_{2}\right)-\right.\right. \\
& \left.\left.\left.-\tau_{0}^{-1}\right) a_{11}^{(2)} w_{2}\right]+a_{11}\left[\left(a_{11}-\tau_{0}^{1}\right) a_{02}^{(1)} w_{1}+\left(a_{11}\left(1+r_{2}\right)-\tau_{0}^{-1}\right) a_{02}^{(2)} w_{2}\right]\right\}
\end{aligned}
$$

e paranetros

$$
\begin{aligned}
& \lambda_{1}=-\tau_{0} \alpha_{12} \alpha_{2} w_{2} \\
& \lambda_{2}=-a_{12} \tau_{0} \alpha_{2}\left(z_{2}+r_{2} w_{2}\right)+a_{22} a_{11} \alpha_{1} w_{2}
\end{aligned}
$$

Podemos resumir esse caso no seguinte tcorema.

Teorema 4.3.2 Considerando os coefirientes $a_{i j}, i, j=1,2$, com om (4.3), assumimos que $a_{i . j} \neq 0$ e $a_{11} a_{22}\left(a_{11}+a_{22}\right)>0$. Sejam $\alpha_{1}, \alpha_{2}$ definidos por (4.28), e $\tau_{0}$ como $\mathrm{cm}$ (4.8). Entâo, para $\alpha_{1}=\alpha_{2}=0$, o ponto de equilíbrio $E^{*}$ da Equação (4.1) tem uma singularidade do tipo Bogdanon-Takens e a equação diferencial ordinária sobre a variedade centrol para (4.1) de $E^{*}$ é dada por (4.31), com $B_{1}, B_{2}, \lambda_{1}, \lambda_{2}$ definidos, respectivamente. $\operatorname{por}(4.32),(4.33)$.

Agora faremos um exemplo do caso acima.

Exemplo 4.3.2 Consideremos o sistema planar com dois retardos $\tau_{1}, \tau_{2}$ da forma:

$$
\begin{aligned}
& \dot{x}_{1}(t)=x_{1}(t)+g_{1}\left(x_{2}\left(t-\tau_{2}\right)\right), \\
& \dot{x}_{2}(t)=x_{2}(t)+g_{2}\left(x_{1}\left(t-\tau_{1}\right)\right),
\end{aligned}
$$

onde $g_{1}, g_{2}: \mathbb{R} \rightarrow \mathbb{R}$ säo funçôes de classe $C^{2}$, tais que:

$$
g_{1}(0)=g_{2}(0)=0, g_{1}^{\prime}(0) g_{2}^{\prime}(0) \neq 0
$$

Fasendo a mudança de variável

$$
x_{1} \longmapsto \frac{x_{1}}{g_{1}^{\prime}(0)},
$$

podemos considerar $g_{1}^{\prime}(0)=1$. É claro que $E^{*}=(0,0)$ é um ponto de equilibrio de (4.34). 
Definimalo

$$
\begin{aligned}
& f_{1}\left(x_{1}, x_{2}\right) \ldots x_{1}+y_{1}\left(x_{2}\right), \\
& f_{2}\left(x_{1}, x_{2}\right)=x_{2}+y_{2}\left(x_{1}\right),
\end{aligned}
$$

whthos

$$
\begin{aligned}
& a_{11}=a_{12}=a_{22}=1, \\
& u_{21}=y_{2}^{\prime}(0) .
\end{aligned}
$$

Supondo $g_{2}^{\prime}(0)=1$, segue que $a_{11} a_{22}=a_{12} a_{21} \neq 0 \Leftrightarrow a_{11} a_{22}\left(a_{11}+a_{22}\right)>0$. Entrio. temos que (4.34) tem singularidade do tipo Bogdanov- Takens para

$$
\tau_{i 1}=\frac{a_{11}+a_{22}}{a_{11} a_{22}}=2
$$

Para essar situaçuo, os roeficientes $a_{j k}^{(i)}$ em (4.16) sào:

$$
\begin{aligned}
& a_{20}^{(1)}=a_{11}^{(1)}=0, a_{02}^{(1)}=g_{1}^{\prime \prime}(0), \\
& a_{20}^{(2)}=g_{2}^{\prime \prime}(0), a_{11}^{(2)}=a_{02}^{(2)}-\therefore 0,
\end{aligned}
$$

r a.s condiciess em (4.26) sio dadas por:

$$
\begin{aligned}
& w_{1}+w_{2}=0, \\
& 2\left(z_{1}+z_{2}\right)=\left(1-2 r_{1}\right) w_{1},
\end{aligned}
$$

$r(o m) r_{i}=\frac{\tau_{i}}{\tau_{0}}, i=1,2$.

Sob a suposiçäo de $g_{2}^{\prime}(0)=1$, conscgumos do Teorema 4.3.2, que a forma normal sobme a variedade central da origem para $\tau_{0}=2$, até termos de ordem dois é dada por:

$$
\left\{\begin{array}{l}
\dot{x}_{1}=x_{2}+O\left(|x|^{3}\right) \\
\dot{x}_{2}=B_{1} x_{1}^{2}+B_{2} x_{1} x_{2}+O\left(|x|^{3}\right)
\end{array}\right.
$$

e os coeficientes $B_{1}, B_{2}$ em $(4.33)$, saio:

$$
\begin{aligned}
& B_{1}=\left(g_{1}^{\prime \prime}(0)-g_{2}^{\prime \prime}(0)\right) w_{1}, \\
& B_{2}=\left(g_{1}^{\prime \prime}(0)-g_{2}^{\prime \prime}(0)\right)\left(2 z_{1}+w_{1}\right),
\end{aligned}
$$

$\because, w_{1}$ saio determinados por $(4.26)$ e (4.27).

Uma nersào geral da singularidade do tipo Bogdanow-Takens, provem de (4.34), introdusindo os partimetros $\alpha_{1}=\tau-2, \alpha_{2}=g_{2}^{\prime}(0)-1$.

$$
\left\{\begin{array}{l}
\dot{x}_{1}=x_{2}+O\left((|\lambda|+|x|)|x|^{2}\right\rangle \\
\dot{x}_{2}=\lambda_{1} x_{1}+\lambda_{2} x_{2}+a x_{1}^{2}+b x_{1} x_{2}+O\left((|\lambda|+|x|)|x|^{2}\right),
\end{array}\right.
$$




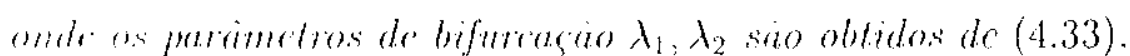

$$
\begin{aligned}
& \lambda_{1}=2 \alpha_{2} w_{1} \\
& \lambda_{2}=2 \alpha_{2} z_{1} 1+\left(\alpha_{2}-\alpha_{1}\right) w_{1} .
\end{aligned}
$$




\section{Referências Bibliográficas}

[1] BACHMAN, G.; NARICI, L. Functional Analysis. New York, 1966.

[2] Bogdanov, R. I. Versal Deformations of a Singular Point on a Plane in the case of Zero Eigenvalues 1975, 144-145; 10 (1976), 61-62.

[3] BOGDANOV, R. I. Orbital Equivalence of Singular Points of the Vector Fields on the Plane 10(1976), 316-317.

[4] CHOW, S. N.; HALE, J. K. Methods of Bifurcation Theory New York, 1996, Springer

[5] COTLAR, M.; CIGNOLI, R. An Introduction to Functional Analysis New York, 1974.

[6] HALE, J. K. Theory of Functional Differential Equations New York, 1977.

[7] HALE, J. K.; LUNEL, S. M. V. Introduction to Functional Differential Equations Applied Matt. Sciences 99, Springer-Verlag.

[8] HALE, J. K.; TANAKA, S. M. Square and Pulse Waves with Two delays J. Dynam. Diff. Eq. 10 (2000), 1-30.

[9] KUZNETSOV, Y. A. Elements of Applied Bifurcation Theory New York, 1998.

[10] TAKENS, F. Singularities of Vector Fields Publ. Math. Inst. Haustes Etudes sci 43(1974), 47-100.

[11] TAYLOR, A. Introduction to Functional Analysis New York. John \& Sons

[12] TEREZA, F. Normal Forms for Retarded Functional Differential Equations and Applications to Bogdanov-Takens, J. Differential Equations 122 (1995), 201-224.

[13] TEREZA, F. Normal Forms for Retarded Functional Differential Equations with Pararreters and Applications to Hopf Bifurcation, J. Differential Equations 122 (13:95), 181-200.

[14] TEREZA, F. On the Study of Singularities for a Planar System with Two Delays, J. Dyflamics of Continuous, Discrete and Impulsive Systems 10 (2003), 357-371.

[15] WIGGins, S. Introduction to Applied Nonlinear Dynamical Systems and Chaos New York, 1990, Springer-Verlag. 
[16] XIAO, D.; RUAN, S. Multiple Bifurcations in a Delayed Predator-Prey System with Nonmonotonic Functional Response J. Differential Equations, 176 (20)1), 494-510.

[17] ZEII)LER, E. Applied Functional Analysis: Applications to Mathematical Physics Springer-Verlag, New York, 1995. 